\title{
Election Results Common Data Format Specification
}

Version 1.0

\author{
John P. Wack \\ Kim Brace \\ Sam Dana \\ Herb Deutsch \\ John Dziurlaj \\ Ian Piper \\ Don Rehill \\ Richard Rivello \\ Sarah Whitt
}




\title{
Election Results Common Data Format Specification
}

\section{Version 1.0}

\author{
John P. Wack \\ Richard Rivello \\ Software and Systems Division \\ Information Technology Laboratory \\ Kim Brace, EDS \\ Sam Dana, Prometheus Computing \\ Herb Deutsch, ES\&S \\ John Dziurlaj, Ohio SoS Office \\ Ian Piper, Dominion Voting \\ Don Rehill, AP \\ Sarah Whitt, Wisconsin GAB
}

This publication is available free of charge from:

http://dx.doi.org/10.6028/NIST.SP.1500-100

February 2016

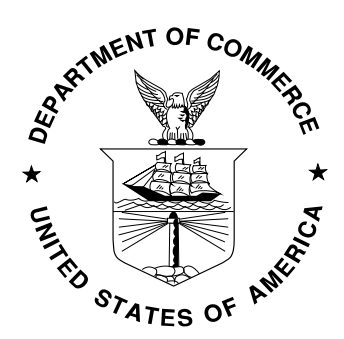

U. S. Department of Commerce Penny Pritzker, Secretary

National Institute of Standards and Technology Willie May, Under Secretary of Commerce for Standards and Technology and Director 


\section{National Institute of Standards and Technology (NIST) Special Publication 1500-100 145 pages (February 2016)}

NIST Special Publication series 1500 is intended to capture external perspectives related to NIST standards, measurement, and testing-related efforts. These external perspectives can come from industry, academia, government, and others. These reports are intended to document external perspectives and do not necessarily represent official NIST positions.

Certain commercial entities, equipment, or materials may be identified in this document in order to describe an experimental procedure or concept adequately. Such identification is not intended to imply recommendation or endorsement by NIST, nor is it intended to imply that the entities, materials, or equipment are necessarily the best available for the purpose.

There may be references in this publication to other publications currently under development by NIST in accordance with its assigned statutory responsibilities. The information in this publication, including concepts and methodologies, may be used by federal agencies even before the completion of such companion publications. Thus, until each publication is completed, current requirements, guidelines, and procedures, where they exist, remain operative. For planning and transition purposes, federal agencies may wish to closely follow the development of these new publications by NIST.

Organizations are encouraged to review all draft publications during public comment periods and provide feedback to NIST. All NIST publications are available at http://www.nist.gov/publication-portal.cfm.

National Institute of Standards and Technology

Attn: Software and Systems Division, Information Technology Laboratory 100 Bureau Drive (Mail Stop 8970) Gaithersburg, MD 20899-8930

Email: voting@nist.gov 


\title{
Reports on Computer Systems Technology
}

The Information Technology Laboratory (ITL) at the National Institute of Standards and Technology (NIST) promotes the U.S. economy and public welfare by providing technical leadership for the Nation's measurement and standards infrastructure. ITL develops tests, test methods, reference data, proof of concept implementations, and technical analyses to advance the development and productive use of information technology. This document reports on ITL's research, guidance, and outreach efforts in Information Technology and its collaborative activities with industry, government, and academic organizations.

\begin{abstract}
This publication describes an election results common data format specification for pre-election setup information and post-election results reporting. It contains a UML model of the election data and an XML format derived from the UML model. It also contains background information regarding how geopolitical geography is structured and used in the model and schema. The XML format is comprehensive and at the same time very flexible, able to accommodate election scenarios used throughout the U.S. It is part of a series of planned common data format specifications for voting equipment.
\end{abstract}

\section{Keywords}

Ballot; common data format; contest; district; election results; jurisdiction; overvote; political office; political party; precinct; referendum; undervote, voting. 


\section{Acknowledgements}

The authors wish to thank their colleagues of the National Institute of Standards and Technology VVSG-Interoperability Public Working Group, who reviewed drafts of this document and contributed to its technical content. The authors gratefully acknowledge and appreciate the following contributors for their keen and insightful assistance with developing this specification:

\begin{tabular}{|c|c|c|}
\hline $\begin{array}{l}\text { Kenneth Bennett } \\
\text { Office of Registrar-Recorder } \\
\text { /County Clerk, Los Angeles }\end{array}$ & $\begin{array}{l}\text { Lauren Massa-Lochridge } \\
\text { Independent Researcher }\end{array}$ & $\begin{array}{l}\text { Benjamin Rice } \\
\text { Dominion Voting }\end{array}$ \\
\hline Thomas Connolly & Neal McBurnett & John Sebes \\
\hline New York State Board of Elections & ElectionAudits & OSET Foundation \\
\hline Art Greisser & John McCarthy & Paul Stenbjorn \\
\hline Prometheus Computing & Verified Voting & Election Information Services \\
\hline Chris Jerdonek & Janet Modrow & Beth Ann Surber \\
\hline $\begin{array}{l}\text { Elections Commission, City and } \\
\text { County of San Francisco }\end{array}$ & Florida Division of Elections & $\begin{array}{l}\text { Office of the Secretary of State, } \\
\text { West Virginia }\end{array}$ \\
\hline Arthur Keller & Justin Moore & David Tarrent \\
\hline University of California & Google & Bureau of Elections, Michigan \\
\hline Jared Marcotte & Tammy Patrick & David Webber \\
\hline The Turnout & Bipartisan Policy Institute & Horizon Industries \\
\hline
\end{tabular}


Executive Summary

This publication is a specification for a common data format (CDF) for pre-election setup information and post-election results reporting. The format, known as the Election Results Common Data Format Specification, is comprehensive and detailed in its coverage of election results-related data and at the same time very flexible, able to accommodate election scenarios used throughout the United States. This publication contains a UML (Unified Modeling Language) model, a derived XML (eXtensible Markup Language) schema, usage information and guidance, and background information.

This specification provides a common data interchange format for election data used in voting systems across U.S. jurisdictions. Using this specification, pre-election and post-election data can be published in a common, well-understood format. The format accommodates highly detailed election results data and is sufficiently flexible to accommodate many different types of contests and political structures.

This specification provides manufacturers of election management systems (EMS) and managers of election jurisdictions with standard methods for importing and exporting election data, thereby increasing interoperability among election devices and reducing the need to create software to translate between proprietary formats. Interoperable data will reduce costs to election jurisdictions by reducing the complexity in election management and offering jurisdictions more choice in election equipment.

This specification is geared towards the following audiences:

- Election officials,

- Voting equipment manufacturers,

- Election-affiliated organizations, and

- Election analysts and the general public.

The format accommodates three different election scenarios:

Pre-election. The period prior to an election, for reporting pre-election data from a jurisdiction but not yet complete information about any election.

Election. The period during which an election is being conducted and election results reports are produced. The reports include aggregated results data or more detailed, precinct-level reporting, depending on the capabilities of the reporting jurisdiction.

Post-election. The period after the close of polls when more detailed election results reports are produced with options for precinct reporting, type of ballot, and type of device.

The XML schema associated with this specification is derived from a UML model that defines the types, structure, and interrelationships of geopolitical geography across the U.S. The model was designed to accommodate multiple types of contests and their many variations, and to provide the capability to report on these contests from higher aggregate levels down to very fine 
levels of detail, including:

- Reporting by precinct and splits of precincts;

- Reporting by ballot type, e.g., absentee, election day; and

- Reporting by device type and specific voting device.

The UML model can be re-used and modified to meet the needs of other planned common data format specifications for voting devices such as electronic pollbooks and ballot marking devices. 


\section{Table of Contents}

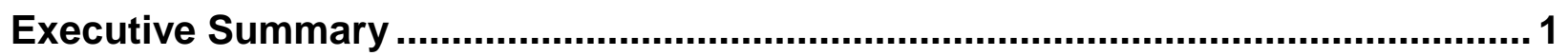

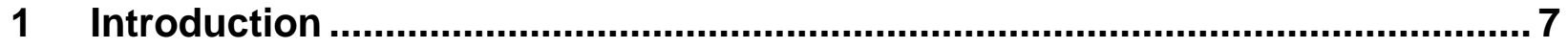

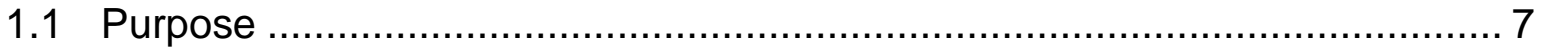

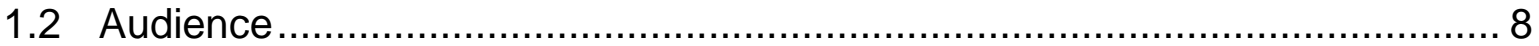

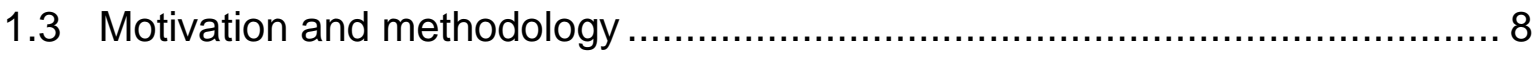

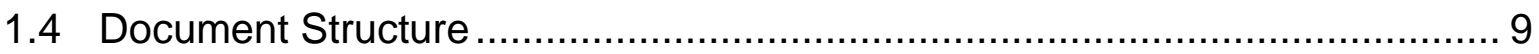

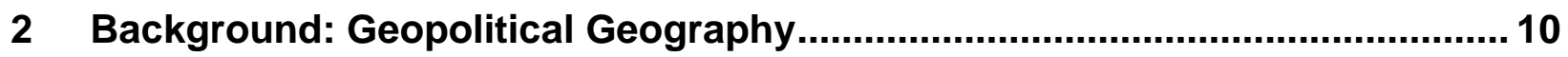

2.1 The Primary Types of Geopolitical Geography ...................................... 10

2.1.1 Governmental-based Geography …............................................ 10

2.1.2 Political-based Geography .................................................... 12

2.1.3 Administrative-based Geography …......................................... 12

2.2 Linking the Geopolitical Geographies Together ...................................... 13

2.3 Geopolitical Geography in the UML Model and XML Schema .................... 17

3 Use Cases and UML Model ......................................................................... 19

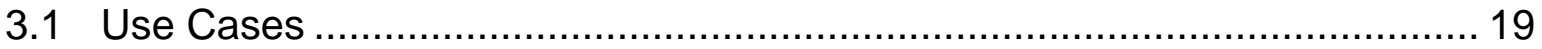

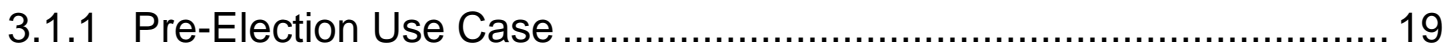

3.1.2 Election Night Reporting Use Case ............................................ 20

3.1.3 Post-Election Reporting Use Case …........................................... 21

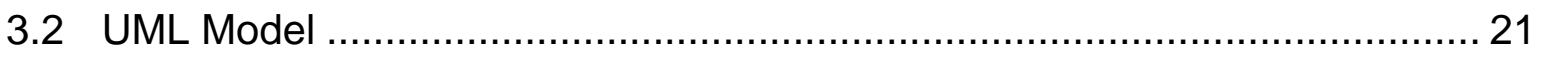

3.2.1 Major Classes..................................................................... 22

3.2.2 Relationships between Classes............................................... 24

$4 \quad$ XML Schema

4.1 Schema Stylistic Conventions.............................................................. 26

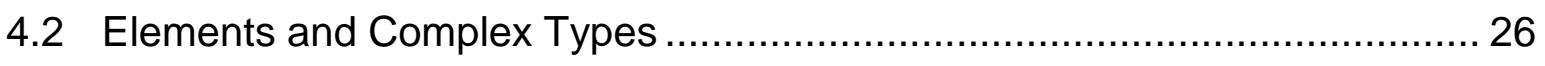

4.2.1 The <AnnotatedString> Complex Type …................................... 27

4.2.2 The <BallotSelection> Element and Extension Base....................... 28

4.2.3 The <BallotStyle> and <OrderedContest> Elements........................ 34

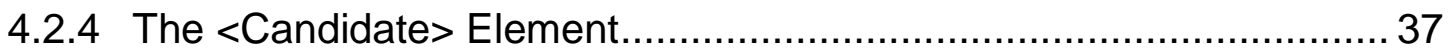

4.2.5 The <ContactInformation> Element.............................................. 39

4.2.6 The <Contest> Element and Extension Base .................................. 41 


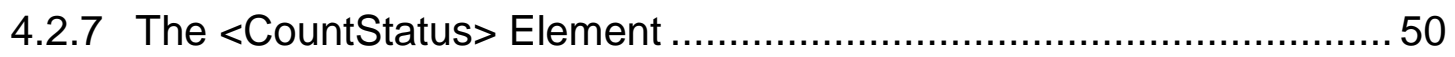

4.2.8 The <Counts> Extension Base Element.......................................... 51

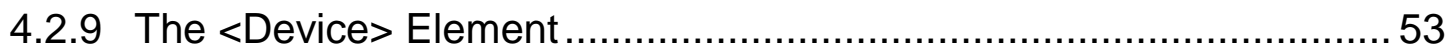

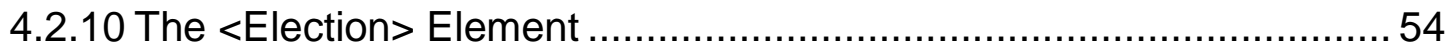

4.2.11 The <ElectionAdministration> Element.......................................... 56

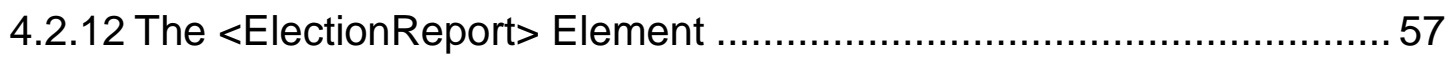

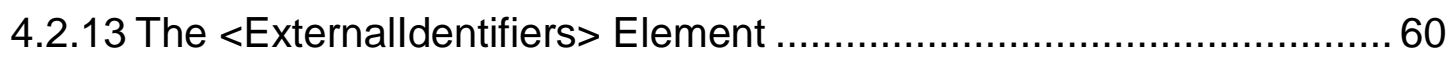

4.2.14 The <GpUnit> Element and Extension Base ................................... 62

4.2.15 The <InternationalizedText $>$ Element and <LanguageString $>$ Complex

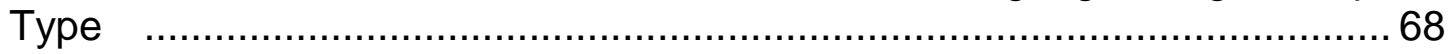

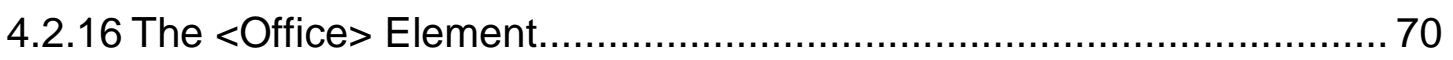

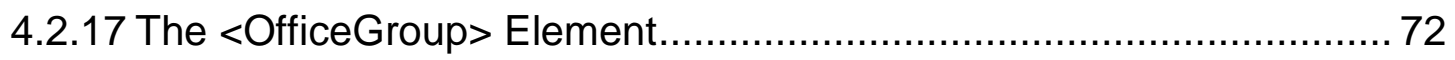

4.2.18 The <Party> Element and Extension Base ..................................... 73

4.2.19 The <PartyRegistration> Element ……….................................. 76

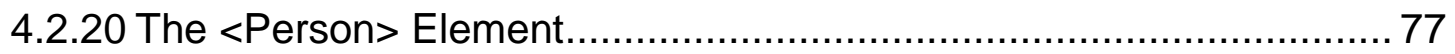

4.2.21 The <Schedule> and <Hours> Elements...................................... 79

4.2.22 The <SpatialDimension> and <SpatialExtent> Elements .................. 81

4.2.23 The <SummaryCounts> (extension base <Counts>) Element ........... 83

4.2.24 The <VoteCounts $>$ (extension base <Counts>) Element .................. 84

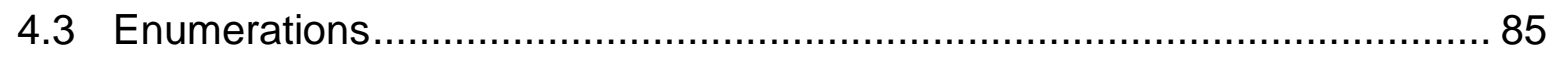

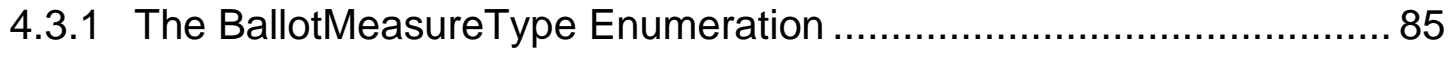

4.3.2 The CandidatePostElectionStatus Enumeration.............................. 86

4.3.3 The CandidatePreElectionStatus Enumeration ................................. 87

4.3.4 The CountltemStatus Enumeration ............................................. 88

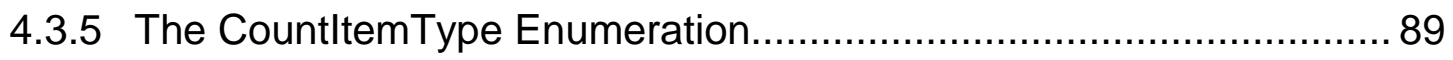

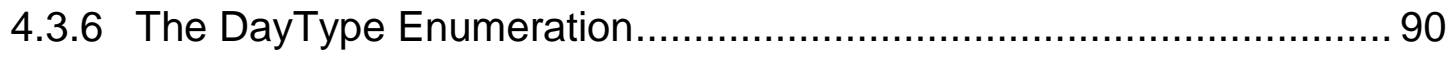

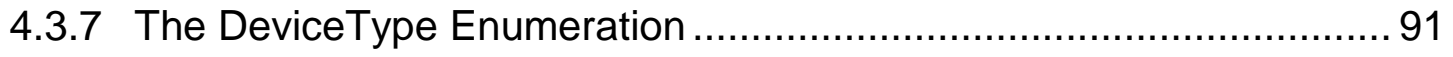

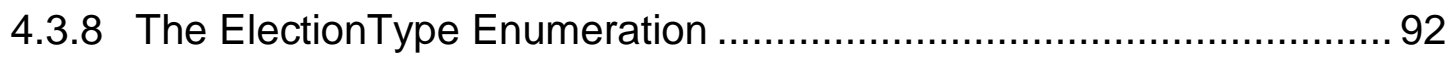

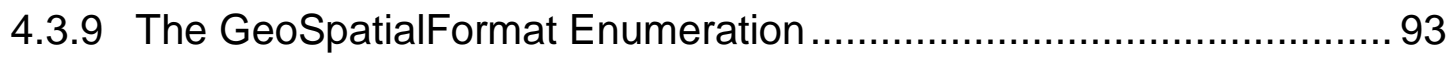

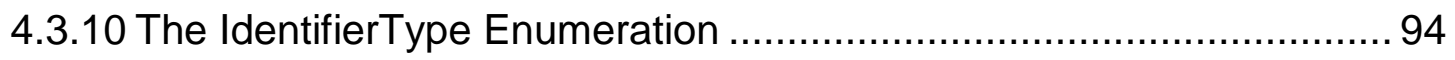

4.3.11 The OfficeTermType Enumeration ................................................... 95

4.3.12 The ReportDetailLevel Enumeration................................................ 96 
4.3.13 The ReportingUnitType Enumeration ............................................. 97

4.3.14 The ResultsStatus Enumeration ................................................... 99

4.3.15 The VoteVariation Enumeration ................................................. 100

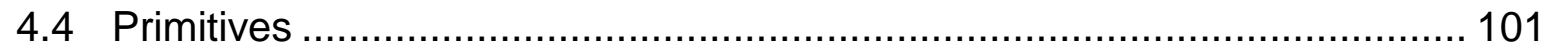

4.4.1 The HTMLColorString Primitive ................................................. 101

4.4.2 The Primitive ShortString Primitive …........................................... 101

4.4.3 The Primitive TimeWithZone Primitive.......................................... 101

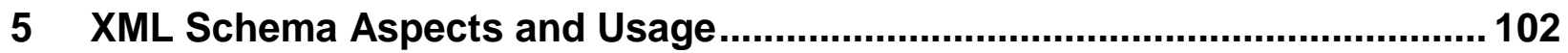

5.1 General Layout of XML Instance Files .............................................. 102

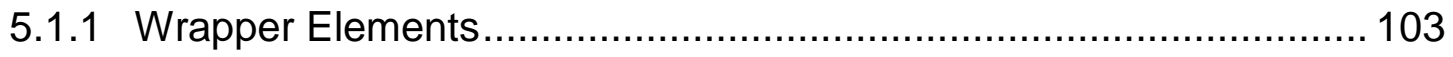

5.1.2 How UML Relationships Are Implemented in the XML Schema....... 103

5.1.3 Directed Association Relationships .............................................. 104

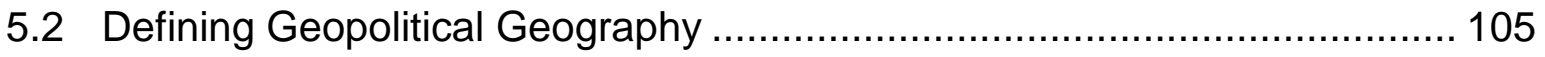

5.2.1 Defining <GpUnit> Elements for Governmental and Administrativebased Geographies..................................................................... 106

5.2.2 Associating an Election Authority with the $<$ GpUnit> Element Defined

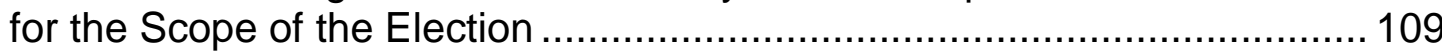

5.2.3 Defining <GpUnit> Elements for Political-based Geographies ......... 110

5.2.4 Dealing with Duplicate Electoral Districts ..................................... 111

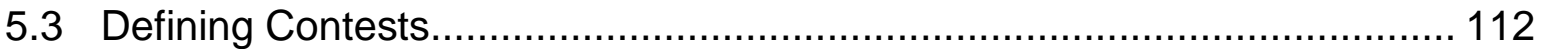

5.4 Associating Vote Counts with Contests ............................................. 114

5.4.1 Aggregated Vote Counts ........................................................ 114

5.4.2 Vote Counts by Precinct, Ballot, and Device Type ......................... 115

5.4.3 Summary Vote and Ballot Counts.............................................. 117

5.5 Associating Summary Ballot Counts with Geopolitical Units.................... 117

\section{List of Appendices}

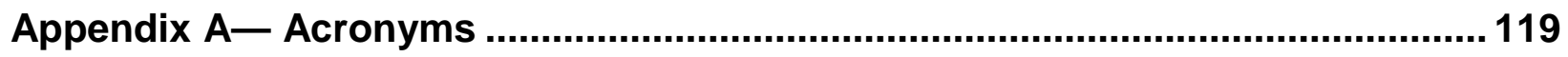

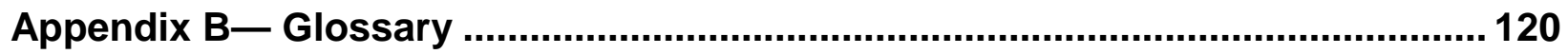

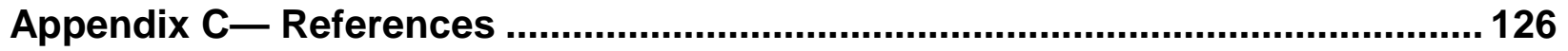

Appendix D— File Download Locations......................................................... 128

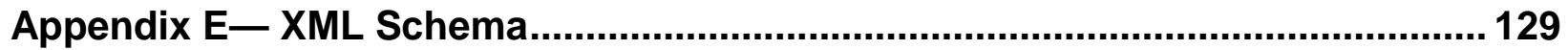

Appendix F- UML Class Diagram - Detailed..................................................... 139 


\section{List of Figures}

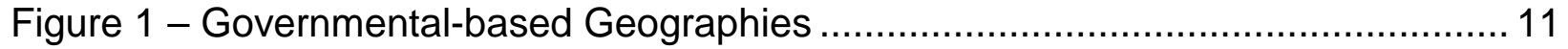

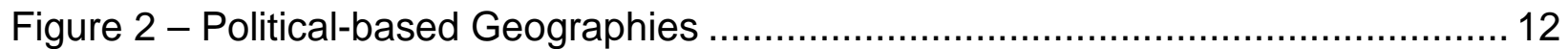

Figure 3 - Administrative-based Geographies .................................................... 13

Figure 4 - Ward and Precincts in Cambridge, MA. ............................................. 14

Figure 5 - Districts Overlaying Wards and Precincts in Cambridge, MA..................... 15

Figure 6 - Overlapping Non-hierarchical Electoral Districts ..................................... 16

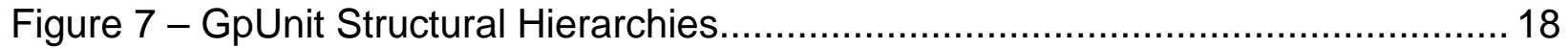

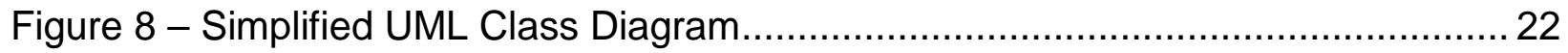

Figure 9 - Directed Composition Example ........................................................... 24

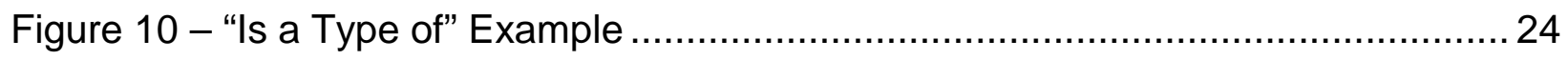

Figure 11 - Directed Association Example.................................................................. 24

Figure 12 - General Layout of Instance Files.................................................... 102

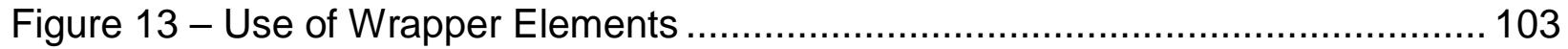

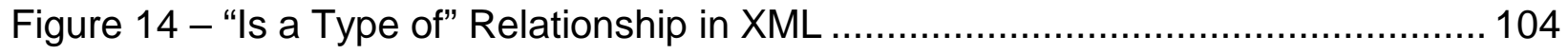

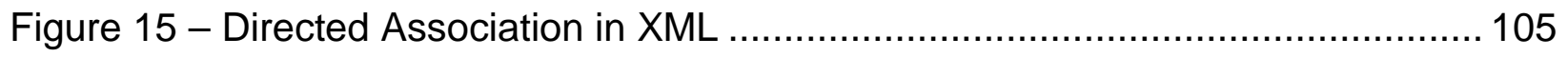

Figure 16 - Geopolitical Geography Example from Wisconsin .............................. 107

Figure $17-<$ GpUnit> Definitions for Governmental and Administrative Geographies 108

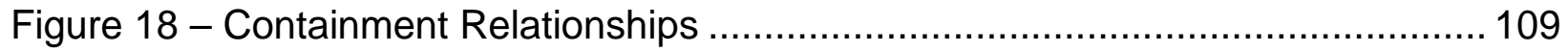

Figure 19 - Associating Election Authority Information .......................................... 109

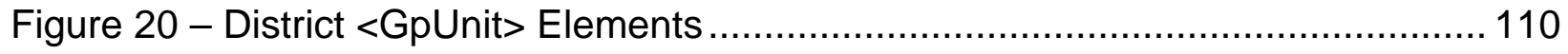

Figure 21 - Defining Political Geographies and Linking to Precincts ........................ 111

Figure 22 - Re-Using Electoral District Links ................................................... 112

Figure 23 - Basic Contest Definition and Linkage to Vote Counts ............................ 114

Figure 24 - Linking Aggregated Vote Counts to Contests........................................ 115

Figure 25 - Linking Vote Counts to Precincts ..................................................... 116

Figure 26 - Filtering Vote Counts by Device and Ballot Type ................................. 116

Figure 27 - Associating Summary Counts with Contests...................................... 117

Figure 28 - Associating Summary Ballot Counts with <GpUnit> Elements................ 118 


\section{Introduction}

This publication is a specification for an XML-based (eXtensible Markup Language) [1] common data format (CDF) for exchanging pre-election and post-election data from voting systems used for managing elections and tabulating election results across states and territories of the United States. The format serves as a basic export of election information from an election management system (EMS) and as a means for combining election data from different EMSs or transferring election data between EMSs. It defines common exchange methods between distributed voting places and central offices as well as from election offices to news media and the general public. These common exchange methods promote interoperability and eliminate the need for proprietary formats.

This specification includes a data model in UML (Unified Modeling Language) [2] that specifies and defines the data involved in pre-election setup and post-election results reporting. The XML format is derived from the UML model.

The primary features of this specification are:

- Major data elements and their attributes and associations are fully defined in a UML data model;

- The data model can be used to generate data formats (e.g., XML, JSON (JavaScript Object Notation)) for today's election systems as well as for future election systems;

- Election data and results can be reported at flexible levels from highly aggregated to very detailed;

- Detailed reporting includes by device type, by type of ballot, and by geopolitical geographies including precinct and split precinct;

- Geopolitical units of geography can be specified in a flexible manner to mirror reporting structures used across states and counties and cities;

- Major elements such as contests, geopolitical units, and parties include the capability to support multiple types of identifiers and cross-references; and

- Detailed instructions for implementation and use of the XML schema are included.

\subsection{Purpose}

The purpose of this specification is to provide a comprehensive, flexible, and interoperable preelection setup and post-election results reporting XML format for manufacturers to integrate into their voting equipment and for election offices, the media, and other groups to use in their own software. The advantages of using this specification include:

- Election results can be reported directly from election offices in this format regardless of voting system manufacturer, thus enabling interoperability;

- The need for custom software and custom reporting formats is greatly reduced;

- Jurisdictions that use multiple versions of EMSs and tabulators can more easily combine and transfer information between systems; and 
- Consistency in election results reports across different voting systems, jurisdictions, and states, will make reporting on election performance, e.g., for the EAC (Election Assistance Commission) election administration and voting survey (EAVS) [3] and other election analyses, easier and more accurate.

\subsection{Audience}

The intended audience of this specification includes election officials, manufacturers and developers, as well as others in the election community including the general public. Election results reporting is deceptively complex, thus some background in election administration or technology is useful in understanding the material in this specification.

\subsection{Motivation and methodology}

This specification was motivated primarily to reduce the inherent complexity for U.S. election officials in collecting and publishing election data, especially on election night when time frames are tight and there are more opportunities for error and a greater need for automation. The process of reporting election results is a highly complicated activity that occurs over several different time frames and in multiple scenarios. The equipment involved and data produced often do not interoperate, adding more complexity to the process. Additionally, there are sometimes significant variations among different jurisdictions within a state as well among the states themselves in the way they perform election results reporting.

NIST and a community of U.S. election officials, analysts, and voting system manufacturers investigated reporting scenarios and their associated geopolitical geographies throughout the United States and in existing and emerging voting systems. Further study included evaluation of other XML schemas associated with U.S. elections, including:

- $\quad$ The State of Florida XML schema for election results reporting [4],

- The Pew Voting Information Project XML schema version 3.0 [5],

- The OASIS Election Markup Language (EML) XML schema version 7.0 [6], and

- Schemas created by the Associated Press ${ }^{1}$.

From this analysis, three use cases were developed for election results reporting:

1. Pre-election - election data that is known ahead of the election; basically an export from an EMS of the contests, candidates, ballot initiatives, information about offices, and the geopolitical geographies associated with the reporting jurisdiction;

2. Election night - reporting of election results either summarized by contest and jurisdiction or broken down by individual reporting units such as precincts, and

\footnotetext{
${ }^{1}$ The Associated Press does not make these schemas available to the public.
} 
associated formats, either as updates or corrections to previous reports or as internal intermediate reports within a state or county; and

3. Post-election - updates and the final results compiled during the post-election canvass.

A UML data model was subsequently generated to define the data associated with the use cases and to show the relationship and organization of the data elements. Finally, an XML schema was generated from the UML data model. The XML schema defines the rules of the XML format.

The advantages of using a UML data model as an intermediate step to generating an XML schema include (1) that the model is independent of the concrete XML format or other potential formats that could be derived and (2) relationships between data elements are easier to correctly define and visualize when they are independent of any specific data format. If changes are needed to the XML format, one can make changes to the UML model and generate a new version of the format.

Note that this specification addresses U.S. governmental elections and is not intended for use "as is" in other types of elections or in other countries. However, the specification was written with the intention that it be adaptable to other election environments.

\subsection{Document Structure}

Section 2 starts with an overview of geopolitical geographies such as counties, districts, and precincts, describing how they are categorized, how they interrelate, and how election results are tied to them. Section 3 contains an overview of the three use cases for election results reporting and the UML data model that implements the use cases. Section 4 contains documentation for the XML schema that is derived from the UML model. Section 5 describes how to use the major features of the schema.

The appendices include references, definitions, acronyms, and instructions for downloading the files associated with this specification. 


\section{Background: Geopolitical Geography}

This section provides an overview of the geopolitical geography in the United States as it relates to elections and election results reporting, and serves to provide background for how geopolitical geography is implemented in the UML model and XML schema that are described in sections 3 through 5. Knowledge of what constitutes geopolitical geography and how it is interrelated and used in elections provides the underpinning for understanding the complexities of election results reporting.

\subsection{The Primary Types of Geopolitical Geography}

The primary types of geopolitical geography include those geographies that run elections such as states, counties, and cities, as well as the many types of electoral districts that are tied to contests, precincts, and various other geographical units associated with political boundaries. Generally, the media and election analysts wish to obtain voting results broken out by these units, thus the process of running an election includes associating contests and vote counts with these units so that they can be ultimately reported.

Ballot counts and vote counts for contests can be associated with a variety of different types of geopolitical geography, ranging from aggregated counts associated with a county or state down to more detailed counts associated with a precinct and breakdowns of a precinct. Precincts are generally the smallest unit of geopolitical geography, and in many states, there is generally one polling place per precinct. Precincts can be thought of as the bricks or building blocks that compose all other geopolitical geography.

Geopolitical geography can often be quite complex in that some are hierarchical, others overlap, and still others change their boundaries regularly, sometimes several times within a year. Changes to city and district boundaries affect precinct boundaries, splitting them into multiple parts (called split precincts), with each part requiring distinct ballot styles.

The following sections break down geopolitical geography into three primary types and show how the geographies interrelate. These three types are:

1. Governmental-based geography,

2. Political-based geography, and

3. Administrative-based geography.

\subsubsection{Governmental-based Geography}

Governmental-based geography refers to entities that (1) run elections and (2) are wellestablished and do not change over time, with the exception of some cities. For many states, the governmental-based geography is hierarchical, as shown in Figure 1. This can be categorized as follows: 
- States,

- Counties,

- Cities,

- American Indian Reservations,

- Towns and Townships, and

- Other Civil Divisions.

Nearly all states have counties, although some use different words to describe them, e.g., parishes for Louisiana and boroughs for Alaska. Townships occur in 20 states and adhere to county boundaries. In the six New England states, townships run the election process and there is no county government, thus election results are reported directly to the state. Municipalities (cities, towns, or villages) in Michigan, Minnesota, and Wisconsin also run their elections, but report their information to the county, which then reports to the state. Other civil divisions include boroughs as used in Connecticut, New Jersey, Pennsylvania, and other states; New York City's boroughs are treated as counties.

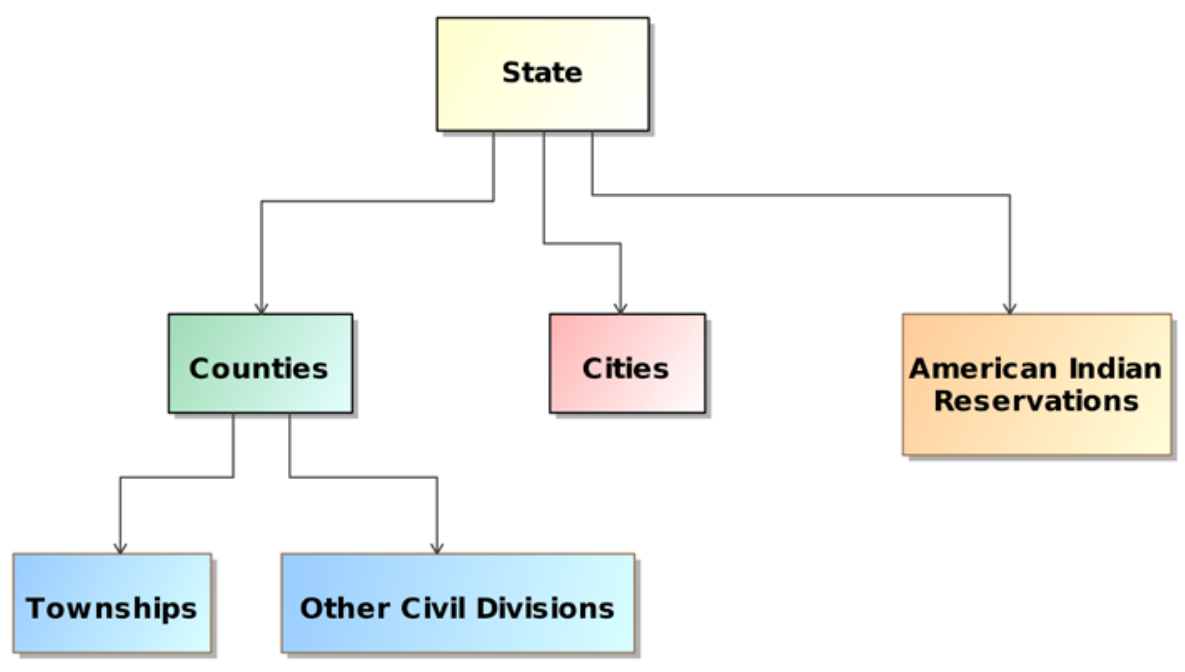

Figure 1 - Governmental-based Geographies

Governmental-based geographies are associated with offices that are elected jurisdiction-wide (such as for Governor, County Clerk, Supervisor, Treasurer, Assessor, Highway Commissioner, etc.) and thus do not require different ballot style areas within the geography for those offices, i.e., all voters in the jurisdiction vote for the office.

Governmental-based geographies do not cross the lines of the precincts that compose them; however cities can change their boundaries through annexations and, in some states, city boundaries can also cross county boundaries. Thus, changes to city boundaries may result in crossing the boundaries of one or more precincts, creating split precincts and requiring a distinct ballot style per split precinct. 


\subsubsection{Political-based Geography}

Political-based geographies are those that tend to be population-based and therefore may change with each U.S. Census every 10 years in a process known as re-districting. Political-based geographies are generally known as electoral districts, where people are elected to an office that has jurisdiction within a specific geography, e.g., a U.S. Congressional district.

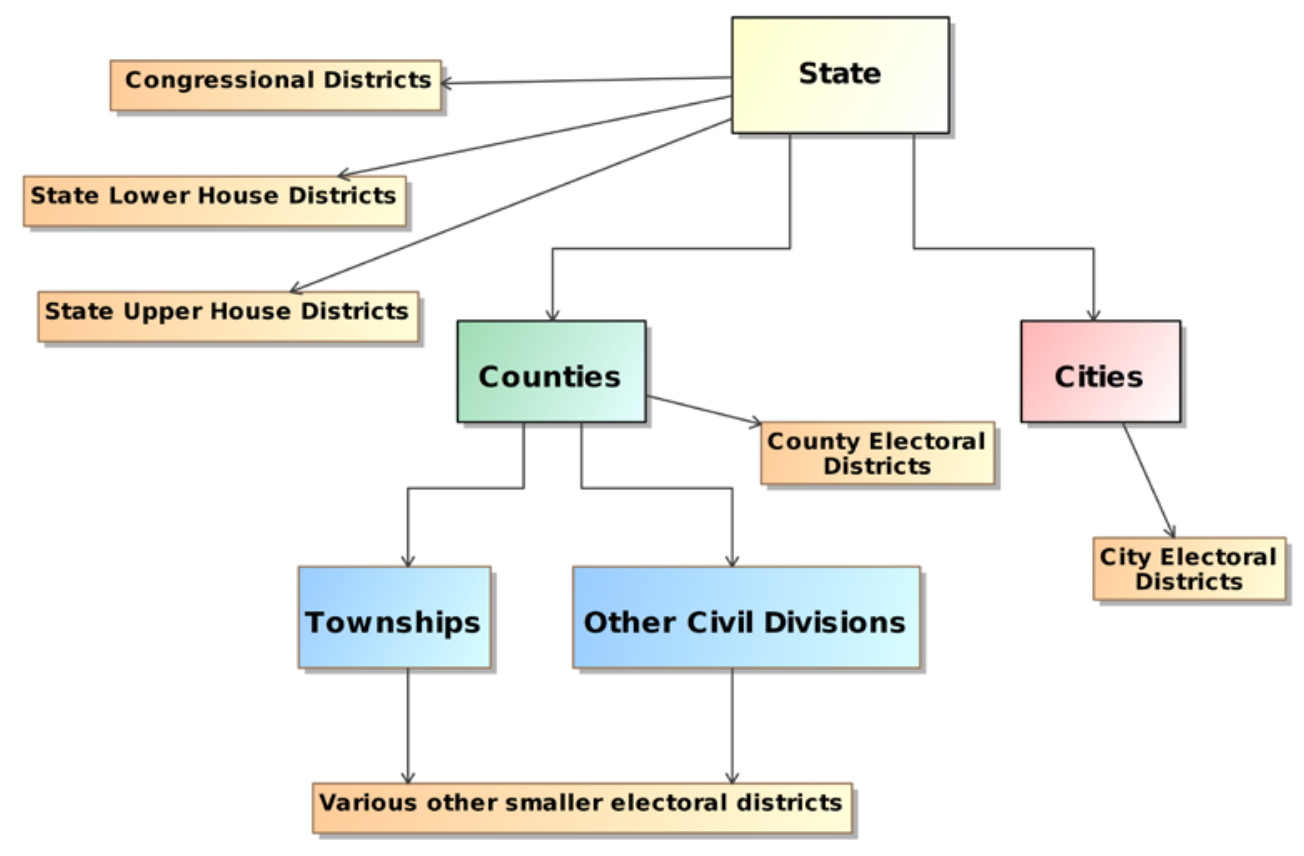

Figure 2 - Political-based Geographies

Figure 2 shows the most common political-based geographies as they interrelate with the governmental-based geographies. Political-based geographies can be categorized as follows:

- U.S. Congressional districts;

- State senate or upper-house districts;

- State house or lower-house districts (in some states, several state house districts combine to form a state senate district);

- County electoral districts;

- City electoral districts (sometimes called Wards); and

- Numerous other forms of electoral districts.

Because electoral districts can change as they are re-drawn, political-based geographies will often divide precincts, creating split precincts and requiring a distinct ballot style per split precinct.

\subsubsection{Administrative-based Geography}

Administrative-based geographies are called thus because their boundaries are determined via 
election or civil administration. Administrative-based geographies include precincts and their various types such as wards, combined precincts, and split precincts. They can be very small, sometimes only applying to several streets or houses or even only a single house along a street. They can involve territory that is non-contiguous in itself, e.g., for some of the taxing and special districts. They can change a number of times throughout a given year, even daily in some cases. Figure 3 shows the basic administrative-based geographies, which can be categorized as follows:

- Election administrative areas;

o Precincts, split precincts, combined precincts, wards;

o Polling places, vote centers;

o Various other ballot style areas;

- Taxing districts, e.g., fire, water, sewer, transit, school, police, hospital, utilities; and

- Special districts, i.e., unique areas brought together for a referendum.

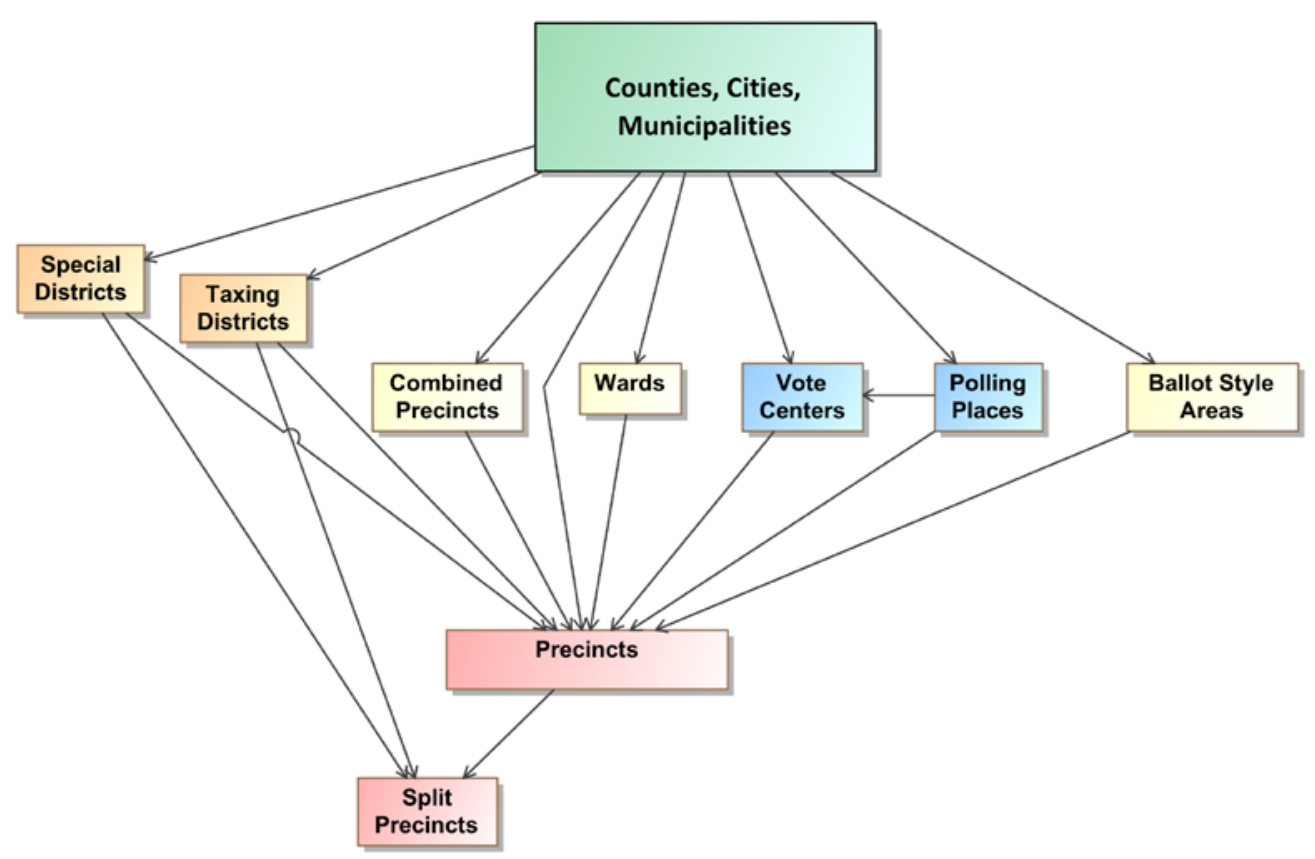

Figure 3 - Administrative-based Geographies

\subsection{Linking the Geopolitical Geographies Together}

As an example of administrative-based geographies and their relationship to political-based and governmental-based geographies, Figure 4 shows the wards and precincts that make up the city of Cambridge, MA, and Figure 5 shows how the wards and precincts in the city compose the U.S. Congressional electoral districts [7]. The wards are implemented as collections of precincts. In general, it is preferred that electoral districts are composed of whole precincts. 


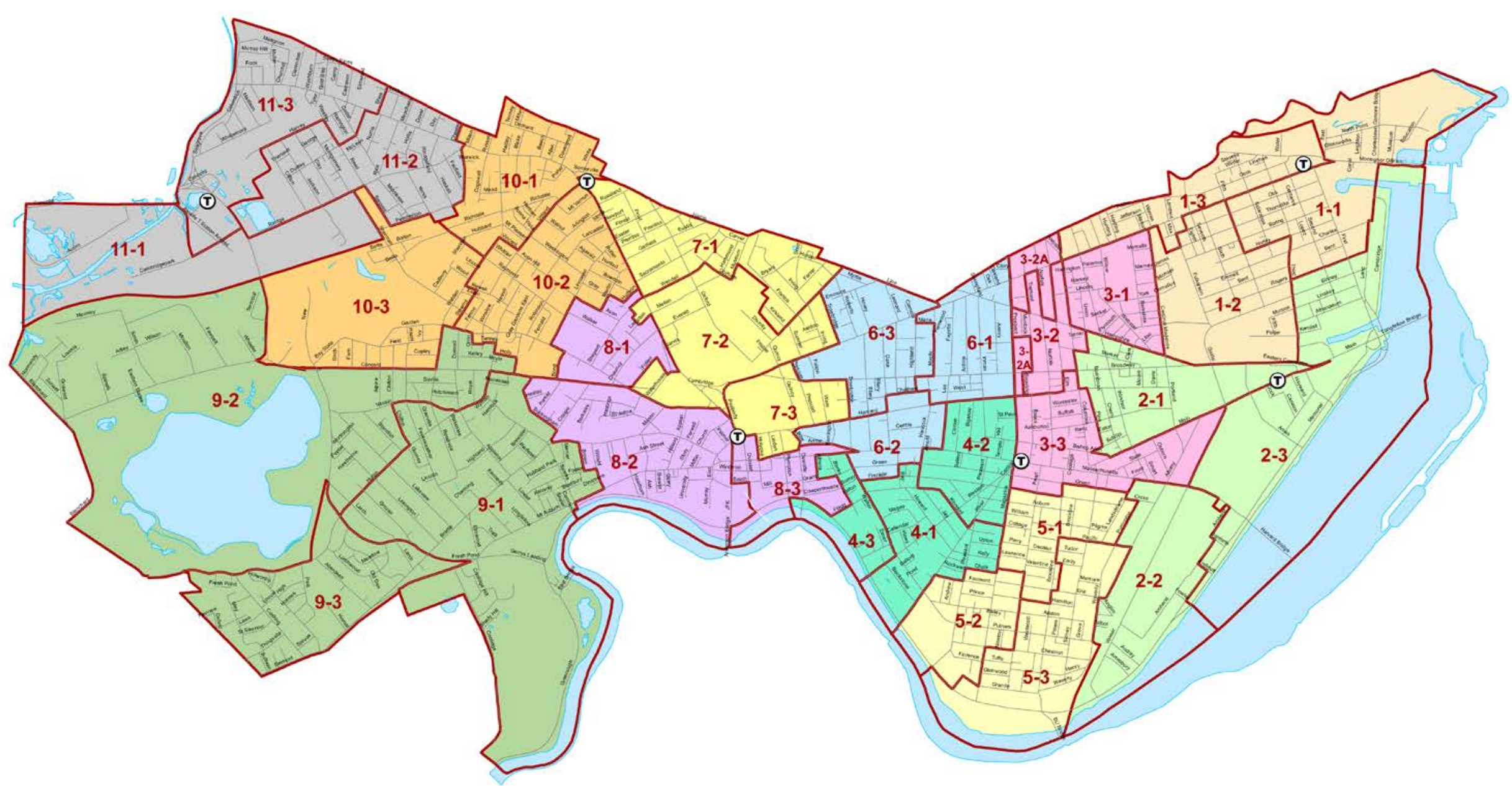

Figure 4 - Ward and Precincts in Cambridge, MA. 


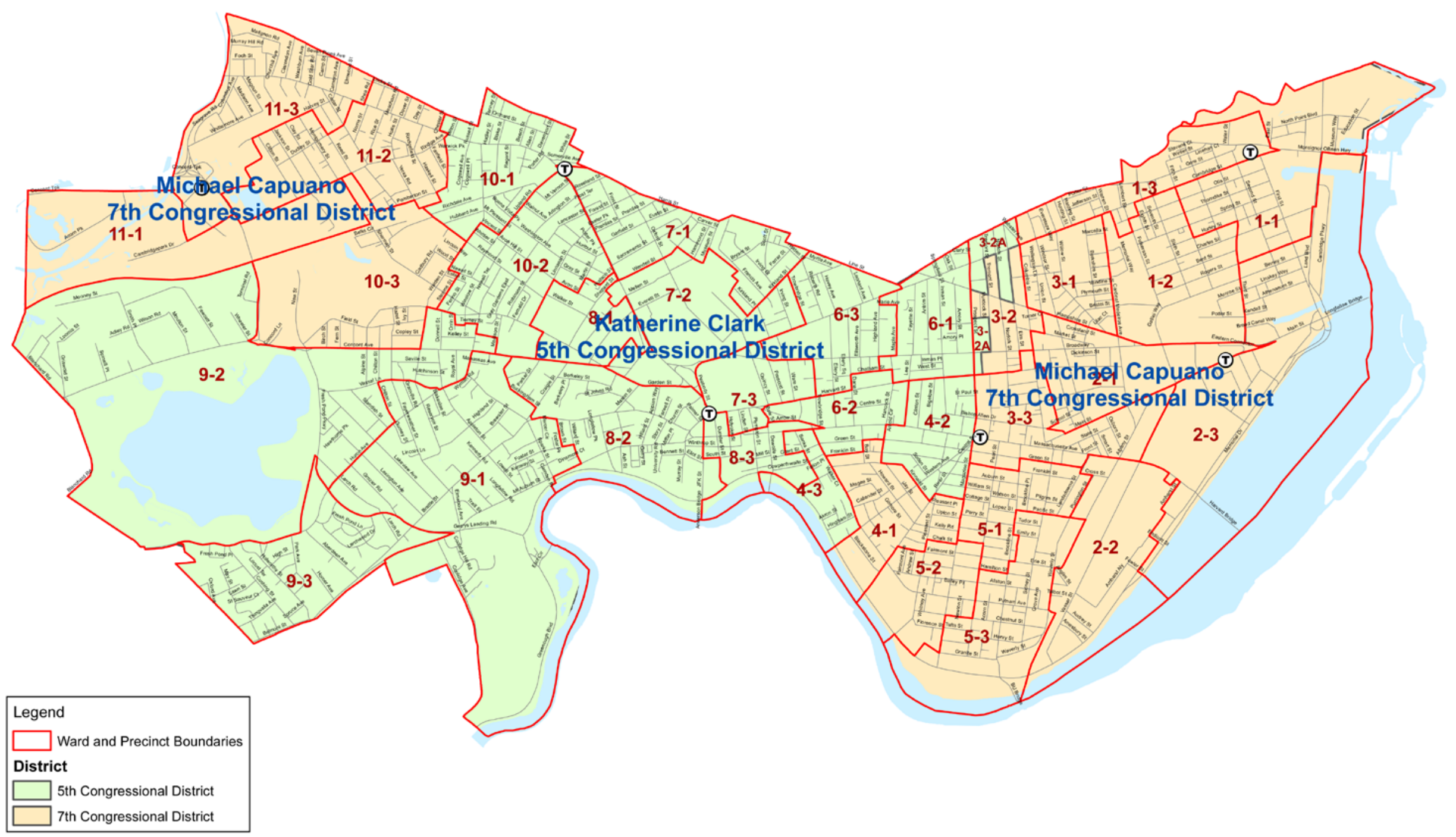

Figure 5 - Districts Overlaying Wards and Precincts in Cambridge, MA. 
In many states, the boundaries of electoral districts may crisscross the precinct boundaries, creating one or more split precincts, with a distinct ballot style per split precinct. Depending on the number of districts and how often they cross the precinct boundaries, the resultant number of ballot styles created may grow substantially beyond the number of whole precincts. It is possible sometimes that, despite best efforts, very low numbers of voters or even just one voter will require a district ballot style.

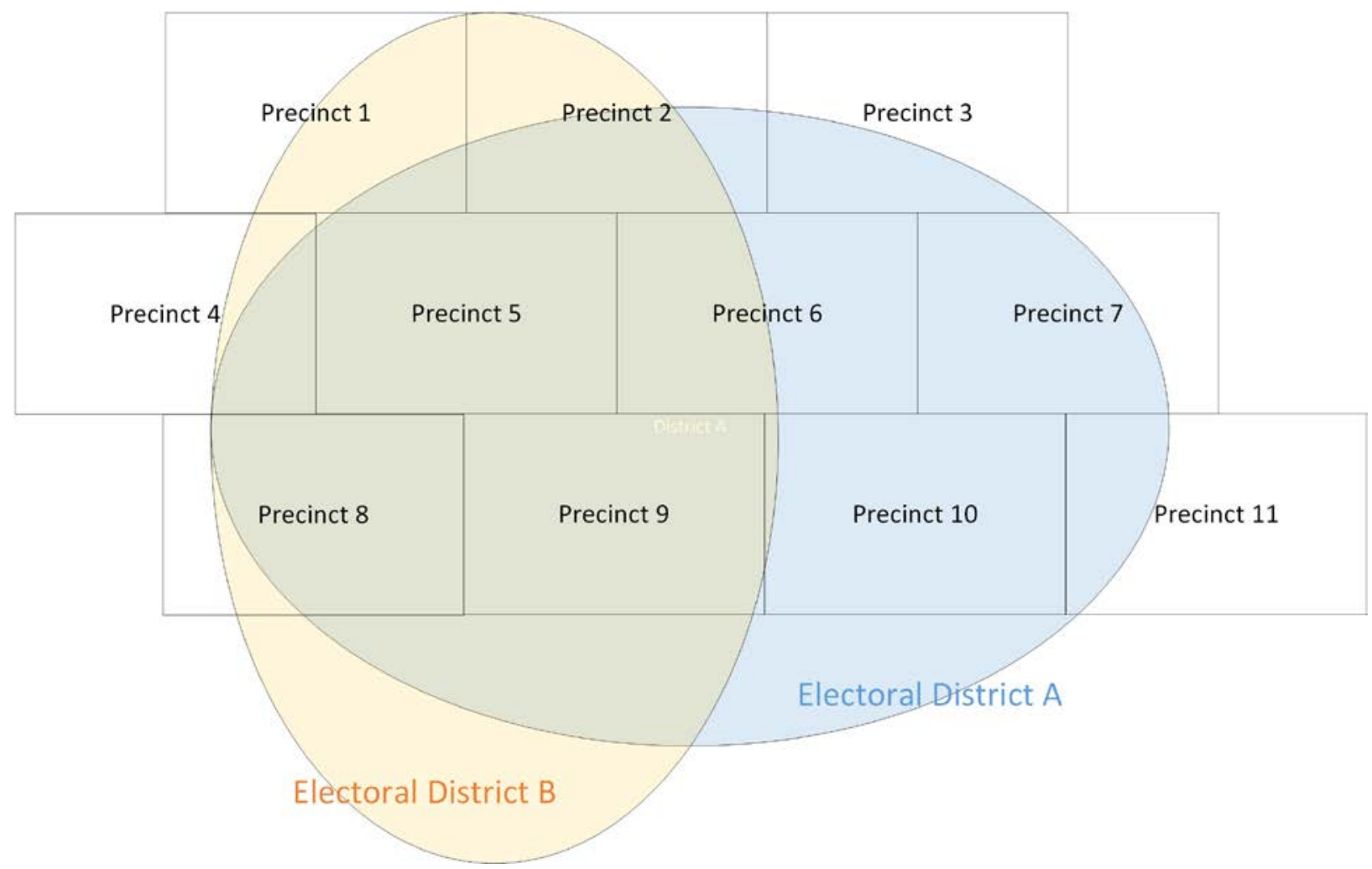

Figure 6 - Overlapping Non-hierarchical Electoral Districts

As an example, Figure 6 shows two electoral districts that overlay a series of precincts. Every time a precinct is not wholly contained within either of the districts, the precinct is split into however many pieces are necessary. Figure 6 shows that a number of the precincts are split in different ways, e.g., Precinct 6 is split into two pieces.

Precinct 6 will thus require two distinct ballot styles, one for each of the splits. To correctly tabulate the votes in the different electoral districts, it will be necessary to know which split of Precinct 6 is contained within each of the two electoral districts.

Precincts can be split as well by changes to the other administrative-based geographies. Adding to the complexity, a number of states now use combined precincts and vote centers on election night, which associate multiple precincts with one polling place. This means that for a vote center handling multiple precincts that themselves may be split, there can be potentially many different ballot styles in use at the vote center, with each voting device needing to display any 
one of the ballots. This adds further complexity and places additional demands on election jurisdictions on their ability to manage and report details of votes on election night and postelection [8].

To make this situation more manageable, some states/counties prefer over time to heal split precincts by combining them with other precincts or generally redrawing the precinct boundaries so that the number of ballot styles is reduced and election management and reporting is less complicated.

\subsection{Geopolitical Geography in the UML Model and XML Schema}

The previous discussion served to show that there are different types of geopolitical geography that overlap each other or behave hierarchically, resulting sometimes in very complex maps and many small geopolitical units that require distinct, different ballot styles. Election officials may spend considerable time in managing this complexity.

Furthermore, each state and sometimes county or city will manage elections differently, using combinations of units such as combined precincts or wards, with specific rules about how the associated contests operate. When one combines (a) the complexities of geopolitical geography with (b) the different election rules employed in the U.S. states and territories, one sees that running an election can be an extremely complicated endeavor. Election results reporting mirrors this complexity.

It is important to note that the different geographies form relationships much like a lattice, in which objects can be related in non-hierarchical ways. The UML model and XML schema implement geopolitical geography in this way using an object that can be linked with other objects depending on the type of geopolitical geography. In the UML model, this object is referred to as the GpUnit (short for 'Geopolitical Unit”) class, and in the XML schema it is called the <GpUnit> element. GpUnits can model a district, or county, or precinct, etc., and can be linked to each other to mirror the real-world geopolitical geography of the reporting jurisdiction.

GpUnits can be linked hierarchically when modeling jurisdictional geographies. To model a jurisdiction that runs/reports on elections, the lowest-level GpUnits, i.e., precincts, will be children of the election-running GpUnit, say a city or county or state.

District GpUnits need to be linked to the precinct and/or split precinct GpUnits that compose them. The precincts and split precincts thus link the jurisdictional and district GpUnits together, as shown below in Figure 7 (and described in greater detail in section 5.2). The wards are the children of the combined precincts, and so forth on up to the state. The precincts and split precincts are also the children of the districts that they compose. 


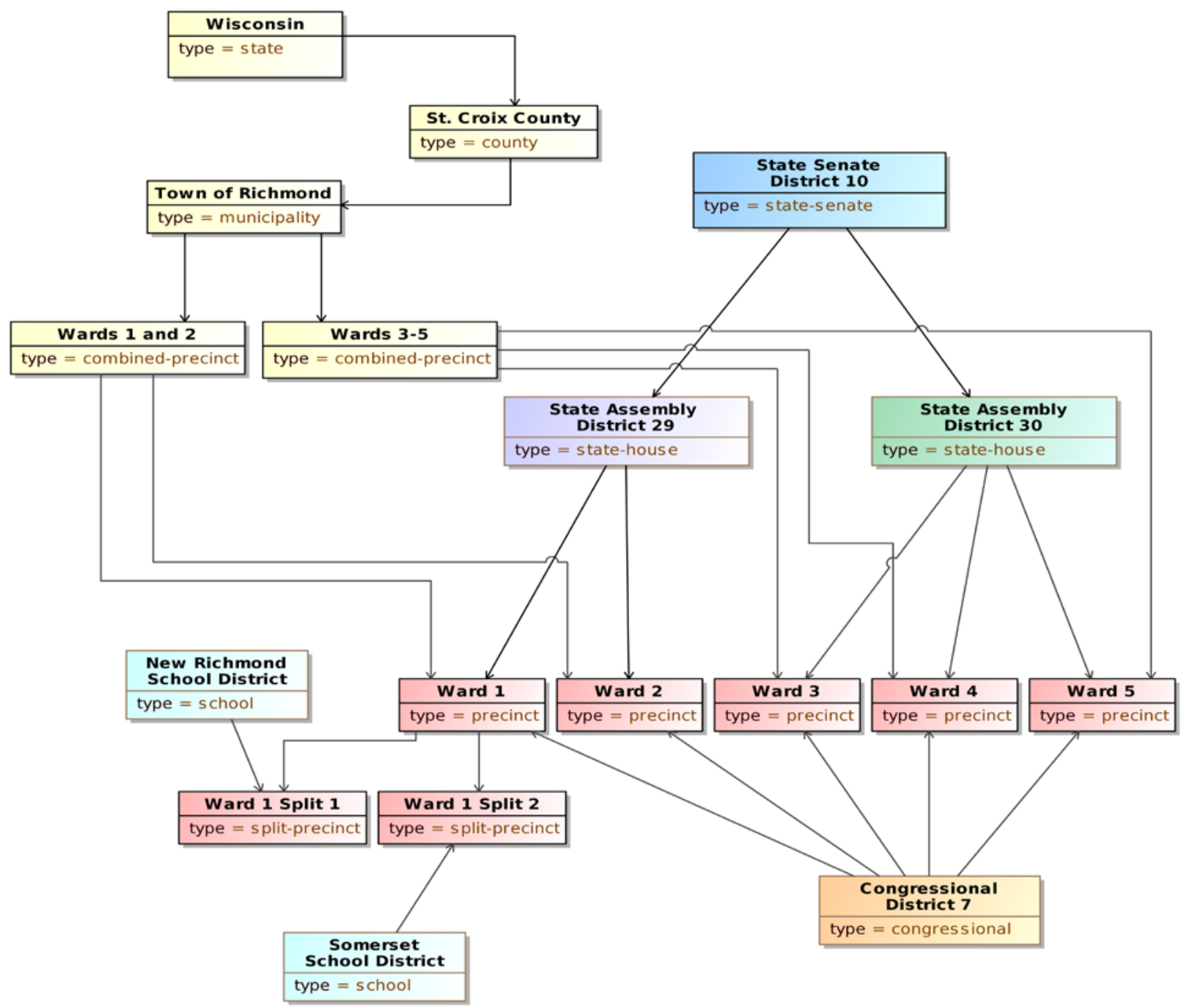

Figure 7 - GpUnit Structural Hierarchies 


\section{Use Cases and UML Model}

This section describes the three use cases for pre-election setup and post-election results reporting included in this specification, and then describes the UML model that implements the use cases and was used to generate the XML schema. This section is provided for background and to assist in understanding how the model and schema are structured and used.

\subsection{Use Cases}

This section describes the three use cases for the following phases of pre-election setup and postelection results reporting:

1. Pre-election - for reporting of election data about offices, political parties, and the geopolitical geographies associated with the reporting jurisdiction, as well as any election-specific information such as the contests, candidates, or ballot measures;

2. Election night - for reporting of election results to the public during the election or on election night and for internal state rolls-ups of election results between and among election management systems; and

3. Post-election - for reporting the final results compiled during the post-election canvass, that is, when all results are re-examined and re-tabulated to ensure correctness.

\subsubsection{Pre-Election Use Case}

The pre-election use case enables election officials to report on a variety of election-related data; the data could include information about a specific upcoming election or it could provide more general information about the reporting jurisdiction so as to determine whether the election data is accurate and organized correctly or to convey information to the general public about contests and ballot information in an upcoming election. Pre-election information may come from any of the databases or devices that an election jurisdiction uses to manage elections, including voter registration databases, ballot programming systems, candidate filing systems, EMSs, campaign finance systems, etc.

The data for the pre-election use case includes the following:

- Pre-election reports in a variety of formats, including:

o As one complete file or a sequence of files;

o As additions to previous reports; and

o As corrections to previous reports;

- Jurisdictions, districts, offices, precincts and voting locations within the scope of the reporting jurisdiction and how they are organized;

- Political party information;

- Offices associated with contests and districts; 
- Information about persons relevant to the jurisdiction such as authorities, candidates, office holders, etc.;

- Election type, date, place, jurisdiction, authority information, registered voters. The type of election could include:

o Open and closed partisan primaries;

o Runoffs;

o Special elections; and

o General elections;

- Ballot measures and contests, including retention contests;

- Candidates on the ballot and their associated party affiliations and contact information;

- Offices associated with the contests and electoral districts;

- Vote-capture device types or the specific devices associated with polling places; and

- Ballot styles containing contest and candidate information in the order as they appear on the ballot at specific precincts or split precincts.

\subsubsection{Election Night Reporting Use Case}

The election night reporting use case addresses aggregated, contest-level reporting of election results. Depending on a jurisdiction's capabilities, it could include further details such as precinct-level vote and ballot counts and breakdowns of counts by device type and ballot type.

The election night reporting use case involves election reporting managed in two primary ways:

1. By the counties (or cities, etc.) reporting directly to the public/media and, possibly, also reporting upward to the state, or

2. By the counties reporting upward to the state and the state reporting directly to the public/media.

When reporting upward to the state, election results are reported from local jurisdictions to the city or county or state level, where the results continue to be aggregated in a process referred to as rolling up the votes and creating state or county roll-ups. As part of this process, internal, intermediate reports from different EMSs or databases need to be combined. The roll-ups are then formatted and released to the media and public during election night until all results from the local jurisdictions are accounted for. The results are, at this stage, considered as unofficial.

The data for election night reporting includes the following:

- Any information from the pre-election use case;

- Aggregated contest results for offices and ballot measures, including:

o Votes reported in the contest;

o Votes reported for each candidate(s) or selection(s) in the contest;

o Overvotes reported for the contest; and

o Undervotes reported for the contest;

- Further vote details as possible to report, including: 
o Breakdowns of votes by various reporting units such as precinct or split precinct;

o Vote counts and ballot counts broken down by ballot type (absentee, early, etc.) and type of device (electronic, optical scan, etc.); and

o Additional counts as available, e.g., the number of votes cast by party or the number of straight party selections.

\subsubsection{Post-Election Reporting Use Case}

The post-election reporting use case addresses the updates to the election night unofficial results. Post-election results contain greater detail than election night results, including results by reporting unit, by counts of different types of ballots (early, absentee, provisional, etc.), and by the type of vote-capture device used by voters to cast their ballots. They may also include results that were not available for election night reporting, such as absentee ballots received too close to the election day, or provisional ballots. This use case is of interest especially by analysts and media performing detailed analysis of election results and other items of interest such as the methods people are choosing to vote (e.g., favoring absentee over in-person) or provisional voting rejection rates.

The data for post-election night reporting includes:

- Any information from the pre-election and election night reporting use cases;

- Ballot measure and contest vote counts, including:

o By geopolitical units of geography (e.g., county, township, city, precinct, etc.);

o By type of ballot/voting (e.g., absentee, early, in-person, provisional);

0 By type of voting equipment used (e.g., electronic, optical scan, etc.); and

o By overvotes and undervotes for each contest;

- Ballot measure and contest summaries broken down as per the vote counts, but also including the number of ballots on which the ballot measure or contest appeared; and

- Precinct summaries broken down as per the vote counts.

\subsection{UML Model}

This section presents the UML model that was structured to implement the use cases. It includes a class diagram that shows a picture of the model and an overview of how to read the relationships between the classes so as to understand how the model and XML schema are structured.

The UML model represents a format-independent description of the data required by the three use cases of the specification. Its primary benefit is that it unambiguously defines and describes the data elements and how they are related without requiring readers to know the technical details of any particular data format implementation such as XML. By using a model-based approach, the resultant data format is more likely to be well-structured and more tolerant to modifications. The data format can be generated from the model using commercial tools, thus if 
changes need to be made to a format, the model can be changed and the format can be regenerated.

Figure 8 shows a high-level view of the class diagram, minus its attributes and certain ancillary classes (the complete diagram is available for download, see Appendix C-References).

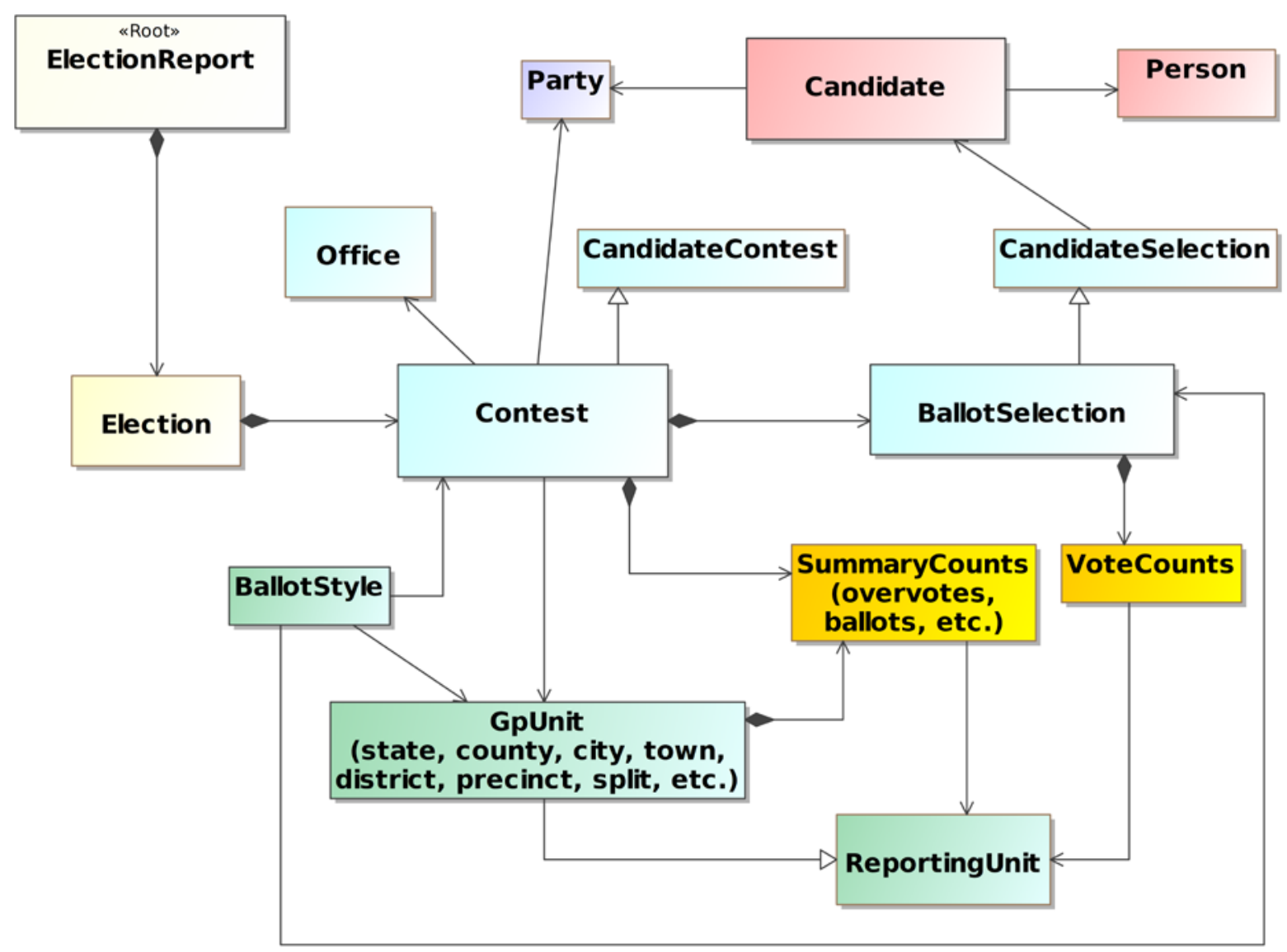

Figure 8 - Simplified UML Class Diagram

\subsubsection{Major Classes}

Each class represents a major data element, e.g., Contest, Candidate, or Party, and classes that are highly related to each other are shown in the same color. When XML is generated from the UML model, the classes in the model get generated as major XML elements, e.g., the UML Candidate class results in the XML <Candidate $>$ element. The major classes shown in Figure 8 are:

- ElectionReport - the root class, includes attributes describing the type of report, when generated, etc.; 
- Election - for a specific election, includes attributes describing the type of election, date, etc.;

- GpUnit - short for “Geopolitical Unit,” for describing units of geopolitical geography so that they can be associated with contests, offices, ballot styles, and election results. There are two major types of GpUnits:

1. ReportingUnit - for jurisdictions, districts, precincts, and other units for which election results can be associated; and

2. ReportingDevice - for types of voting devices or specific voting devices for which election results can be associated (not shown in Figure 8);

- Office - for political offices that are associated with contests and electoral districts and current office holders;

- Party - for political parties associated with the reporting jurisdiction and for associating the parties with candidates and contests;

- Person - for address and contact information for persons associated with the reporting jurisdiction, including boards of authorities, candidates, current office holders, or other election-related officials;

- Contest - for contests and for linking together the major elements needed for contests such as candidates and ballot selections and election results. There are four types of Contests:

1. CandidateContest - for contests involving candidates;

2. BallotMeasureContest - for contests involving ballot measures (not shown in Figure 8);

3. PartyContest - for straight-party selection (not shown in Figure 8); and

4. RetentionContest - for judicial or other types of retention contests (not shown in Figure 8);

- Candidate - for describing candidates so that they can be associated with contests;

- BallotSelection - for describing the types of ballot selections in a contest and associating them with election results:

1. BallotMeasureSelection - used if the ballot selection is for a ballot measure (not shown in Figure 8);

2. CandidateSelection - used if the ballot selection is for a candidate; and

3. PartySelection - used if the ballot selection is for a party as in straight party selections (not shown in Figure 8);

- VoteCounts - used for the vote counts associated with a ballot selection;

- SummaryCounts - for describing summary counts of overvotes and undervotes, writeins, and ballot counts associated with (a) a contest or (b) geopolitical units such as precincts; and

- BallotStyle - for describing the contests and ballot selections on a ballot and linking them to geopolitical units such as precincts.

The attributes associated with the UML classes correspond closely to the XML schema's attributes and sub-elements associated with the major elements, described in Section 4. 


\subsubsection{Relationships between Classes}

The major classes in the UML model result in major elements in the XML schema, and the different types of relationships between the UML classes determine how the XML elements are structured (linked) in the schema. There are three types of relationships between the classes:

Directed Composition: see Figure 9, ElectionReport and Election, read as, "An election report is composed of elections." In the XML schema, the $<$ Election $>$ element will be generated as a sub-element of the <ElectionReport> element. A directed composition relationship has a closed diamond at one end and an arrow pointing to the composing class:

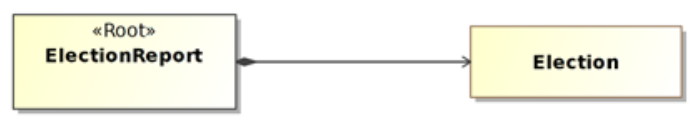

Figure 9 - Directed Composition Example

Is a Type of or Instance of: see Figure 10, Contest and CandidateContest, read as, "A candidate contest is a type of contest." Contest is an abstract class; it is "implemented" by its concrete classes such as CandidateContest. In the XML schema, Contest will be generated as an abstract XML element and serve as an extension base to the <CandidateContest $>$ element. An instance relationship has an open triangle at one end, pointing from the concrete class to the abstract class:

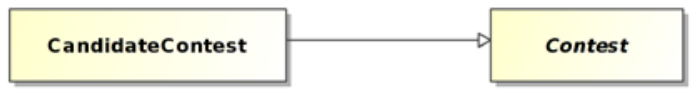

Figure 10 - "Is a Type of" Example

Directed Association: see Figure 11, Candidate and Party, read as, "A candidate is associated with or linked to a party." In the XML schema, the <Candidate $>$ element will include a $<$ PartyId $>$ element, which will contain an identifier associated with a <Party $>$ element. A directed association has an arrow at one end, goes in one direction, and serves to link the class to another associated class, e.g., the party linked to the candidate:

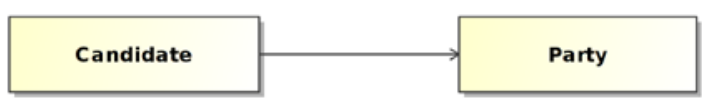

Figure 11 - Directed Association Example

The generation of XML elements from the UML model is discussed more specifically in section 5.1.2.

For the directed composition associations in Figure 8, one can see that ElectionReport is composed of Election, GpUnit, Office, Party, and Person. Election is composed of BallotStyle, Candidate, and Contest. Contest is composed of BallotSelection and SummaryCounts. 
BallotSelection is composed of VoteCounts. GpUnit is also composed of SummaryCounts.

For the "is a type of" relationships in Figure 8, one can see that CandidateContest is a type of Contest. ReportingUnit is a type of GpUnit. CandidateSelection is a type of BallotSelection.

Lastly, for the directed associations in Figure 8, one can see that many of the classes are associated with each other, as one would expect. For example, Party is associated with (or linked to) Candidate just as a political party would be associated with or linked to a candidate. Candidate is associated or linked to CandidateSelection, just as a selection on the ballot in a candidate contest would be for a specific candidate. The XML generation is more complicated for directed associations and is discussed in greater detail in section 5.1.3. 


\section{$4 \quad$ XML Schema}

This section contains documentation and discussion of the features included in the XML schema.

In the sections below, an element or an enumeration name is denoted using italics and angle brackets, e.g., <ElectionReport $>$ or $<$ ReportingUnitType $>$. Attributes and enumeration values are in italics, e.g., label or geo-json. An element is sometimes referred to as a "sub-element" when it is included in another element, e.g., <Election $>$ is a sub-element of <ElectionReport>. "Includes" is used to denote that an element contains another element as a sub-element, e.g., <ElectionReport $>$ includes <Election $>$. "References" is used to denote one element linking to a second element via the second element's objectId attribute, e.g., $<$ Candidate $>$ references $<$ Party $>$ using the $<$ PartyId $>$ sub-element.

\subsection{Schema Stylistic Conventions}

The XML schema was written observing the following stylistic conventions:

- Element, attribute, enumeration, and primitive names observe variations of "CamelCase" conventions ${ }^{2}$, that is:

0 Element and enumeration and primitive names begin with a capital letter and names that consist of multiple words are concatenated and each word begins with a capital letter, thus "CamelCase". For example, <ElectionReport>;

0 Attribute names begin with a non-capital (lower-case) letter and names that consist of multiple words are concatenated, with the first word beginning in a non-capital letter and subsequent words beginning in a capital letter, thus "camelCase". For example, objectId; and

o Enumeration value names are in non-capital letters, and names that consist of multiple words are separated by hyphens. For example, 1-of-n;

- Element, attribute, and enumeration value ordering is generally alphabetical, with the following exceptions:

$0<$ EndDate $>$ follows element names such as $<$ StartDate $>$, or $<$ OtherType $>$ follows $<$ Type>;

o The objectId attribute comes first, followed by the other attributes in alphabetical order; and

o If there is an enumeration value of other, it comes last in the list of values.

\subsection{Elements and Complex Types}

The following sections deal with major elements and complex types in the schema.

\footnotetext{
${ }^{2}$ See https://en.wikipedia.org/wiki/CamelCase.
} 


\subsubsection{The <AnnotatedString> Complex Type}

Used as a type for character strings; it adds a 16-character annotation to a character string, for example:

$<$ Email annotation="Work" $>$ john.a.smith@26003.org $</$ Email $>$

$<$ Phone annotation ="Cell" $>304-243-1234</$ Phone $>$

Table 4.1 - Attributes for <AnnotatedString>

\begin{tabular}{|c|c|c|l|}
\hline Attribute & Required & Type & \multicolumn{1}{|c|}{ Attribute Description } \\
\hline annotation & no & ShortString & $\begin{array}{l}\text { An annotation of up to 16 characters associated with a } \\
\text { character string. }\end{array}$ \\
\hline
\end{tabular}

Schema Definition:

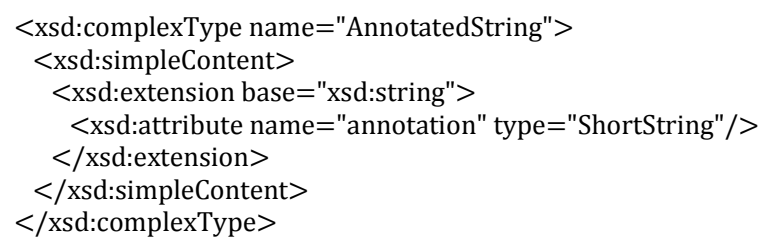




\subsubsection{The <BallotSelection> Element and Extension Base}

Used for the ballot selections in a contest (e.g., for candidates, for ballot measures) and to generally link them to vote counts. <Contest $>$ includes <BallotSelection $>$.

$<$ BallotSelection $>$ is an abstract element with three xsi:types that get used according to the type of contest:

- $\quad<$ BallotSelection xsi:type="BallotMeasureSelection" $>$, used if the contest type is for a ballot measure, including for retentions (see section 4.2.2.1);

- $\quad<$ BallotSelection xsi:type="CandidateSelection" $>$, used if the contest type is for one or more candidates, to link the ballot selection to the candidate elements and endorsement parties (see section 4.2.2.2); and

- $\quad<$ BallotSelection xsi:type="PartySelection" $>$, used if the contest type is for a party, e.g., for a straight party contest (see section 4.2.2.3).

$<$ BallotSelection $>$ includes $<$ VoteCounts $>$ for associating vote counts with the ballot selection. $<$ BallotSelection > "wraps" occurrences of < VoteCounts > elements in a container element for the purpose of making large instance files easier to manipulate in XML viewers and editors.

$<$ SequenceOrder $>$ is included to specify an ordering for the ballot selections for purposes of display only. The original ballot ordering can be preserved, however, by using the $<$ BallotStyle $>$ element (see section 4.2.3).

Table 4.2 - Attributes for <BallotSelection>

\begin{tabular}{|c|c|c|l|}
\hline Attribute & Required & Type & \multicolumn{1}{|c|}{ Attribute Description } \\
\hline objectId & yes & xsd:ID & $\begin{array}{l}\text { Unique internal identifier used by other element(s) to } \\
\text { reference this element. }\end{array}$ \\
\hline
\end{tabular}

Table 4.3 - Elements for <BallotSelection>

\begin{tabular}{|c|l|l|l|}
\hline Element & Multiplicity & Type & \multicolumn{1}{|c|}{ Element Description } \\
\hline$<$ SequenceOrder $>$ & 0 or 1 & xsd:integer & $\begin{array}{l}\text { Order in which the candidate is listed on the } \\
\text { ballot for purposes of results display. If not } \\
\text { present, no order is assumed. }\end{array}$ \\
\hline$<$ VoteCountsCollection $>$ & 0 or more & & $\begin{array}{l}\text { Wrapper for collections of < } \\
\text { elementeCounts }>\end{array}$ \\
\hline$<$ VoteCounts $>$ & 1 or more & VoteCounts & $\begin{array}{l}\text { For associating votes with a ballot selection in } \\
\text { a contest. }\end{array}$ \\
\hline
\end{tabular}

Schema definition: 


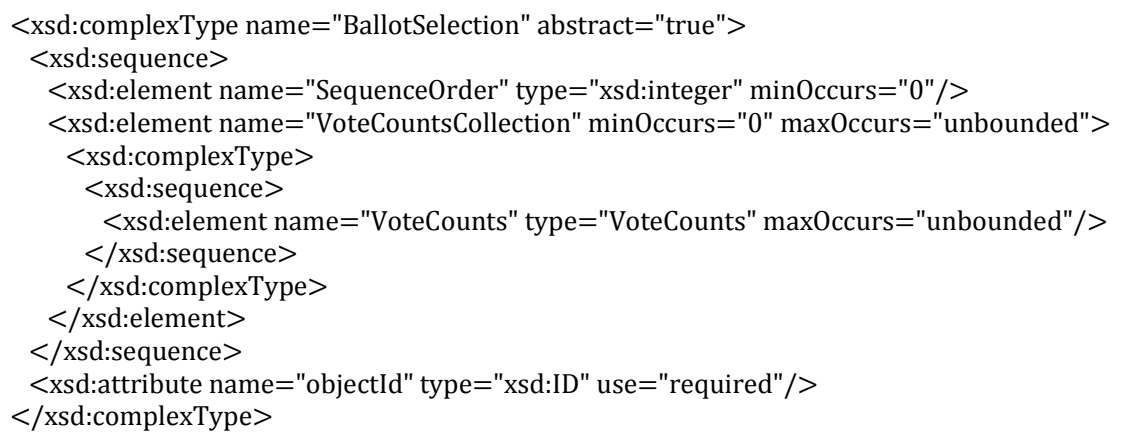




\subsubsection{The xsi:type=BallotMeasureSelection}

For a ballot selection in a ballot measure contest. Because judicial or other retention contests are often treated like ballot measure contests, this element can be used also for retention contests. It inherits the attributes and elements of <BallotSelection > (see Table 4.2 and Table 4.3). Its syntax is:

$<$ BallotSelection xsi:type="BallotMeasureSelection"... />

Table 4.4 - Elements for <BallotSelection xsi:type="BallotMeasureSelection">

\begin{tabular}{|c|c|c|c|}
\hline Element & Multiplicity & Type & \multicolumn{1}{c|}{ Element Description } \\
\hline <Selection> & 1 & InternationalizedText & $\begin{array}{l}\text { Contains the text used to indicate a vote for or } \\
\text { against the ballot measure, e.g., "yes", "no”. }\end{array}$ \\
\hline
\end{tabular}

Schema definition:

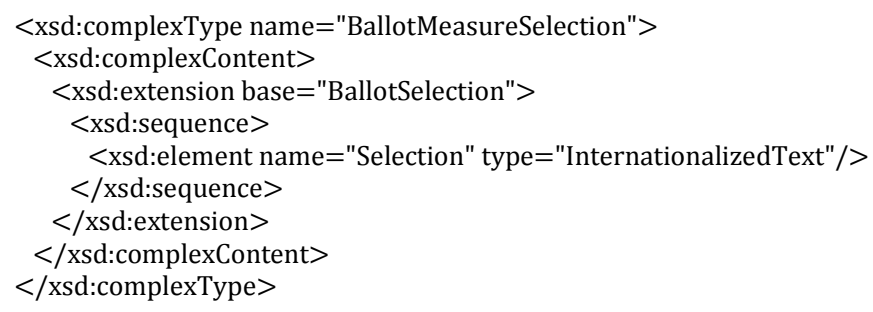




\subsubsection{The $x$ si:type=CandidateSelection}

For the ballot selections in a candidate contest, including for write-ins. It inherits the attributes and elements of <BallotSelection $>$ (see Table 4.2 and Table 4.3). References to multiple $<$ Candidate > elements can be included if necessary, e.g., when the ballot selection would be for a ticket of candidates (unless the ticket itself is defined as a candidate). Its syntax is:

$<$ BallotSelection xsi:type="CandidateSelection"... $/>$

$<$ EndorsementPartyIds $>$ is used to reference any associated endorsement parties other than the specific party of the candidate (<Candidate $>$ references $<$ Party $>$ for that purpose). For example, if a candidate of one party is also endorsed by a second party, use $<$ EndorsementPartyIds $>$ to reference the second party. A second example would be for ballot fusion as used in some states, where the same candidate is listed multiple times in the same contest, but with different endorsement parties.

When multiple candidates are referenced for a ticket and the ordering of the candidates is important to preserve, it is expected that the generating application will list the references to $<$ Candidate $>$ elements within <CandidateIds $>$ according to the ordering scheme in place. For example, if the contest is for a ticket in which each candidate is associated with a different office, then the order of the candidates should match the same ordering of the <office $>$ element references within $<$ OfficeIds $>$ in the <Contest xsi:type $=$ "CandidateContest"...$>$ element (see section 4.2.6.2).

Table 4.5 - Elements for <BallotSelection xsi:type="CandidateSelection">

\begin{tabular}{|c|c|c|l|}
\hline Element & Multiplicity & Type & \multicolumn{1}{|c|}{ Element Description } \\
\hline <CandidateIds> & 1 & xsd:IDREFS & $\begin{array}{l}\text { Unique identifiers for one or more <Candidate }> \\
\text { elements. For associating a candidate with the } \\
\text { candidate selection on the ballot and for cases } \\
\text { where the ballot selection is for multiple } \\
\text { candidates, e.g., a ticket. }\end{array}$ \\
\hline <EndorsementPartyIds> & 0 or 1 & xsd:IDREFS & $\begin{array}{l}\text { Unique identifiers for one or more }<\text { Party }> \\
\text { element. For associating one or more endorsing } \\
\text { parties with the candidate selection. }\end{array}$ \\
\hline IsWriteIn & 0 or 1 & xsd:boolean & $\begin{array}{l}\text { Indicates whether the candidate is a write-in, } \\
\text { e.g., true or false. Assumed to be false if not } \\
\text { present. }\end{array}$ \\
\hline
\end{tabular}




\section{Schema definition:}

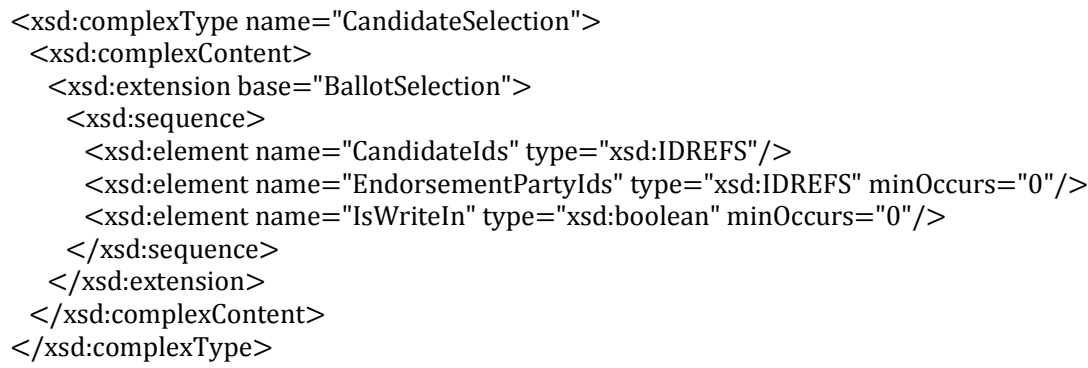




\subsubsection{The xsi:type=PartySelection}

For a ballot selection involving a party such as for a straight party selection on the ballot. It inherits the attributes and elements of <BallotSelection> (see Table 4.2 and Table 4.3). Its syntax is:

$<$ BallotSelection xsi:type="PartySelection" $\ldots />$

Table 4.6 - Elements for <BallotSelection xsi:type="PartySelection">

\begin{tabular}{|c|c|c|l|}
\hline Element & Multiplicity & Type & \multicolumn{1}{|c|}{ Element Description } \\
\hline$<$ PartyIds $>$ & 1 & xsd:IDREFS & $\begin{array}{l}\text { Unique identifier for one or more }<\text { Party }> \\
\text { elements. For associating one or more parties } \\
\text { with the party selection. }\end{array}$ \\
\hline
\end{tabular}

Schema definition:

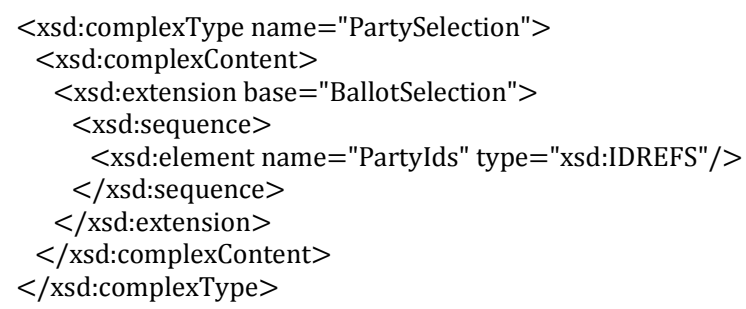




\subsubsection{The <BallotStyle> and <OrderedContest> Elements}

For defining a ballot style composed of contests and their ballot selections, and associating the ballot style with a political party, a reference to an image of the ballot, and a reference to the a precinct or other geopolitical unit that the ballot is unique to. $<$ Election $>$ includes $<$ BallotStyle $>$.

$<$ BallotStyle $>$ references < OrderedContest $>$ to include contests on that ballot style and the ballot selections associated with each contest (see section 4.2.3.1). To preserve any rotation associated with the ballot, it is expected that the generating application will list the occurrences of $<$ OrderedContest $>$ in the order as on the ballot for the associated geopolitical unit.

$<$ BallotStyle $>$ includes < GpUnitIds $>$ to reference one or more <GpUnit > elements defined for the associated precincts or split precincts. If the ballot style is associated with multiple precincts (or other geographies), multiple references to the precinct <GpUnit> elements can be included, e.g.,

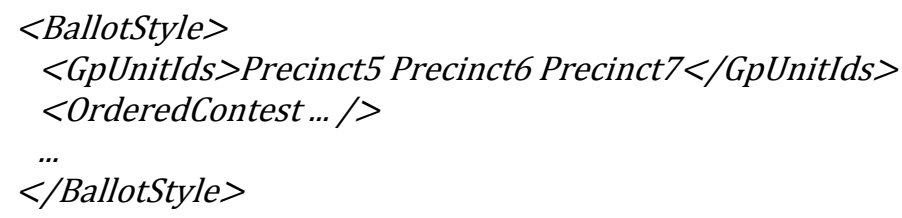

When including < ExternalIdentifiers $>$, if the type is not listed in enumeration <IdentifierType>, use other and include the type (that is not listed in the enumeration) in OtherType.

Table 4.7 - Attributes for <BallotStyle>

\begin{tabular}{|c|c|c|l|}
\hline Attribute & Required & Type & \multicolumn{1}{|c|}{ Attribute Description } \\
\hline objectld & yes & xsd:ID & $\begin{array}{l}\text { Unique internal identifier used by other element(s) to reference } \\
\text { this element. }\end{array}$ \\
\hline
\end{tabular}

Table 4.8 - Elements for <BallotStyle>

\begin{tabular}{|c|c|c|l|}
\hline Element & Multiplicity & Type & \multicolumn{1}{|c|}{ Element Description } \\
\hline$<$ ExternalIdentifiers $>$ & 0 or 1 & ExternalIdentifiers & $\begin{array}{l}\text { For associating an ID with the ballot } \\
\text { style. }\end{array}$ \\
\hline$<$ GpUnitIds $>$ & 1 & xsd:IDREFS & $\begin{array}{l}\text { Unique identifier for one or more } \\
<\text { GpUnit }>\text { elements. For associating } \\
\text { specific geopolitical units with the } \\
\text { ballot style. }\end{array}$ \\
\hline$<$ ImageUri> & 0 or more & xsd:anyURI & URI for a ballot image. \\
\hline$<$ OrderedContest $>$ & 0 or more & OrderedContest & $\begin{array}{l}\text { For associating contests in the order as } \\
\text { listed on ballot. }\end{array}$ \\
\hline$<$ PartyIds $>$ & 0 or 1 & xsd:IDREFS & $\begin{array}{l}\text { Unique identifier for one or more } \\
<\text { Party }>\text { elements. For associating one }\end{array}$ \\
\hline
\end{tabular}




\begin{tabular}{|c|c|c|c|}
\hline Element & Multiplicity & Type & Element Description \\
\hline & & & or more parties with the ballot style. \\
\hline
\end{tabular}

\section{Schema definition:}

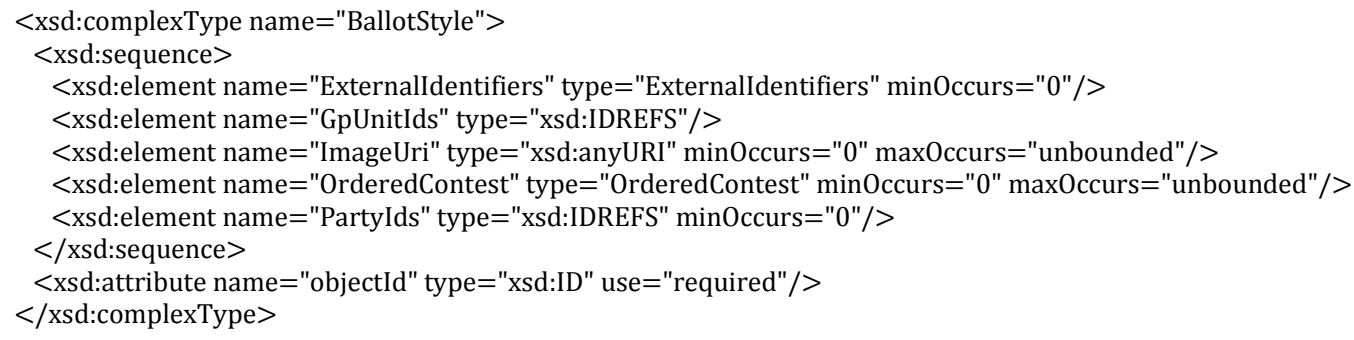




\subsubsection{The <OrderedContest> Element}

Associates a reference to a contest with references to the contest's ballot selections. <BallotStyle> includes < OrderedContest> for each contest on the ballot. To preserve any rotation associated with the ballot, it is expected that the generating application will list the occurrences of references to <BallotSelection $>$ elements within < OrderedBallotSelectionIds $>$ in the order as on the ballot for the associated geopolitical unit.

Table 4.9 - Elements for <OrderedContest>

\begin{tabular}{|c|c|c|l|}
\hline Element & Multiplicity & Type & \multicolumn{1}{|c|}{ Element Description } \\
\hline <ContestId & 1 & xsd:IDREF & $\begin{array}{l}\text { Unique identifier for a <Contest }> \\
\text { element. For associating a } \\
\text { contest on the ballot. }\end{array}$ \\
\hline <OrderedBallotSelectionIds $>$ & 0 or 1 & xsd:IDREFS & $\begin{array}{l}\text { Unique identifier for one or more } \\
<\text { BallotSelection }>\text { elements. For } \\
\text { associating ballot selections with } \\
\text { the contest. }\end{array}$ \\
\hline
\end{tabular}

Schema definition:

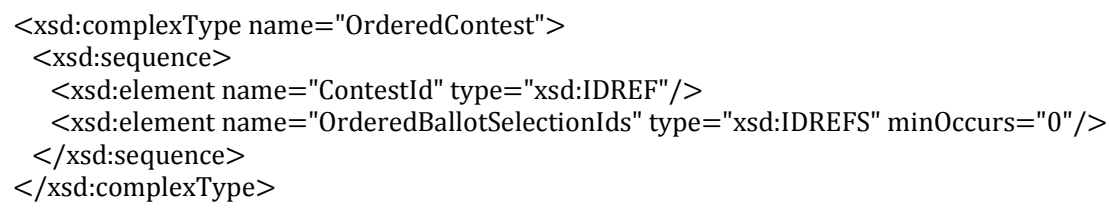




\subsubsection{The <Candidate $>$ Element}

For defining information about a candidate in a contest. <BallotSelection xsi:type $=$ "CandidateSelection" $>$ references $<$ Candidate $>$ elements to associate one or more candidates with a ballot selection. <Election > includes <Candidate>.

$<$ Candidate $>$ uses < PartyId $>$ to reference the candidate's political party. If the candidate is endorsed by other parties for a particular contest, the endorsing parties are referenced using the $<$ BallotSelection xsi:type="Candidate" $>$ sub-element (see section 4.2.2.2).

$<$ ExternalIdentifiers $>$ can be used to associate an ID with the candidate. If the type is not listed in enumeration <IdentifierType>, use other and include the type (that is not listed in the enumeration) in OtherType.

Table 4.10 - Attributes for <Candidate>

\begin{tabular}{|c|c|c|l|}
\hline Attribute & Required & Type & Attribute Description \\
\hline objectld & yes & xsd:ID & $\begin{array}{l}\text { Unique internal } \\
\text { identifier used by other } \\
\text { element(s) to reference } \\
\text { this element. }\end{array}$ \\
\hline
\end{tabular}

Table 4.11 - Elements for <Candidate>

\begin{tabular}{|c|c|c|c|}
\hline Element & Multiplicity & Tуре & $\begin{array}{c}\text { Element } \\
\text { Description }\end{array}$ \\
\hline$<$ BallotName $>$ & 1 & InternationalizedText & $\begin{array}{l}\text { For the candidate's } \\
\text { name as listed on } \\
\text { the ballot. }\end{array}$ \\
\hline$<$ ExternalIdentifiers $>$ & 0 or 1 & ExternalIdentifiers & $\begin{array}{l}\text { For associating } \\
\text { codes with the } \\
\text { candidate. }\end{array}$ \\
\hline$<$ FileDate $>$ & 0 or 1 & xsd:date & $\begin{array}{l}\text { Date when the } \\
\text { candidate filed for } \\
\text { the contest. }\end{array}$ \\
\hline$<$ IsIncumbent $>$ & 0 or 1 & xsd:boolean & $\begin{array}{l}\text { Boolean to indicate } \\
\text { whether the } \\
\text { candidate is the } \\
\text { incumbent for the } \\
\text { office associated } \\
\text { with the contest. } \\
\text { Assumed to be "no" } \\
\text { if not present. }\end{array}$ \\
\hline < IsTopTicket> & 0 or 1 & xsd:boolean & Boolean to indicate \\
\hline
\end{tabular}




\begin{tabular}{|c|c|c|c|}
\hline Element & Multiplicity & Tyре & $\begin{array}{c}\text { Element } \\
\text { Description }\end{array}$ \\
\hline & & & $\begin{array}{l}\text { whether the } \\
\text { candidate is the top } \\
\text { of a ticket that } \\
\text { includes multiple } \\
\text { candidates. } \\
\text { Assumed to be "no" } \\
\text { if not present. }\end{array}$ \\
\hline$<$ PartyId $>$ & 0 or 1 & xsd:IDREF & $\begin{array}{l}\text { Unique identifier for } \\
\text { a }<\text { Party > element. } \\
\text { For associating a } \\
\text { party with the } \\
\text { candidate. }\end{array}$ \\
\hline$<$ PersonId $>$ & 0 or 1 & xsd:IDREF & $\begin{array}{l}\text { Unique identifier for } \\
\text { a }<\text { Person }>\text { element. } \\
\text { For associating } \\
\text { more detailed } \\
\text { information about } \\
\text { the candidate. }\end{array}$ \\
\hline$<$ PostElectionStatus $>$ & 0 or 1 & CandidatePostElectionStatus & $\begin{array}{l}\text { Final status of the } \\
\text { candidate, e.g., } \\
\text { winner, withdrawn, } \\
\text { etc. }\end{array}$ \\
\hline$<$ PreElectionStatus $>$ & 0 or 1 & CandidatePreElectionStatus & $\begin{array}{l}\text { Registration status } \\
\text { of the candidate, } \\
\text { e.g., filed, qualified, } \\
\text { etc. }\end{array}$ \\
\hline
\end{tabular}

\section{Schema definition:}

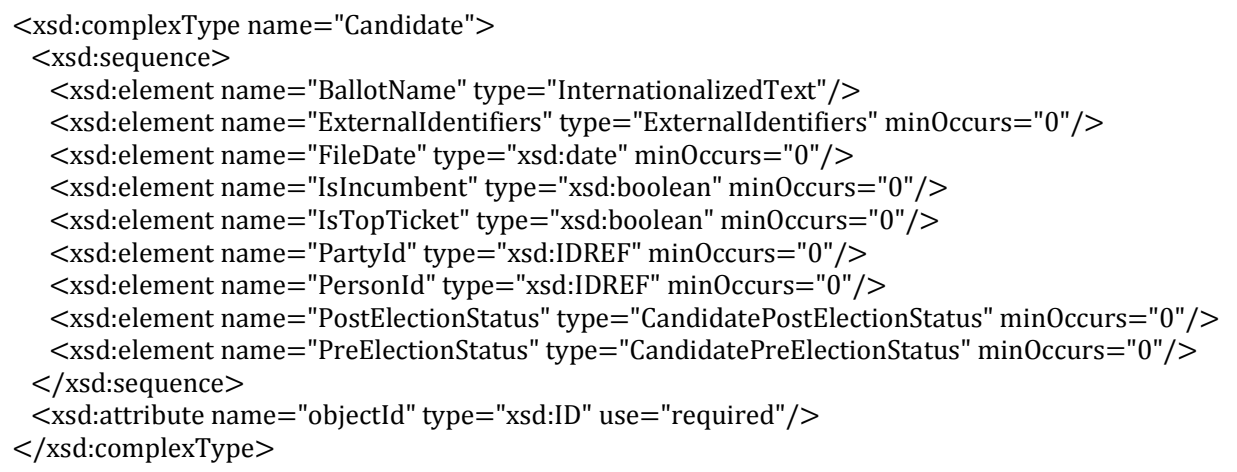




\subsubsection{The <ContactInformation> Element}

For defining contact information about objects such as persons, boards of authorities, organizations, etc. <Election $>$, <ElectionAdministration $>,<$ Person $>$, $<$ GpUnit $>$, and $<$ Office $>$ include $<$ ContactInformation $>$.

To include an address for the contact, use multiple occurrences of <AddressLine $>$. It is expected that the generating application will list the name of the person/organization in the first occurrence of <AddressLine $>$, with subsequent ordered occurrences for street address, city, state, zip code, etc. < Directions $>$ can be used to supply any additional address-related information that may appear in multiple languages.

$<$ ContactInformation $>$ includes < LatLng $>$ so as to associate latitude/longitude with the contact address.

$<$ Email $>,<$ Fax $>$, and $<$ Phone $>$ are of type AnnotatedString, which permits up to a 16-character annotation to be associated with the data, for example:

$<$ Email annotation="Work" $>$ john.a.smith@26003.org $</$ Email $>$

$<$ Email annotation="Home" $>$ john.a.smith@mybusiness.com $</$ Email $>$

Table 4.12 - Attributes for <ContactInformation>

\begin{tabular}{|c|c|c|c|}
\hline Attribute & Required & Type & Attribute Description \\
\hline label & no & xsd:string & For use as needed and compatibility with the VIP schema [6]. \\
\hline
\end{tabular}

Table 4.13 - Elements for <ContactInformation>

\begin{tabular}{|c|c|c|l|}
\hline Element & Multiplicity & \multicolumn{1}{|c|}{ Type } & \multicolumn{1}{|c|}{ Element Description } \\
\hline$<$ AddressLine $>$ & 0 or more & xsd:string & $\begin{array}{l}\text { For associating an address with the } \\
\text { contact. }\end{array}$ \\
\hline$<$ Directions $>$ & 0 or 1 & InternationalizedText & $\begin{array}{l}\text { Directional information in addition } \\
\text { to address information. }\end{array}$ \\
\hline$<$ Email $>$ & 0 or more & AnnotatedString & $\begin{array}{l}\text { Email address associated with the } \\
\text { contact. }\end{array}$ \\
\hline$<$ Fax $>$ & 0 or more & AnnotatedString & $\begin{array}{l}\text { Fax number associated with the } \\
\text { contact. }\end{array}$ \\
\hline$<$ LatLng $>$ & 0 or 1 & & $\begin{array}{l}\text { For latitude and longitude } \\
\text { information associated with the } \\
\text { contact. }\end{array}$ \\
\hline$<$ Name $>$ & 0 or 1 & xsd:string & Name associated with the contact. \\
\hline$<$ Phone $>$ & 0 or more & AnnotatedString & $\begin{array}{l}\text { Phone number associated with the } \\
\text { contact. }\end{array}$ \\
\hline
\end{tabular}




\begin{tabular}{|c|c|c|l|}
\hline Element & Multiplicity & Type & \multicolumn{1}{|c|}{ Element Description } \\
\hline$<$ Schedule $>$ & 0 or more & Schedule & $\begin{array}{l}\text { For associating a schedule with the } \\
\text { contact. }\end{array}$ \\
\hline$<$ Uri $>$ & 0 or more & xsd:anyURI & URI associated with the contact. \\
\hline
\end{tabular}

Table 4.14 - Attributes for <LatLng>

\begin{tabular}{|c|c|c|c|}
\hline Attribute & Required & Type & Attribute Description \\
\hline label & no & xsd:string & For use as needed and compatibility with the VIP schema. \\
\hline
\end{tabular}

Table 4.15 - Elements for <LatLng>

\begin{tabular}{|c|c|c|l|}
\hline Element & Multiplicity & Type & \multicolumn{1}{|c|}{ Element Description } \\
\hline <Latitude $>$ & 1 & xsd:float & Latitude of the contact location. \\
\hline$<$ Longitude $>$ & 1 & xsd:float & Longitude of the contact location. \\
\hline <Source $>$ & 0 or 1 & xsd:string & $\begin{array}{l}\text { System used to perform the lookup from location } \\
\text { name to lat/lng, e.g., the name of a geocoding } \\
\text { service. }\end{array}$ \\
\hline
\end{tabular}

\section{Schema definition:}

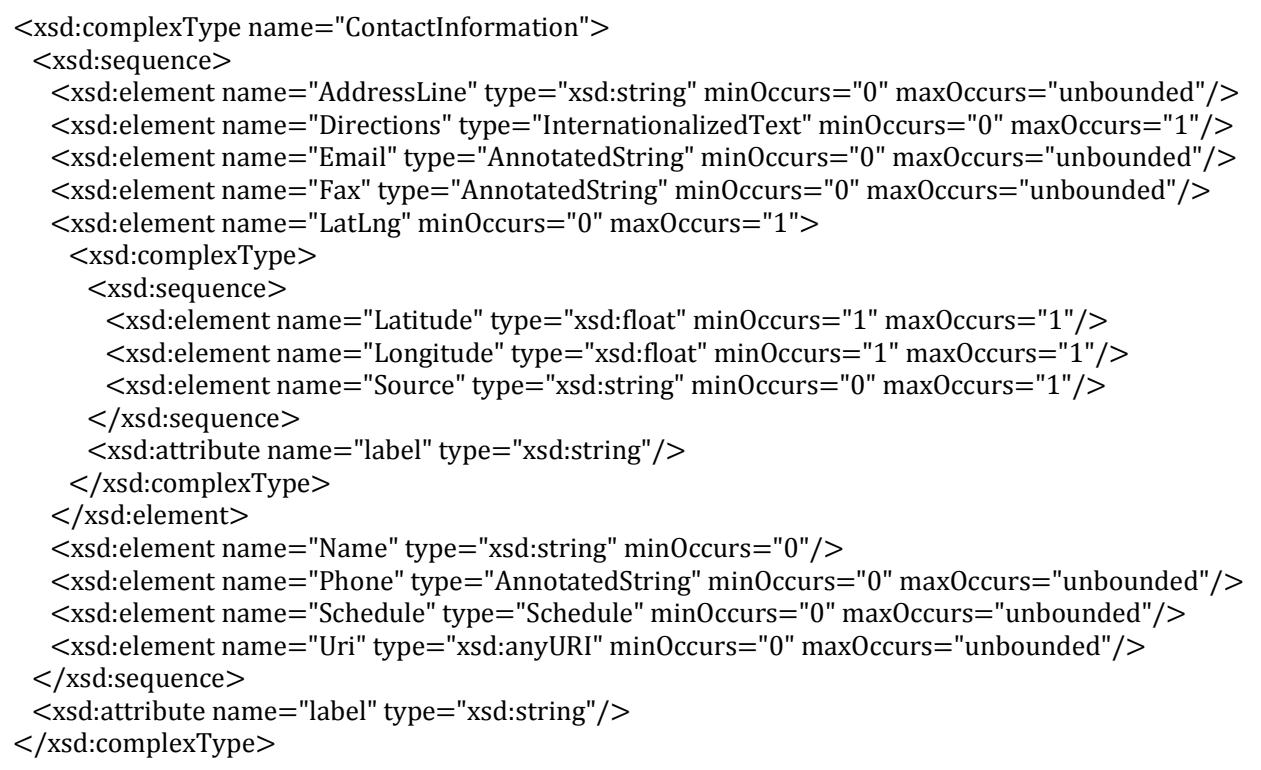




\subsubsection{The <Contest $>$ Element and Extension Base}

For defining a contest and linking the contest to the associated candidates, ballot measures, parties, or retention contests. <Election $>$ includes $<$ Contest $>$.

$<$ Contest $>$ is an abstract element with four xsi:types that get used according to the type of contest:

- $\quad<$ Contest xsi:type="BallotMeasureContest" $>$, used for a contest involving a ballot measure (see section 4.2.6.1)

- $\quad<$ Contest xsi:type="CandidateContest" $>$, used for a contest involving one or more candidates for an office (see section 4.2.6.2)

- $<$ Contest xsi:type="PartyContest" $>$, used for a contest for a straight party selection on the ballot (see section 0)

- $\quad<$ Contest xsi:type="RetentionContest">, used for a judicial or other type of retention contest (see section 4.2.6.4)

Each xsi:type is used to include information for that specified type of contest, for example, $<$ Contest xsi:type $=$ "CandidateContest" $>$ is used to include information about a candidate contest including the associated office and the number of candidates that can be voted for.

$<$ Contest $>$ includes <BallotSelection $>$ to link the selections on the ballot to the contest, e.g., to link one or more candidates to a candidate contest. Like <Contest $>,<$ BallotSelection $>$ is also an abstract element and has xsi types that essentially correspond to those of < <ontest $>$, as follows:

- $\quad<$ Contest xsi:type="BallotMeasureContest" $>$ includes <BallotSelection xsi:type $=$ "BallotMeasureSelection" $>$ (see section 4.2.2.1)

- <Contest xsi:type="CandidateContest" $>$ includes <BallotSelection xsi:type="CandidateSelection"> (see section 4.2.2.2)

- $\quad<$ Contest $x$ si:type="PartyContest" $>$ includes $<$ BallotSelection $x$ si:type="PartySelection" $>$ (see section 4.2.2.3)

- $\quad<$ Contest xsi:type="RetentionContest" $>$ includes <BallotSelection xsi:type="BallotMeasureSelection" $>$ (see section 4.2.2.1)

$<$ Contest $>$ includes a required <ElectoralDistrictId > reference to a <GpUnit $>$ defined for the geographical scope of the contest. For example, in a state senate contest, <ElectoralDistrictId $>$ would reference a <GpUnit xsi:type="ReportingUnit" $>$ element defined for the district associated with the contest. < Office $>$ also includes an optional reference that serves the same purpose. Note that for contests that are state-wide or county-wide and so forth, the same < GpUnit> defined for the state or county, etc., can be re-used.

$<$ Contest $>$ includes $<$ SummaryCounts $>$ for providing a summary of miscellaneous counts associated with the contest, including total number of ballots cast containing the contest, total number of overvotes, undervotes, or write-ins. The summary counts can be associated with the contest as a whole, or with precincts or other lower-level reporting units by using multiple occurrences of < SummaryCounts $>$ (see sections 4.2.8 and 4.2.23). 
$<$ SequenceOrder $>$ is used for results display ordering, i.e., to display contests according to a particular ordering. For example, "100” may indicate a U.S. Senatorial contest, “200” may indicate a U.S. Congressional contest, etc. <SequenceOrder $>$ is not appropriate to use as the contest order on the ballot; contest order on each ballot can be preserved, however, using the $<$ BallotStyle > element, which associates ballot styles with their corresponding precincts or other geopolitical units (see section 4.2.3).

When including <ExternalIdentifiers $>$, if the type is not listed in enumeration <IdentifierType $>$, use other and include the type (that is not listed in the enumeration) in <otherType $>$.

Table 4.16 - Attributes for <Contest>

\begin{tabular}{|c|c|c|l|}
\hline Attribute & Required & Type & \multicolumn{1}{|c|}{ Attribute Description } \\
\hline objectld & yes & xsd:ID & $\begin{array}{l}\text { Unique internal identifier used by other element(s) } \\
\text { to reference this element. }\end{array}$ \\
\hline
\end{tabular}

Table 4.17 - Elements for <Contest>

\begin{tabular}{|c|c|c|c|}
\hline Element & Multiplicity & Type & Element Description \\
\hline$<$ Abbreviation $>$ & 0 or 1 & xsd:string & Abbreviation for the contest. \\
\hline$<$ BallotSelection $>$ & 0 or more & BallotSelection & $\begin{array}{l}\text { For associating a ballot selection for } \\
\text { the contest, i.e., a candidate, a ballot } \\
\text { measure. }\end{array}$ \\
\hline$<$ BallotSubTitle $>$ & 0 or 1 & InternationalizedText & $\begin{array}{l}\text { Subtitle of the contest as it appears } \\
\text { on the ballot. }\end{array}$ \\
\hline$<$ BallotTitle $>$ & 0 or 1 & InternationalizedText & $\begin{array}{l}\text { Title of the contest as it appears on } \\
\text { the ballot. }\end{array}$ \\
\hline$<$ CountStatus $>$ & 0 or more & CountStatus & $\begin{array}{l}\text { For providing various counting status } \\
\text { associated with the contest. }\end{array}$ \\
\hline$<$ ElectoralDistrictId $>$ & 1 & xsd:IDREF & $\begin{array}{l}\text { Unique identifier for a <GpUnit> } \\
\text { element. For associating the contest } \\
\text { with a reporting unit that represents } \\
\text { the geographical scope of the contest, } \\
\text { e.g., a district, etc. }\end{array}$ \\
\hline$<$ ExternalIdentifiers $>$ & 0 or 1 & ExternalIdentifiers & $\begin{array}{l}\text { For associating an ID with the } \\
\text { contest. }\end{array}$ \\
\hline$<$ HasRotation $>$ & 0 or 1 & xsd:boolean & $\begin{array}{l}\text { Boolean to indicate whether the } \\
\text { selections in the contest are rotated. } \\
\text { Assumed to be "no" if not present. }\end{array}$ \\
\hline$<$ Name $>$ & 1 & xsd:string & $\begin{array}{l}\text { Name of the contest, not necessarily } \\
\text { as it appears on the ballot. }\end{array}$ \\
\hline
\end{tabular}




\begin{tabular}{|c|c|c|c|}
\hline Element & Multiplicity & Type & Element Description \\
\hline$<$ SequenceOrder $>$ & 0 or 1 & xsd:integer & $\begin{array}{l}\text { Orderering for listing the contest for } \\
\text { purposes of results display. If not } \\
\text { present, no order is assumed. }\end{array}$ \\
\hline$<$ SubUnitsReported $>$ & 0 or 1 & xsd:integer & $\begin{array}{l}\text { Number of subunits, e.g., precincts, } \\
\text { that have completed reporting votes } \\
\text { for this contest. }\end{array}$ \\
\hline$<$ SummaryCounts $>$ & 0 or more & SummaryCounts & $\begin{array}{l}\text { For providing a summary of } \\
\text { miscellaneous counts associated with } \\
\text { the contest. }\end{array}$ \\
\hline$<$ TotalSubUnits $>$ & 0 or 1 & xsd:integer & $\begin{array}{l}\text { Total number of subunits, e.g., } \\
\text { precincts that have this contest on the } \\
\text { ballot. }\end{array}$ \\
\hline$<$ VoteVariation $>$ & 0 or 1 & VoteVariation & $\begin{array}{l}\text { Vote variation associated with the } \\
\text { contest, e.g., } n \text {-of-m. }\end{array}$ \\
\hline$<$ OtherVoteVariation $>$ & 0 or 1 & xsd:string & For use when $<$ VoteVariation $>$ is other. \\
\hline
\end{tabular}

\section{Schema Definition:}

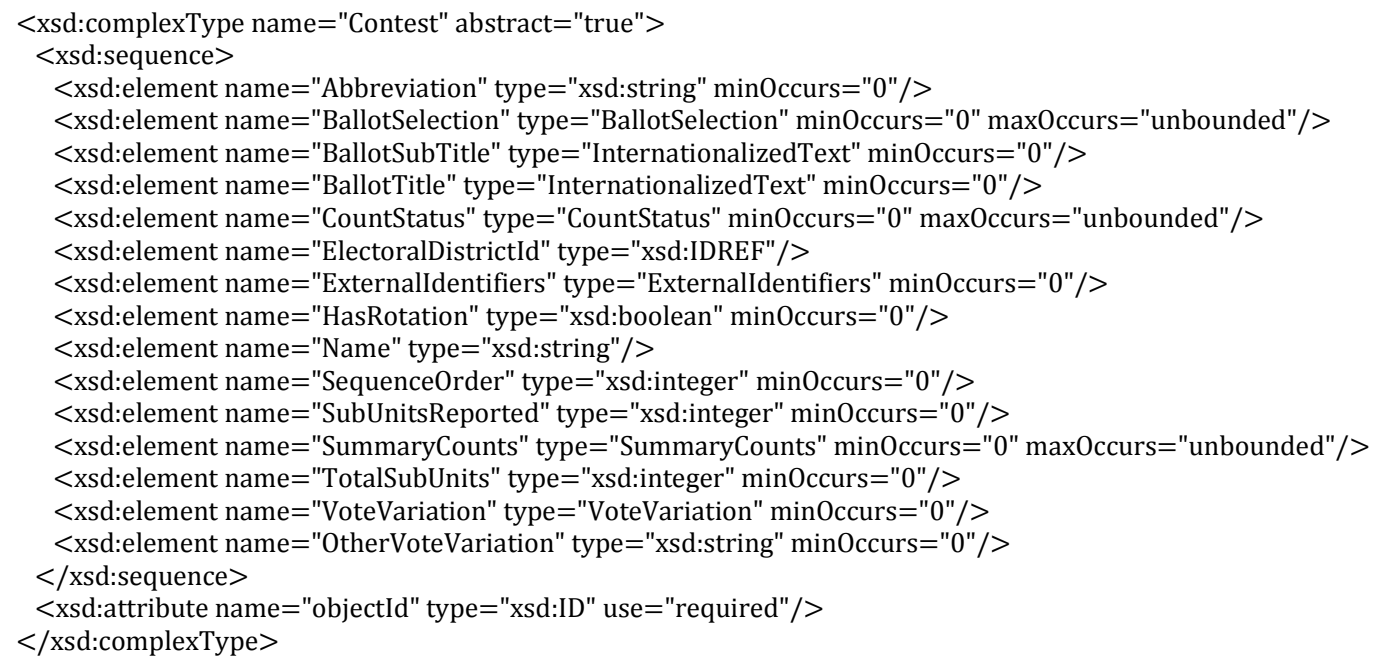




\subsubsection{The xsi:type=BallotMeasureContest}

For ballot measure (i.e., referenda or a tax measure) and judicial retention contests. It inherits the attributes and elements of < Contest> (see Table 4.16 and Table 4.17). Its syntax is:

$<$ Contest xsi:type="BallotMeasureContest" $\ldots />$

If the type of ballot measure is not listed in enumeration <BallotMeasureType $>$, use other and include the type (that is not listed in the enumeration) in <OtherType $>$.

Table 4.18 - Elements for <Contest xsi:type="BallotMeasureContest">

\begin{tabular}{|c|c|l|l|}
\hline Element & Multiplicity & \multicolumn{1}{|c|}{ Type } & \multicolumn{1}{|c|}{ Element Description } \\
\hline$<$ ConStatement $>$ & 0 or 1 & InternationalizedText & $\begin{array}{l}\text { For a statement on the ballot associated } \\
\text { with a “no" vote. }\end{array}$ \\
\hline$<$ EffectOfAbstain $>$ & 0 or 1 & InternationalizedText & $\begin{array}{l}\text { For a statement on the ballot detailing the } \\
\text { effect of abstaining from voting on the } \\
\text { ballot measure. }\end{array}$ \\
\hline$<$ FullText $>$ & 0 or 1 & InternationalizedText & $\begin{array}{l}\text { For full text on the ballot of the ballot } \\
\text { measure. }\end{array}$ \\
\hline$<$ InfoUri $>$ & 0 or 1 & xsd:anyURI & $\begin{array}{l}\text { For associating a URI with the ballot } \\
\text { measure contest. }\end{array}$ \\
\hline$<$ PassageThreshold $>$ & 0 or 1 & InternationalizedText & $\begin{array}{l}\text { For a statement on the ballot of the number } \\
\text { or percentage of votes needed to approve or } \\
\text { pass the ballot measure. }\end{array}$ \\
\hline$<$ ProStatement $>$ & 0 or 1 & InternationalizedText & $\begin{array}{l}\text { For a statement on the ballot associated } \\
\text { with a “yes” vote. }\end{array}$ \\
\hline$<$ SummaryText> & 0 or 1 & InternationalizedText & $\begin{array}{l}\text { For a summary on the ballot of the ballot } \\
\text { measure. }\end{array}$ \\
\hline$<$ Type $>$ & 0 or 1 & BallotMeasureType & For indicating the type of ballot measure. \\
\hline$<$ OtherType $>$ & 0 or 1 & xsd:string & Used when BallotMeasureType is other. \\
\hline
\end{tabular}

\section{Schema Definition:}

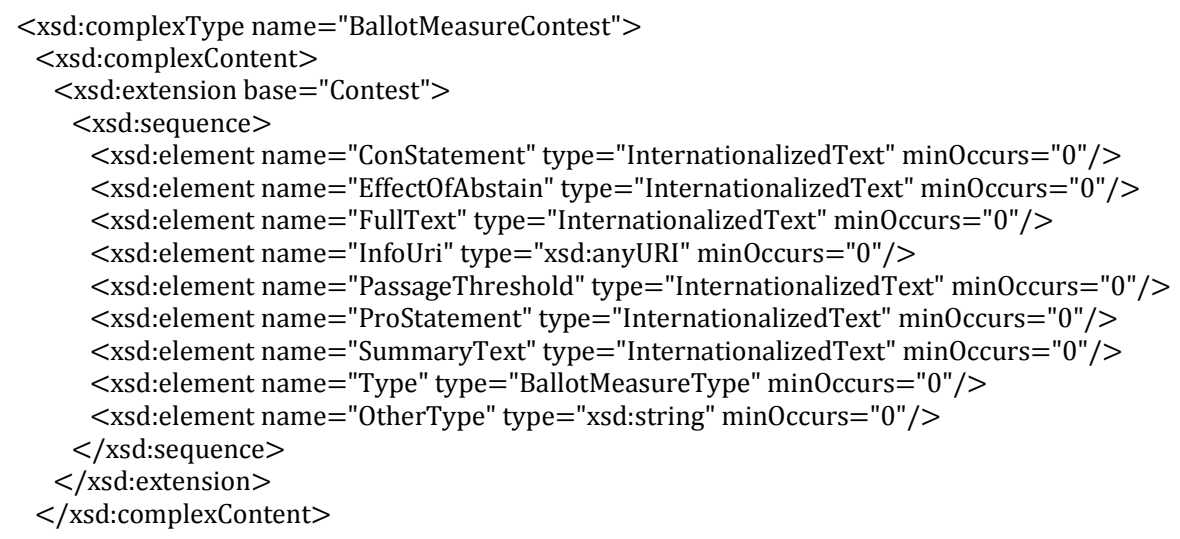


$</$ xsd:complexType $>$ 


\subsubsection{The $x$ si:type=CandidateContest}

For a contest that involves selecting one or more candidates. It inherits the attributes and elements of < Contest> (see Table 4.16 and Table 4.17). Its syntax is:

$<$ Contest xsi:type="CandidateContest" $\ldots />$

This element optionally references $<$ office $>$ and $<$ Party $>$. If the candidate contest is associated with a ticket (of candidates) and each candidate in the ticket is associated with a separate office, $<$ officeIds $>$ can contain reference to each of the separate offices. For example, if the contest is for the state governor ticket but Governor and Lieutenant (Lt.) Governor are both separate offices, $<$ OfficeIds $>$ will contain a reference first to the $<$ office $>$ element defined for the Governor's office and then to the Lt. Governor's office. In this case, it is expected that the generating application will list the multiple references according to a jurisdiction-defined ordering scheme, e.g., Governor first and Lt. Governor second.

Note that when using the <BallotSelection xsi:type="CandidateSelection" ${ }^{. . .} />$element to associate the candidates with a ballot selection for the contest (see section 4.2.2.2), the order of the candidates should match the order of offices. Again, using the example of the state governor ticket, if the offices are listed with Governor first and Lt. Governor second, then the order of the candidates in the <BallotSelection> element should be identical, with the Governor candidate first and the Lt. Governor candidate second.

Table 4.19 - Elements for <Contest xsi:type="CandidateContest">

\begin{tabular}{|c|c|c|l|}
\hline Element & Multiplicity & Type & \multicolumn{1}{|c|}{ Element Description } \\
\hline$<$ NumberElected $>$ & 0 or 1 & xsd:integer & $\begin{array}{l}\text { Number of candidates that are elected in the contest } \\
\text { (“n” of n-of-m). }\end{array}$ \\
\hline$<$ OfficeIds> & 0 or 1 & xsd:IDREFS & $\begin{array}{l}\text { Unique identifier for one or more <Office }>\text { elements. } \\
\text { For associating office descriptions. }\end{array}$ \\
\hline$<$ PrimaryPartylds> & 0 or 1 & xsd:IDREFS & $\begin{array}{l}\text { Unique identifier for one or more <Party }>\text { elements. } \\
\text { For associating parties with the contest. }\end{array}$ \\
\hline$<$ VotesAllowed $>$ & 1 & xsd:integer & $\begin{array}{l}\text { Maximum number of votes/write-ins per voter in this } \\
\text { contest. }\end{array}$ \\
\hline
\end{tabular}

Schema Definition:

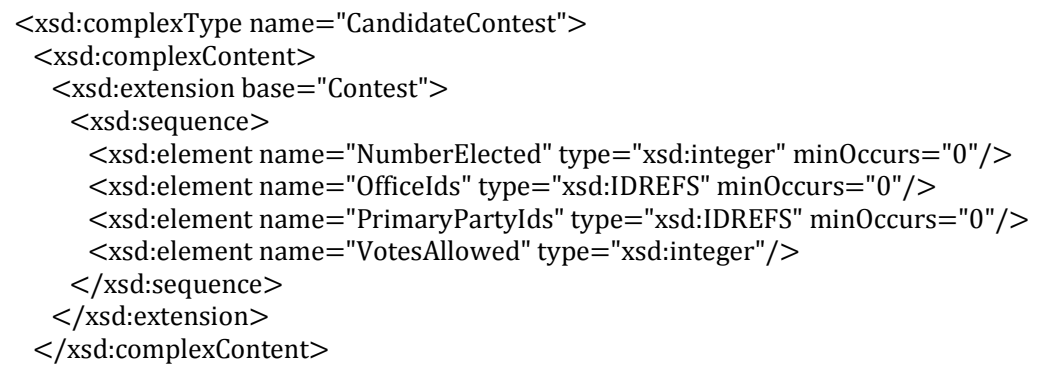


$</$ xsd:complexType $>$ 


\subsubsection{The $x$ si:type=PartyContest}

For a contest that involves choosing a party, typically for a straight party selection on the ballot. Its syntax is:

$<$ Contest xsi:type $={ }^{\text {"PartyContest }}{ }^{\prime} . . . />$

Schema definition:

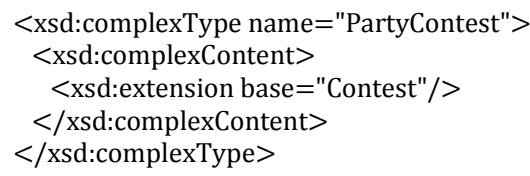




\subsubsection{The $x$ si:type=RetentionContest}

For judicial retention or other types of retention contests. Retention contests can be treated essentially as ballot measure contests, however this element differs from <Contest xsi:type $=$ "BallotMeasureContest" $>$ in that it can include a reference to a candidate or the associated office.

This element uses < BallotMeasureContest $>$ as an extension base. Therefore, it inherits the attributes and elements of <Contest $>$ as well as $<$ BallotMeasureContest $>$ (see Table 4.16 and Table 4.17 for <Contest $>$ and Table 4.18 for <Contest xsi:type="BallotMeasure" $>$ ). Its syntax is:

$<$ Contest xsi:type="RetentionContest" $\ldots />$

Table 4.20 - Elements for <Contest xsi:type="RetentionContest">

\begin{tabular}{|c|c|c|l|}
\hline Element & Multiplicity & Type & \multicolumn{1}{|c|}{ Element Description } \\
\hline$<$ CandidateId $>$ & 1 & xsd:IDREF & $\begin{array}{l}\text { Unique identifier for a <Candidate }>\text { element. For associating } \\
\text { a candidate with the retention contest. }\end{array}$ \\
\hline < OfficeId $>$ & 0 or 1 & xsd:IDREF & $\begin{array}{l}\text { Unique identifier for an <Office }>\text { element. For associating an } \\
\text { office description with the retention contest. }\end{array}$ \\
\hline
\end{tabular}

Schema Definition:

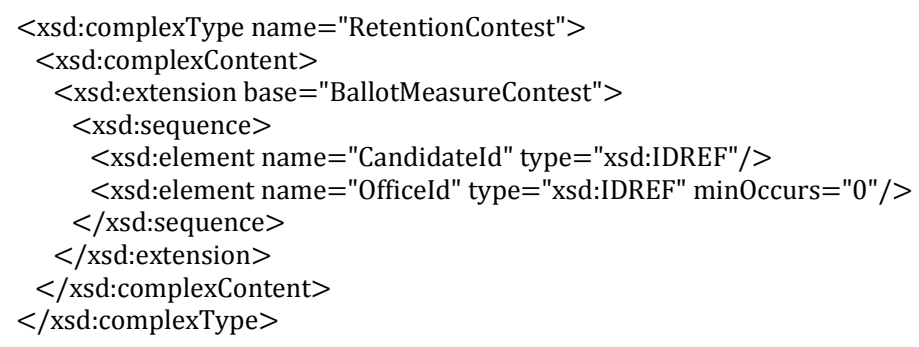




\subsubsection{The <CountStatus $>$ Element}

For reporting on the counting status for various items such as ballot types or write-ins, e.g., whether for a certain type of ballot, the counts are in progress, not yet started, complete, etc. $<$ Contest $>$, $<$ Election $>$, and $<$ GpUnit $>$ include $<$ CountStatus $>$.

If the type of count item is not listed in enumeration <CountItemType>, use other and include the type (that is not listed in the enumeration) in <otherType $>$.

Table 4.21 - Elements for <CountStatus $>$

\begin{tabular}{|c|c|c|l|}
\hline Element & Multiplicity & Type & \multicolumn{1}{|c|}{ Element Description } \\
\hline$<$ Status $>$ & 1 & CountItemStatus & $\begin{array}{l}\text { The status of the count, from the <CountItemStatus }> \\
\text { enumeration. }\end{array}$ \\
\hline$<$ Type $>$ & 1 & CountItemType & $\begin{array}{l}\text { The type of item, from the <CountItemType }> \\
\text { enumeration. }\end{array}$ \\
\hline$<$ OtherType $>$ & 0 or 1 & xsd:string & Used when <Type $>$ is other. \\
\hline
\end{tabular}

\section{Schema Definition:}

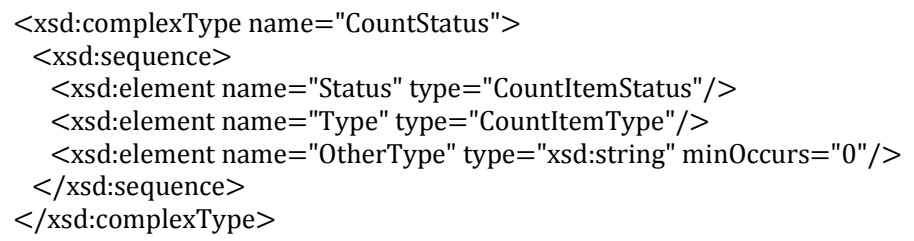




\subsubsection{The <Counts> Extension Base Element}

$<$ Counts $>$ is not used or referenced directly; it is used only as an extension base for the following elements:

- $\quad<$ SummaryCounts $>$, for reporting on contest and geopolitical unit summary vote counts (see section 4.2.23); and

- $\quad<$ VoteCounts $>$, for reporting on contest vote counts (see section 4.2.24).

These elements inherit the attributes and elements of $<$ Counts $>$.

$<$ Counts $>$ provides the capability to filter vote counts by device characteristics or by various types of ballots or write-ins, or to link the counts to a <GpUnit> element defined for, e.g., a precinct.

The <IsSuppressedForPrivacy > boolean is used for cases when a jurisdiction has a requirement for suppressing a contest's vote count when associated with a precinct and the count is so small as to potentially show how specific voters at the precinct's polling place may have voted (typically under 10 votes). This is also a potential concern when filtering on device type or ballot type for a precinct; the accessible voting device will typically have relatively low counts. In some cases, jurisdictions suppress the votes for that contest/precinct/device/ballot type combination, but add them back to the contest totals, thus meeting the requirement for voter privacy. However, this results in the precinct counts not adding up to the aggregated totals, thus this boolean can be used to indicate which of the counts were suppressed.

If the type of count item is not listed in enumeration <CountItemType>, use other and include the type (that is not listed in the enumeration) in <otherType >.

Table 4.22 - Elements for <Counts $>$

\begin{tabular}{|c|c|c|l|}
\hline Element & Multiplicity & \multicolumn{1}{|c|}{ Type } & \multicolumn{1}{|c|}{ Element Description } \\
\hline <Device $>$ & 0 or 1 & Device & For filtering counts by device type. \\
\hline <GpUnitId $>$ & 0 or 1 & xsd:IDREF & $\begin{array}{l}\text { Unique identifier for a <GpUnit> element. For } \\
\text { associating counts with a geopolitical unit, } \\
\text { e.g., a precinct, a county, a township, etc. }\end{array}$ \\
\hline <IsSuppressedForPrivacy> & 0 or 1 & xsd:boolean & $\begin{array}{l}\text { Boolean to indicate if votes are suppressed for } \\
\text { voter privacy, e.g., true or false. Assumed to be } \\
\text { false if not present. }\end{array}$ \\
\hline$<$ Type> & 0 or 1 & CountltemType & $\begin{array}{l}\text { The type of count being used as a filter on the } \\
\text { vote counts, e.g., election day, early voting, } \\
\text { etc. }\end{array}$ \\
\hline$<$ OtherType> & 0 or 1 & xsd:string & Used when <Type > is other. \\
\hline
\end{tabular}




\section{Schema Definition:}

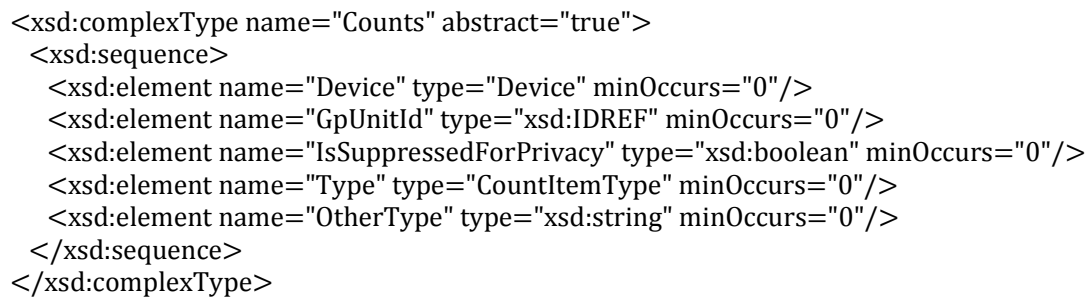

$</$ xsd:complexType $>$ 


\subsubsection{The <Device $>$ Element}

For filtering vote counts by various attributes associated with devices. <GpUnit> includes $<$ Device $>$, and $<$ SummaryCounts $>$ and $<$ VoteCounts $>$ include $<$ Device $>$ as a result of it being included from extension base $<$ Counts $>$.

If manual counting of ballots is being employed, use the < DeviceType > enumeration value manual-count.

\section{Table 4.23 - Elements for <Device>}

\begin{tabular}{|c|c|c|l|}
\hline Element & Multiplicity & Type & \multicolumn{1}{|c|}{ Element Description } \\
\hline <Manufacturer $>$ & 0 or 1 & xsd:string & Manufacturer of the device. \\
\hline <Model> & 0 or 1 & xsd:string & $\begin{array}{l}\text { Manufacturer's device model, used to filter on, e.g., a } \\
\text { specific model of DRE or other device type. }\end{array}$ \\
\hline$<$ Type $>$ & 0 or 1 & DeviceType & Enumerated type of device, e.g., DRE, opscan-precinct, etc. \\
\hline$<$ OtherType $>$ & 0 or 1 & xsd:string & Used when <Type $>$ is other. \\
\hline
\end{tabular}

\section{Schema Definition:}

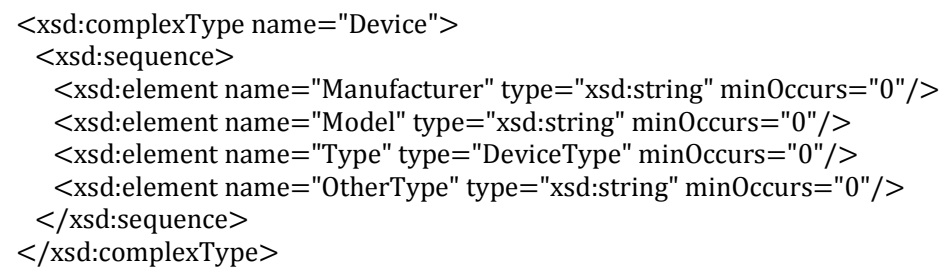




\subsubsection{The <Election> Element}

For defining the status of the election and associated information such as candidates, contests, and vote counts.

$<$ Election $>$ includes the major elements that are specific to an election: $<$ BallotStyle $>$, $<$ Candidate $>$, and < Contest $>$. < Election $>$ "wraps" occurrences of these elements in container elements for the purpose of making large instance files easier to manipulate in XML viewers and editors.

$<$ Election > includes a required < ElectionScopeId > reference to a < GpUnit> element for the purpose of identifying the geographical scope of the election. For example, for an election within a county, <ElectionScodeId $>$ would include a reference to a $<$ GpUnit $>$ defined for the county. If it is desired to include election authority information, the <GpUnit> can include $<$ ElectionAdministration $>$, see sections 4.2.14.2 and 0 .

Table 4.24 - Elements for <Election>

\begin{tabular}{|c|c|c|c|}
\hline Element & Multiplicity & Туре & Element Description \\
\hline$<$ BallotStyleCollection $>$ & 0 or 1 & & $\begin{array}{l}\text { Wrapper element for <BallotStyle> } \\
\text { definitions. }\end{array}$ \\
\hline$<$ BallotStyle $>$ & 1 or more & BallotStyle & $\begin{array}{l}\text { For defining ballot styles associated } \\
\text { with the election. }\end{array}$ \\
\hline$<$ CandidateCollection $>$ & 0 or 1 & & $\begin{array}{l}\text { Wrapper element for <Candidate> } \\
\text { definitions. }\end{array}$ \\
\hline$<$ Candidate $>$ & 1 or more & Candidate & $\begin{array}{l}\text { For defining candidates associated } \\
\text { with the election. }\end{array}$ \\
\hline$<$ ContactInformation $>$ & 0 or 1 & ContactInformation & $\begin{array}{l}\text { For associating various contact } \\
\text { information with the election. }\end{array}$ \\
\hline$<$ ContestCollection $>$ & 0 or 1 & & $\begin{array}{l}\text { Wrapper element for < Contest }> \\
\text { definitions. }\end{array}$ \\
\hline$<$ Contest $>$ & 1 or more & Contest & $\begin{array}{l}\text { For defining contests associated with } \\
\text { the election. }\end{array}$ \\
\hline$<$ CountStatus $>$ & 0 or more & CountStatus & $\begin{array}{l}\text { For providing various counting status } \\
\text { on types of ballots or other items. }\end{array}$ \\
\hline$<$ ElectionScopeId $>$ & 1 & xsd:IDREF & $\begin{array}{l}\text { Unique identifier for a <GpUnit> } \\
\text { element. For associating the election } \\
\text { with a reporting unit that represents } \\
\text { the geographical scope of the } \\
\text { election, e.g., a state, a county, etc. }\end{array}$ \\
\hline$<$ ExternalIdentifiers $>$ & 0 or 1 & ExternalIdentifiers & $\begin{array}{l}\text { For associating an ID with the } \\
\text { election. }\end{array}$ \\
\hline$<$ Name $>$ & 1 & InternationalizedText & For including a name for the election; \\
\hline
\end{tabular}




\begin{tabular}{|c|c|c|l|}
\hline Element & Multiplicity & Type & \multicolumn{1}{|c|}{ Element Description } \\
\hline <StartDate> & 1 & xsd:date & $\begin{array}{l}\text { Calendar start date of the election, } \\
\text { e.g., "2018-11-04". }\end{array}$ \\
\hline appears on the ballot.
\end{tabular}

\section{Schema Definition:}

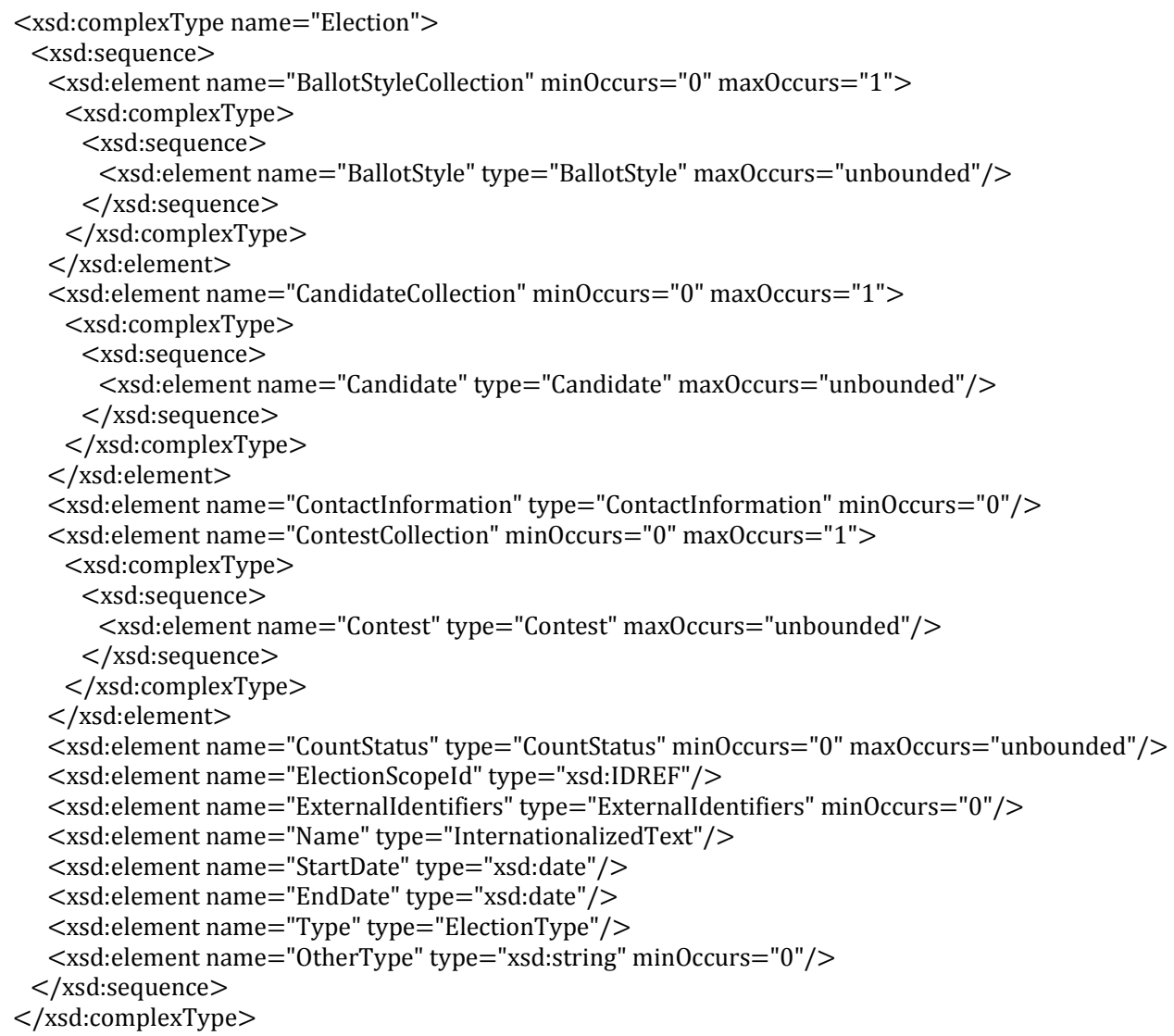




\subsubsection{The <ElectionAdministration> Element}

Used to provide various information about an election authority. $<$ GpUnit xsi:type="ReportingUnit" $>$ includes < ElectionAdministration $>$.

The <Election> element references the <GpUnit> defined as the scope of the election, e.g., if the scope of the election is for a county, <Election > references the <GpUnit $>$ element defined for that county. <ElectionAdministration > would presumably be included from that <GpUnit $>$ element defined as the scope of the election.

$<$ ElectionAdministration > includes <ContactInformation > for the election authority and, using $<$ ElectionOfficialPersonIds $>$, references one or more $<$ Person $>$ elements defined for individuals/organizations associated with the election authority.

Table 4.25 - Elements for <ElectionAdministration>

\begin{tabular}{|c|c|c|l|}
\hline Element & Multiplicity & Type & \multicolumn{1}{|c|}{ Element Description } \\
\hline <ContactInformation> & 0 or 1 & ContactInformation & For including various contact information. \\
\hline <ElectionOfficialPersonIds> & 0 or 1 & xsd:IDREFS & $\begin{array}{l}\text { Unique identifier for one or more } \\
<\text { Person }>\text { elements defined for the election } \\
\text { authority. }\end{array}$ \\
\hline <Name $>$ & 0 or 1 & xsd:string & Name of the election authority. \\
\hline
\end{tabular}

Schema Definition:

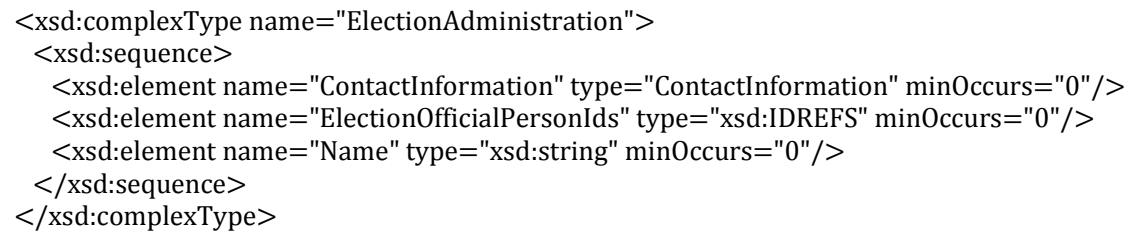




\subsubsection{The <ElectionReport> Element}

The root element; for defining items pertaining to the status and format of the report and when it was generated. The optional < signature > element is used for an XML digital signature [9]. $<$ Signature $>$ must be the last element of < ElectionReport $>$.

$<$ ElectionReport $>$ includes the major elements that are not necessarily specific to an election and that therefore can exist in a pre-election report: $\langle$ GpUnit $\rangle,\langle$ Office $\rangle$ and $\langle$ OfficeGroup $\rangle,\langle$ Party $\rangle$, $<$ Person $>$, and $<$ Election $>$. As with $<$ Election $>$, <ElectionReport $>$ "wraps" occurrences of the elements (excepting $<$ Election $>$ ) in container elements for the purpose of making the instance files easier to manipulate in XML viewers and editors.

When a particular ordering of items such as offices or political parties are important to preserve, it is expected that the generating application will define those elements according to any ordering scheme in place.

Table 4.26 - Elements for <ElectionReport>

\begin{tabular}{|c|c|c|c|}
\hline Element & Multiplicity & Туре & Element Description \\
\hline$<$ Election $>$ & 0 or more & Election & For associating elections with the report. \\
\hline$<$ ExternalIdentifiers $>$ & 0 or 1 & ExternalIdentifiers & For associating an ID with the report. \\
\hline$<$ Format $>$ & 1 & ReportDetailLevel & $\begin{array}{l}\text { Detail level of the report, e.g., contest } \\
\text { summary, precinct level results, etc. }\end{array}$ \\
\hline$<$ GeneratedDate $>$ & 1 & xsd:dateTime & $\begin{array}{l}\text { Identifies the date and time that the } \\
\text { election report was generated. }\end{array}$ \\
\hline$<$ GpUnitCollection $>$ & 0 or 1 & & Wrapper element for <GpUnit> definitions. \\
\hline$<$ GpUnit $>$ & 1 or more & GpUnit & $\begin{array}{l}\text { For associating geopolitical units with the } \\
\text { report. }\end{array}$ \\
\hline$<$ Issuer $>$ & 1 & xsd:string & Identification of the report issuer. \\
\hline$<$ IssuerAbbreviation $>$ & 1 & xsd:string & $\begin{array}{l}\text { An abbreviation of the report issuer such } \\
\text { as the 2-character U.S. Census Bureau } \\
\text { abbreviation of the state whose results are } \\
\text { being reported, e.g., AL, TX, MN, etc. }\end{array}$ \\
\hline$<$ IsTest $>$ & 0 or 1 & xsd:boolean & $\begin{array}{l}\text { Used to indicate whether the report is a } \\
\text { test report. Assumed to be "no" if not } \\
\text { present. }\end{array}$ \\
\hline$<$ Notes $>$ & 0 or 1 & xsd:string & $\begin{array}{l}\text { For including an arbitrary message with } \\
\text { the report. }\end{array}$ \\
\hline$<$ OfficeCollection $>$ & 0 or 1 & & Wrapper element for < office> definitions. \\
\hline$<$ Office $>$ & 1 or more & Office & For associating offices with the report. \\
\hline$<$ OfficeGroup $>$ & 0 or more & OfficeGroup & $\begin{array}{l}\text { For associating a name for a grouping of } \\
\text { offices with the report. }\end{array}$ \\
\hline
\end{tabular}




\begin{tabular}{|c|c|c|c|}
\hline Element & Multiplicity & Type & Element Description \\
\hline$<$ PartyCollection $>$ & 0 or 1 & & Wrapper element for < Party $>$ definitions. \\
\hline$<$ Party $>$ & 1 or more & Party & For associating parties with the report. \\
\hline$<$ PersonCollection $>$ & 0 or 1 & & Wrapper element for < Person > definitions. \\
\hline$<$ Person $>$ & 1 or more & Person & For associating persons with the report. \\
\hline$<$ SequenceStart $>$ & 1 & xsd:integer & $\begin{array}{l}\text { The report's number as part of a sequence } \\
\text { of reports, used with < }<\text { SequenceEnd }>\text { so as } \\
\text { to be read as, e.g., } 1 \text { of } 1,1 \text { of } 2,2 \text { of } 2 \text {, } \\
\text { etc. Starts with “1". }\end{array}$ \\
\hline$<$ SequenceEnd $>$ & 1 & xsd:integer & $\begin{array}{l}\text { The upper bound of the sequence; e.g., " } 1 \text { " } \\
\text { if there is only } 1 \text { report, " } 2 \text { " if there are } \\
\text { two reports in the sequence, etc. }\end{array}$ \\
\hline$<$ Status $>$ & 1 & ResultsStatus & $\begin{array}{l}\text { Status of the election report, e.g., test } \\
\text { mode, unofficial, etc. }\end{array}$ \\
\hline$<$ TestType $>$ & 0 or 1 & xsd:string & $\begin{array}{l}\text { A description of the type of test, e.g., pre- } \\
\text { election, logic and accuracy, etc. }\end{array}$ \\
\hline$<$ VendorApplicationID $>$ & 1 & xsd:string & $\begin{array}{l}\text { An identifier of the vendor application } \\
\text { generating the election report, e.g., X- } \\
\text { EMS version 3.1.a. }\end{array}$ \\
\hline$<$ Signature $>$ & 0 or 1 & Signature & $\begin{array}{l}\text { Reference to the <Signature }>\text { element of } \\
\text { the W3C digital signature schema } \\
\text { imported into this schema. }\end{array}$ \\
\hline
\end{tabular}

\section{Schema Definition:}

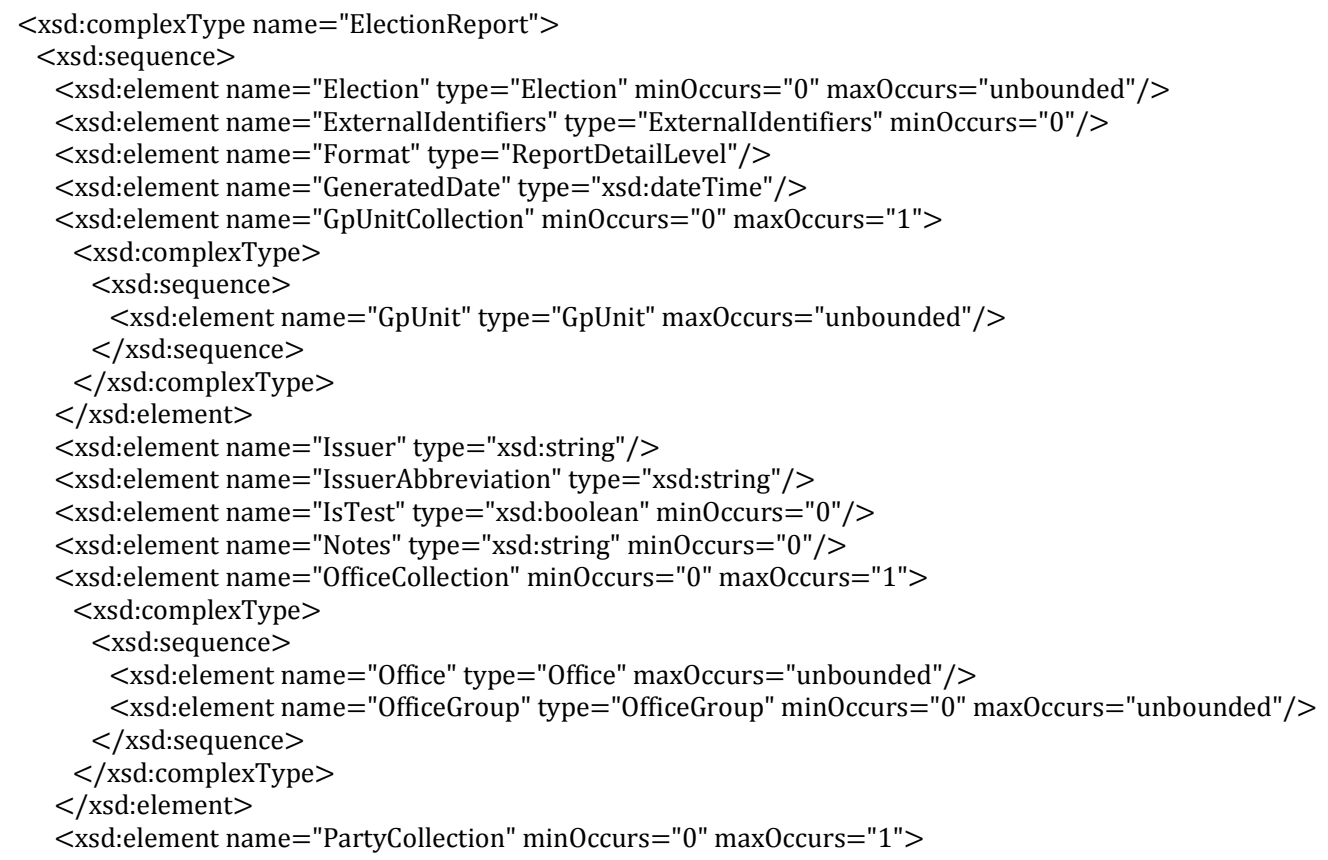




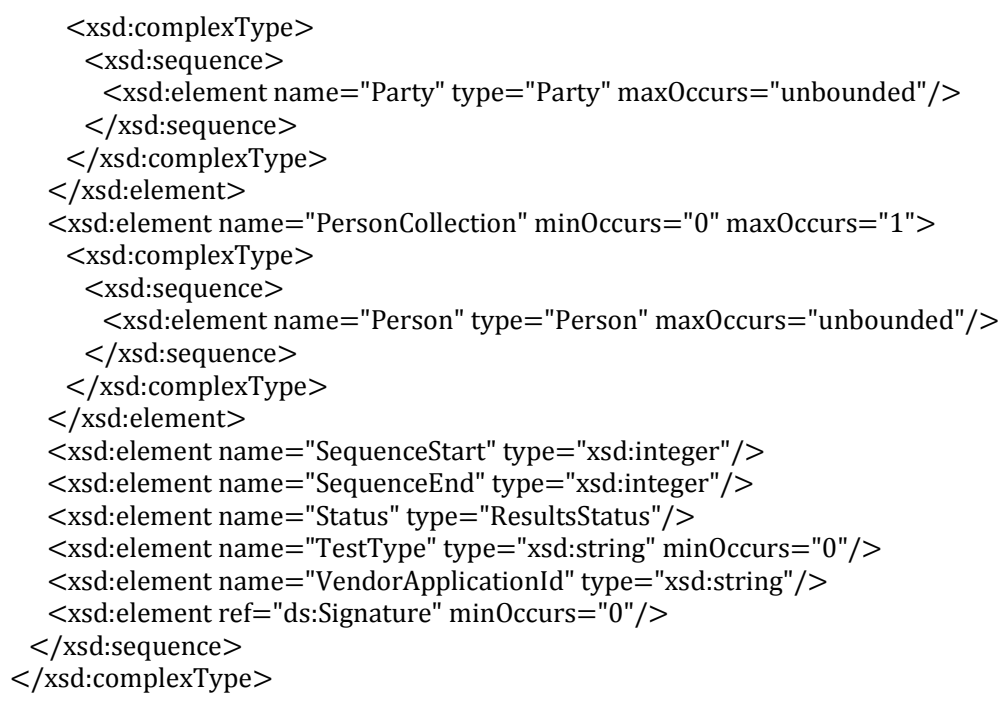




\subsubsection{The <Externalldentifiers> Element}

For associating a jurisdiction's codes, i.e., identifiers, with objects such as candidates, offices, or geopolitical units such as counties, towns, precincts, etc. ${ }^{3}$ Multiple occurrences of the $<$ ExternalIdentifier> sub-element can be used to associate multiple codes, e.g., if there is a desire to associate multiple codes with a particular object such as FIPS (Federal Information Processing Standard [10]) codes as well as OCD-IDs (Open Civic Data Identifiers [11]), as follows:

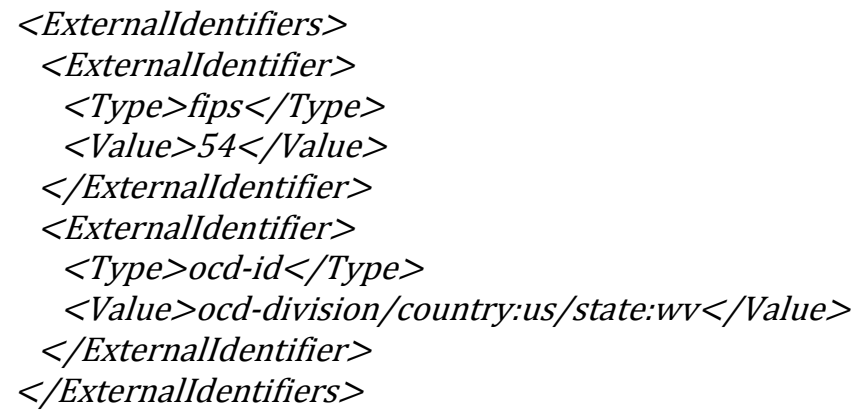

For elements that include <ExternalIdentifiers $>$, if the type is not listed in enumeration $<$ IdentifierType>, use other and include the type (that is not listed in the enumeration) in OtherType, e.g.,

$<$ ExternalIdentifier $>$

$<$ Type $>$ other $</$ Type $>$

$<$ Value $>101-A</$ Value $>$

$<$ OtherType $>$ Ohio County Precinct Numbers $</$ OtherType $>$

$</$ ExternalIdentifier $>$

Table 4.27 - Attributes for <Externalldentifier>

\begin{tabular}{|c|c|c|c|}
\hline Attribute & Required & Type & Attribute Description \\
\hline label & no & xsd:string & For use as needed and compatibility with the VIP schema. \\
\hline
\end{tabular}

Table 4.28 - Elements for <Externalldentifier>

\begin{tabular}{|c|c|c|l|}
\hline Element & Multiplicity & Type & \multicolumn{1}{|c|}{ Element Description } \\
\hline$<$ Type $>$ & 1 & IdentifierType & An identifier type, e.g., FIPS. \\
\hline$<$ OtherType $>$ & 0 or 1 & xsd:string & Used when $<$ IdentifierType $>$ value is other. \\
\hline$<$ Value $>$ & 1 & xsd:string & The identifier used by the jurisdiction. \\
\hline
\end{tabular}

Schema definition:

\footnotetext{
${ }^{3}$ A future version of this standard may reference a registry containing election data-related codes, thus this element may change.
} 


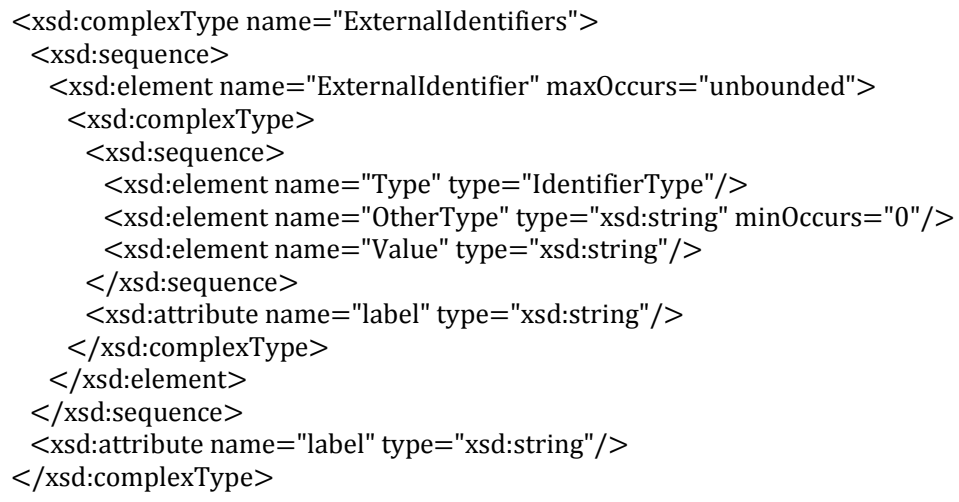




\subsubsection{The <GpUnit> Element and Extension Base}

For defining geopolitical units such as cities, districts, jurisdictions, precincts or split precincts, for the purpose of associating contests, offices, vote counts, or other information with the geographies. <ElectionReport> includes <GpUnit>.

$<$ GpUnit> is an abstract element with two xsi:types:

- $\quad<$ GpUnit xsi:type="ReportingDevice" $>$, for associating vote counts with a specific votecapture device (see section 4.2.14.1)

- $\quad<$ GpUnit xsi:type="ReportingUnit" $>$, for associating vote counts with geopolitical units such as cities, districts, counties, precincts, etc. (see section 4.2.14.2)

$<$ Election $>$ and $<$ Contest $>$ contain a required reference to a $<$ GpUnit $>$ element defined as the jurisdiction of the election or contest respectively; < office $>$ contains a similar reference that is optional. <SummaryCounts $>$ and $<$ VoteCounts $>$ reference $<$ GpUnit $>$ to link vote or summary counts to <GpUnit> elements defined for precincts or other types of geopolitical units. $<$ BallotStyle $>$ references < GpUnit $>$ to link a ballot style to its corresponding geopolitical unit.

$<$ GpUnit $>$ includes < SummaryCounts $>$ so as to provide summary counts for the corresponding geopolitical geography. <ComposingGpUnitIds > is used to reference other <GpUnit> elements and associate them with the parent <GpUnit>, with the rule that each referenced element must be defined for a geopolitical unit that is contained within the parent <GpUnit> element (see section 5.2.1).

When including < ExternalIdentifiers $>$, if the type is not listed in enumeration <IdentifierType $>$, use other and include the type (that is not listed in the enumeration) in <otherType $>$.

Table 4.29 - Attributes for $<$ GpUnit $>$

\begin{tabular}{|c|c|c|l|}
\hline Attribute & Required & Type & \multicolumn{1}{|c|}{ Attribute Description } \\
\hline objectId & yes & xsd:ID & $\begin{array}{l}\text { Unique internal identifier used by other element(s) to reference } \\
\text { this element. }\end{array}$ \\
\hline
\end{tabular}

Table 4.30 - Elements for <GpUnit>

\begin{tabular}{|c|c|c|l|}
\hline Element & Multiplicity & Type & \multicolumn{1}{|c|}{ Element Description } \\
\hline <ComposingGpUnitIds> & 0 or 1 & xsd:IDREFS & $\begin{array}{l}\text { Unique identifier for one or more } \\
<G p U n i t>\text { elements. For creating a } \\
\text { reference to another <GpUnit }>\text { that is } \\
\text { contained with the parent <GpUnit }>\end{array}$ \\
\hline <ExternalIdentifiers> & 0 or 1 & ExternalIdentifiers & $\begin{array}{l}\text { For associating an ID with the <GpUnit }>, \\
\text { e.g., a district’s or county’s code. }\end{array}$ \\
\hline
\end{tabular}




\begin{tabular}{|c|c|c|l|}
\hline Element & Multiplicity & \multicolumn{1}{|c|}{ Type } & \multicolumn{1}{|c|}{ Element Description } \\
\hline$<$ Name $>$ & 0 or 1 & xsd:string & Name of the geopolitical unit. \\
\hline$<$ SummaryCounts $>$ & 0 or more & SummaryCounts & $\begin{array}{l}\text { Ballot summary counts (overvotes, } \\
\text { undervotes, total ballots, etc.) optionally } \\
\text { broken down by device type and ballot } \\
\text { class. }\end{array}$ \\
\hline
\end{tabular}

\section{Schema Definition:}

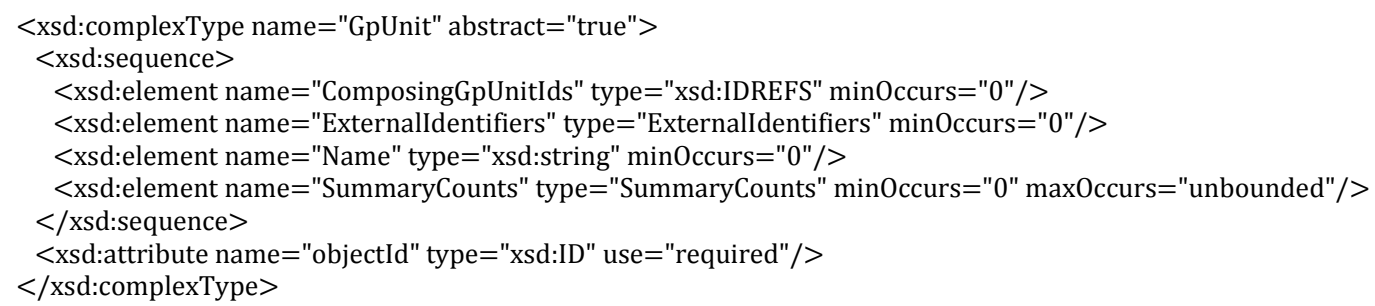




\subsubsection{The xsi:type=ReportingDevice}

For reporting counts associated with a specific vote-capture device. It inherits the attributes and elements of <GpUnit> (see Table 4.29 and Table 4.30). Its syntax is:

$<$ GpUnit xsi:type $="$ ReportingDevice"...$/>$

It identifies a specific vote-capture device by using the < SerialNumber $>$ element, and then includes $<$ Device $>$ to identify other characteristics of the device such as manufacturer and model.

Table 4.31 - Elements for <GpUnit xsi:type="ReportingDevice">

\begin{tabular}{|c|c|c|l|}
\hline Element & Multiplicity & Type & \multicolumn{1}{|c|}{ Element Description } \\
\hline$<$ Device $>$ & 0 or 1 & Device & $\begin{array}{l}\text { For filtering the vote counts by device type, model, and } \\
\text { manufacturer. }\end{array}$ \\
\hline$<$ SerialNumber $>$ & 0 or 1 & xsd:string & $\begin{array}{l}\text { A serial number or otherwise identifier associated with the } \\
\text { device. }\end{array}$ \\
\hline
\end{tabular}

Schema Definition:

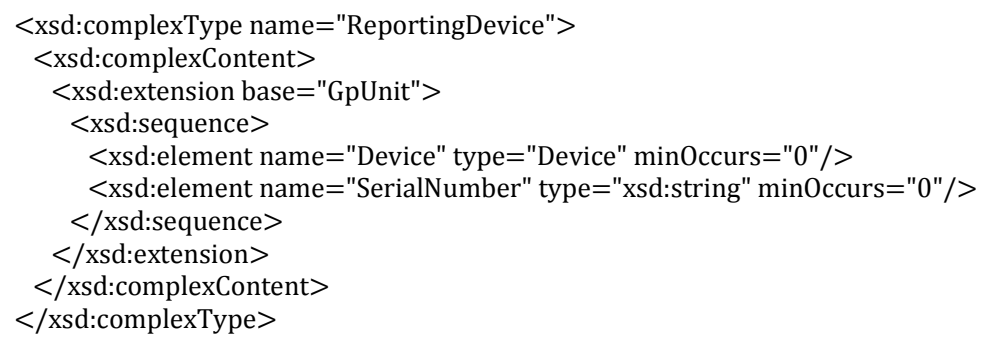




\subsubsection{The xsi:type=ReportingUnit}

For defining a geopolitical unit such as state, county, township, precinct, etc., using the $<$ ReportingUnitType > enumeration. It inherits the attributes and elements of <GpUnit> (see Table 4.29 and Table 4.30). Its syntax is:

$$
<\text { GpUnit xsi:type="ReportingUnit" } . . />
$$

This element optionally references < Person > to associate one or more individuals, e.g., authorities, for the reporting unit. It also includes < ContactInformation $>$ to provide contact addresses for the reporting unit, such as an address of a vote center.

$<$ Election $>$ references this element so as to identify the geographical scope of the election. In this case, the <GpUnit> element defined for the scope of the election may include $<$ ElectionAdministration > so as to include election authority-related information.

The <Type > sub-element uses the <ReportingUnitType > enumeration to specify the type of geopolitical geography being defined. <ReportingUnitType > contains the most common types of geographies, e.g., state, county, precinct, and so forth ${ }^{4}$. If the reporting unit type is not listed in enumeration <ReportingUnitType $>$, use other and include the reporting unit type (that is not listed in the enumeration) in <OtherType $>$.

The <IsDistricted $>$ boolean can be used in a number of ways. It is not strictly necessary, as it is possible to identify districts by their <Type $>$ sub-element or by examining the <Contest $>$ element's <ElectoralDistrictId > sub-element, which links to the electoral district associated with the contest (see section 4.2.6). However, if a district is defined but is not linked from a contest, or if the type of district is not listed in the <ReportingUnitType > enumeration and therefore $<$ OtherType $>$ is used, then <IsDistricted $>$ is necessary to identify the <GpUnit $>$ as a district. The $<$ IsDistricted $>$ boolean can also be used to signify that a <GpUnit $>$ defined as a jurisdiction, e.g., a county, is also used as a district for, e.g., county-wide contests.

Table 4.32 - Elements for <GpUnit xsi:type="ReportingUnit">

\begin{tabular}{|c|c|c|l|}
\hline Element & Multiplicity & \multicolumn{1}{|c|}{ Type } & \multicolumn{1}{|c|}{ Element Description } \\
\hline <AuthorityIds $>$ & 0 or 1 & xsd:IDREFS & $\begin{array}{l}\text { The } \text { objectId of one or more }<\text { Person }> \\
\text { elements describing an authority } \\
\text { responsible for the reporting unit. }\end{array}$ \\
\hline$<$ ContactInformation $>$ & 0 or 1 & ContactInformation & $\begin{array}{l}\text { For associating contact information } \\
\text { with the reporting unit. }\end{array}$ \\
\hline$<$ CountStatus $>$ & 0 or more & CountStatus & $\begin{array}{l}\text { For providing various counting status } \\
\text { on types of ballots or other items. }\end{array}$ \\
\hline
\end{tabular}

${ }^{4}$ A future version of this schema may specify geographies differently using a structured registry of defined types. 


\begin{tabular}{|c|c|c|c|}
\hline Element & Multiplicity & Type & Element Description \\
\hline$<$ ElectionAdministration $>$ & 0 or 1 & ElectionAdministration & $\begin{array}{l}\text { For use when the reporting unit serves } \\
\text { as the authority in the election. }\end{array}$ \\
\hline$<$ IsDistricted $>$ & 0 or 1 & xsd:boolean & $\begin{array}{l}\text { Boolean to indicate whether the } \\
\text { reporting unit is a district; assumed to } \\
\text { be "no" if not present. }\end{array}$ \\
\hline$<$ IsMailOnly $>$ & 0 or 1 & xsd:boolean & $\begin{array}{l}\text { Boolean to indicate whether the } \\
\text { reporting unit handles only mail-in or } \\
\text { absentee ballot elections, assumed to } \\
\text { be "no" if not present. }\end{array}$ \\
\hline$<$ Number $>$ & 0 or 1 & xsd:string & $\begin{array}{l}\text { A number associated with the } \\
\text { reporting unit; for compatibility with } \\
\text { VIP. }\end{array}$ \\
\hline$<$ PartyRegistration $>$ & 0 or more & PartyRegistration & $\begin{array}{l}\text { For associating a count of registered } \\
\text { voters per party with the geopolitical } \\
\text { unit. }\end{array}$ \\
\hline$<$ SpatialDimension $>$ & 0 or 1 & SpatialDimension & $\begin{array}{l}\text { For describing the reporting unit's } \\
\text { spatial extent (a polygon that shows } \\
\text { the related area). }\end{array}$ \\
\hline$<$ SubUnitsReported $>$ & 0 or 1 & xsd:integer & $\begin{array}{l}\text { Number of associated subunits such as } \\
\text { precincts that have completed } \\
\text { reporting. }\end{array}$ \\
\hline$<$ TotalSubUnits $>$ & 0 or 1 & xsd:integer & $\begin{array}{l}\text { Total number of associated subunits } \\
\text { such as precincts. }\end{array}$ \\
\hline$<$ Type $>$ & 1 & ReportingUnitType & $\begin{array}{l}\text { Enumerated type of reporting unit, } \\
\text { e.g., state, county, district, precinct, } \\
\text { etc. }\end{array}$ \\
\hline$<$ OtherType $>$ & 0 or 1 & xsd:string & $\begin{array}{l}\text { For use when <ReportingUnitType }> \\
\text { value is other. }\end{array}$ \\
\hline$<$ VotersParticipated $>$ & 0 or 1 & xsd:integer & $\begin{array}{l}\text { Number of voters who have } \\
\text { participated in the election, i.e., shown } \\
\text { up at the polls, including those who } \\
\text { did not cast ballots. }\end{array}$ \\
\hline$<$ VotersRegistered $>$ & 0 or 1 & xsd:integer & $\begin{array}{l}\text { Number of registered voters residing } \\
\text { within the boundaries of the } \\
\text { geopolitical unit. }\end{array}$ \\
\hline
\end{tabular}

\section{Schema Definition:}

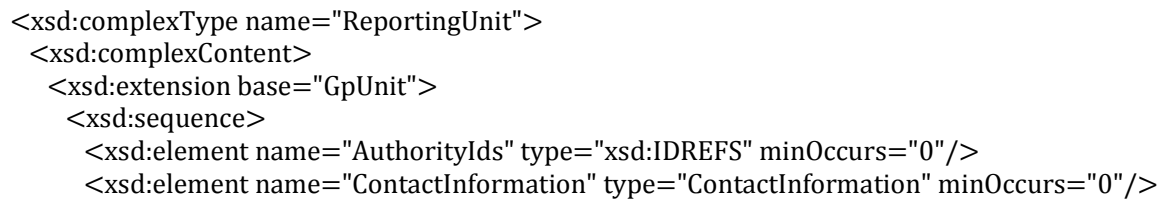


$<$ xsd:element name $=$ "CountStatus" type $=$ "CountStatus" minOccurs $=" 0 "$ maxOccurs $="$ unbounded" $/>$ $<$ xsd:element name="ElectionAdministration" type="ElectionAdministration" minOccurs="0" $>>$

$<$ xsd:element name="IsDistricted" type="xsd:boolean" minOccurs $=" 0 " />$

$<$ xsd:element name $="$ IsMailOnly" type $=$ "xsd:boolean" minOccurs $=" 0 " />$

$<$ xsd:element name="Number" type="xsd:string" minOccurs $=" 0 " />$

$<$ xsd:element name="PartyRegistration" type $=$ "PartyRegistration" minOccurs $=" 0 "$ maxOccurs $="$ unbounded" $/>$

$<$ xsd:element name $=$ "SpatialDimension" type $=$ "SpatialDimension" minOccurs="0" $/>$

$<x s d:$ element name $="$ SubUnitsReported" type $=$ "xsd:integer" minOccurs $=" 0 " />$

$<$ xsd:element name $=$ "TotalSubUnits" type $=$ "xsd:integer" minOccurs $=" 0 " />$

$<$ xsd:element name $="$ Type" type $=$ "ReportingUnitType" $/>$

$<$ xsd:element name $=$ "OtherType" type $=$ "xsd:string" minOccurs $=" 0 " />$

$<$ xsd:element name $=$ "VotersParticipated" type $=$ "xsd:integer" minOccurs $=" 0 " />$

$<$ xsd:element name $=$ "VotersRegistered" type $=$ "xsd:integer" minOccurs $=" 0 " />$

$</$ xsd:sequence $>$

$</$ xsd:extension $>$

$</$ xsd:complexContent $>$

$</$ xsd:complexType $>$ 


\subsubsection{The <InternationalizedText $>$ Element and <LanguageString > Complex Type}

For strings that can contain multi-national text, for use with text as shown on a ballot containing multi-national text. The Identifier attribute can be used to assign an identifier to the text as desired.

$<$ Text $>$ uses the xsd:language type such that its language attribute must be set to a value that identifies the language. An example of usage for the string “This is content in Spanish.” is as follows:

$<$ InternationalizedText $>$

$<$ Text Language $="$ es" $>$ Este es el contenido en Español. $</$ LanguageString $>$

$</$ InternationalizedText $>$

Values for language are from ISO 639 [12] and include:

- en - English

- en-US-U.S. English

- $\quad$ en-GB-U.K. English

- $f r-$ French

- es-Spanish

- $z h-$ Chinese

- ja-Japanese

- $k o-K o r e a n$

Table 4.33 - Attributes for <InternationalizedText>

\begin{tabular}{|c|c|c|c|}
\hline Attribute & Required & Type & Attribute Description \\
\hline label & no & xsd:string & For use as needed and compatibility with the VIP schema. \\
\hline
\end{tabular}

Table 4.34 - Elements for <InternationalizedText>

\begin{tabular}{|c|c|c|c|}
\hline Element & Multiplicity & Type & Element Description \\
\hline$<$ Text> & 1 or more & LanguageString & A string of text, i.e., possibly non-English. \\
\hline
\end{tabular}

Schema Definition:

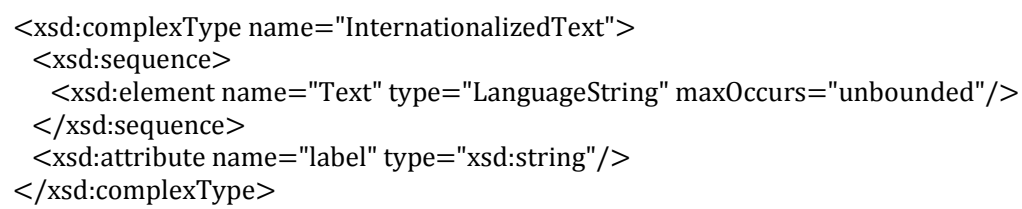


Table 4.35 - Attributes for $<$ Text $>$

\begin{tabular}{|c|c|c|c|}
\hline Attribute & Required & Type & Attribute Description \\
\hline language & yes & xsd:language & Identification of the language, e.g., es. \\
\hline
\end{tabular}

\section{Schema Definition:}

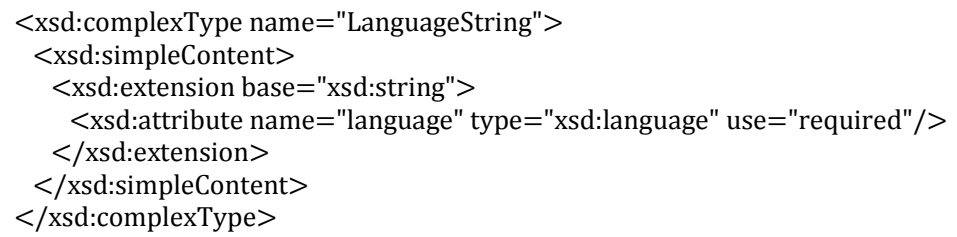




\subsubsection{The <Office> Element}

For defining an office and associated information associated with a contest and/or a district. $<$ ElectionReport $>$ includes $<$ Office $>$. <CandidateContest $>$ and $<$ RetentionContest $>$ reference $<$ Office $>$.

$<$ Office $>$ includes $<$ Term $>$ for defining details about the term of an office such as start/end dates and the type of term. <officeGroup > is included from ElectionReport (see section 4.2.12) to assign a name to a grouping of office definitions.

$<$ Office $>$ includes an optional <ElectoralDistrictId $>$ reference to a <GpUnit $>$ for the purpose of identifying the geographical scope of the office. For example, for an office for a state senate seat, <ElectoralDistrictId $>$ would include a reference to the <GpUnit $>$ defined for the district associated with that office.

Table 4.36 - Attributes for <Office>

\begin{tabular}{|c|c|c|l|}
\hline Attribute & Required & Type & \multicolumn{1}{|c|}{ Attribute Description } \\
\hline objectId & yes & xsd:ID & $\begin{array}{l}\text { Unique internal identifier used by other element(s) to } \\
\text { reference this element. }\end{array}$ \\
\hline
\end{tabular}

Table 4.37 - Elements for <Office>

\begin{tabular}{|c|c|c|c|}
\hline Element & Multiplicity & Type & Element Description \\
\hline$<$ ContactInformation $>$ & 0 or 1 & ContactInformation & $\begin{array}{l}\text { For associating various contact } \\
\text { information with the office. }\end{array}$ \\
\hline$<$ ElectoralDistrictId $>$ & 0 or 1 & xsd:IDREF & $\begin{array}{l}\text { Unique identifier for a <GpUnit }> \\
\text { element. For associating the office } \\
\text { with a reporting unit that represents } \\
\text { the geographical scope of the contest, } \\
\text { e.g., a district, etc. }\end{array}$ \\
\hline$<$ ExternalIdentifiers $>$ & 0 or 1 & ExternalIdentifiers & For associating an ID with the office. \\
\hline$<$ FilingDeadline $>$ & 0 or 1 & xsd:date & $\begin{array}{l}\text { Date and time when a candidate must } \\
\text { have filed for the contest for the } \\
\text { office. }\end{array}$ \\
\hline$<$ IsPartisan> & 0 or 1 & xsd:boolean & $\begin{array}{l}\text { Boolean to indicate whether the office } \\
\text { is partisan, e.g., true or false. If not } \\
\text { present, assumption is true. }\end{array}$ \\
\hline$<$ Name $>$ & 1 & InternationalizedText & $\begin{array}{l}\text { Name of the office; can appear on the } \\
\text { ballot. }\end{array}$ \\
\hline$<$ OfficeHolderPersonIds $>$ & 0 or 1 & xsd:IDREFS & $\begin{array}{l}\text { Unique identifier for one or more } \\
<\text { Candidate }>\text { elements defined for the } \\
\text { office holder. }\end{array}$ \\
\hline
\end{tabular}




\begin{tabular}{|c|c|c|l|}
\hline Element & Multiplicity & Type & \multicolumn{1}{|c|}{ Element Description } \\
\hline$<$ Term $>$ & 0 or 1 & & $\begin{array}{l}\text { For including office term-related } \\
\text { information. }\end{array}$ \\
\hline
\end{tabular}

Table 4.38 - Attributes for <Term>

\begin{tabular}{|c|c|c|c|}
\hline Attribute & Required & Type & Attribute Description \\
\hline label & no & xsd:string & For use as needed and compatibility with the VIP schema. \\
\hline
\end{tabular}

Table 4.39 - Elements for <Term $>$

\begin{tabular}{|c|c|c|l|}
\hline Element & Multiplicity & Type & \multicolumn{1}{|c|}{ Element Description } \\
\hline <StartDate $>$ & 0 or 1 & xsd:date & Start date for the current term of the office. \\
\hline <EndDate $>$ & 0 or 1 & xsd:date & End date for the current term of the office. \\
\hline <Type $>$ & 0 or 1 & OfficeTermType & $\begin{array}{l}\text { Enumerated type of term, e.g., full-term, } \\
\text { unexpired-term, etc. }\end{array}$ \\
\hline
\end{tabular}

\section{Schema Definition:}

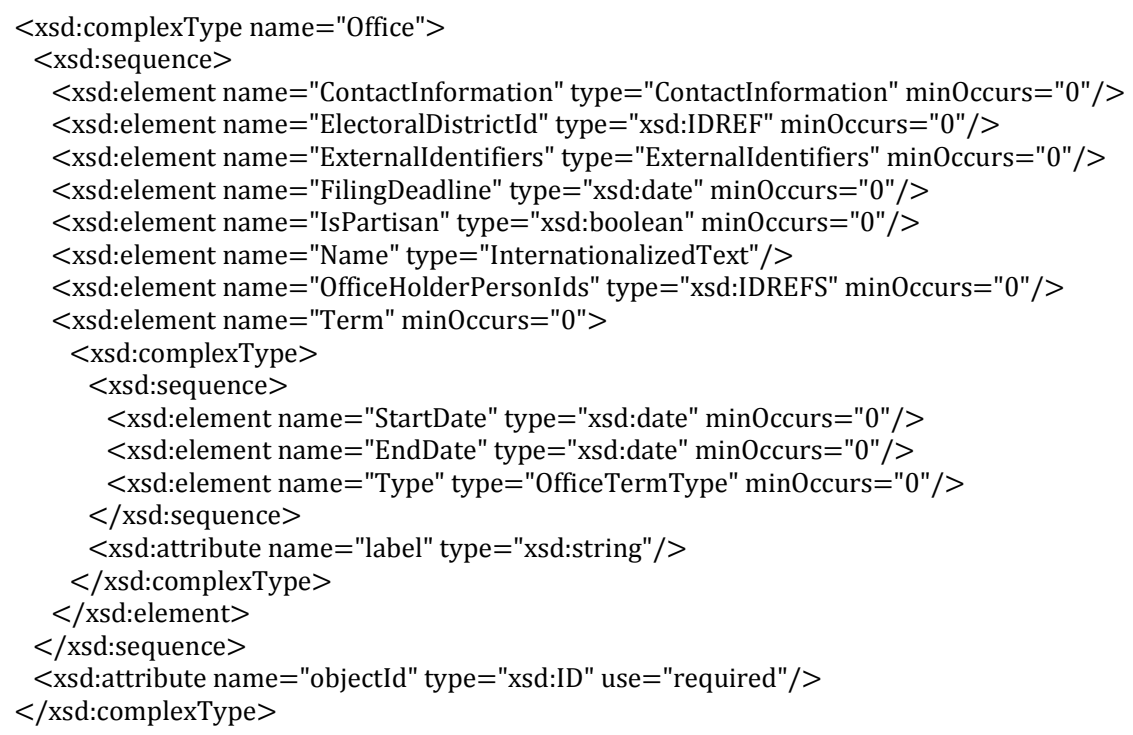




\subsubsection{The <OfficeGroup> Element}

Used to assign a name to a grouping of office definitions. It includes references to $<$ Office $>$ elements and a name to identify the grouping of references, e.g., "Judicial" or "Statewide", etc. $<$ SubOfficeGroup > can be used to create a nested hierarchy of groupings. <ElectionReport> includes $<$ OfficeGroup $>$.

As an example, <OfficeGroup> can be used to group state offices, etc., as follows:

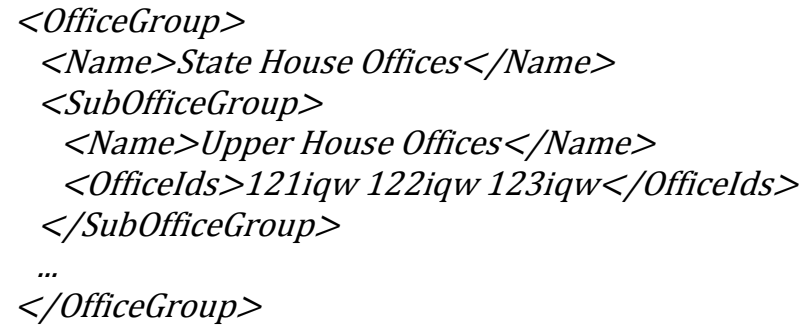

Table 4.40 - Attributes for <OfficeGroup>

\begin{tabular}{|c|c|c|c|}
\hline Attribute & Required & Type & Attribute Description \\
\hline label & no & xsd:string & For use as needed and compatibility with the VIP schema. \\
\hline
\end{tabular}

Table 4.41 - Elements for <OfficeGroup>

\begin{tabular}{|c|c|c|l|}
\hline Element & Multiplicity & Type & \multicolumn{1}{|c|}{ Element Description } \\
\hline$<$ Name $>$ & 1 & xsd:string & Name of the office grouping. \\
\hline$<$ OfficeIds $>$ & 0 or 1 & xsd:IDREFS & Unique identifier for one or more $<$ Office $>$ elements. \\
\hline$<$ SubOfficeGroup $>$ & 0 or more & OfficeGroup & $\begin{array}{l}\text { For defining a nested hierarchy of }<\text { Office }>\text { element } \\
\text { groupings. }\end{array}$ \\
\hline
\end{tabular}

Schema Definition:

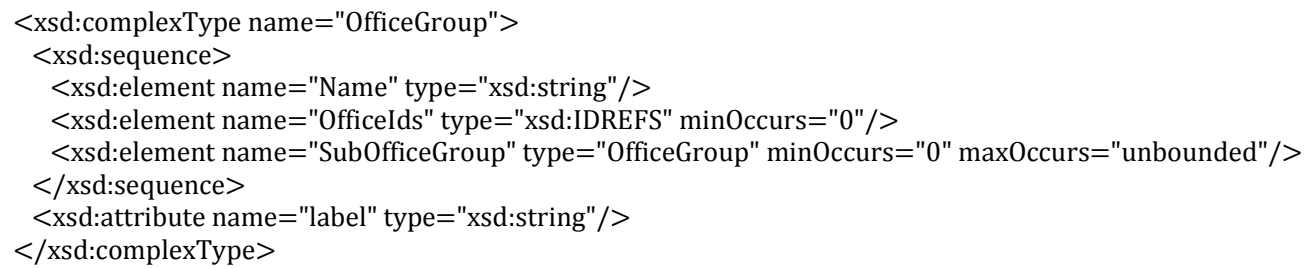




\subsubsection{The <Party> Element and Extension Base}

Used to describe a political party that can then be referenced in other elements. <ElectionReport $>$ includes $<$ Party $\rangle$. $<$ Candidate $>$, $<$ PartyContest $\rangle$, $<$ PartyRegistration $>$, and $<$ Person $>$ reference $<$ Party $>$.

$<$ Party $>$ is an abstract element with one xsi:type:

$<$ Party xsi:type="Coalition">, used to define coalitions (see section 4.2.18.1)

The <Color $>$ element specifies a 6-digit RGB code displayable using HTML. For example, the color green is encoded as:

$<$ Party Color="OOFFOO"... />

Table 4.42 - Attributes for <Party $>$

\begin{tabular}{|c|c|c|l|}
\hline Attribute & Required & Type & \multicolumn{1}{|c|}{ Attribute Description } \\
\hline objectId & yes & xsd:ID & $\begin{array}{l}\text { Unique internal identifier used by other element(s) to reference } \\
\text { this element. }\end{array}$ \\
\hline
\end{tabular}

Table 4.43 - Elements for <Party $>$

\begin{tabular}{|c|c|c|l|}
\hline Element & Multiplicity & Type & \multicolumn{1}{|c|}{ Element Description } \\
\hline <Abbreviation> & 0 or 1 & xsd:string & Short name for the party, e.g., "DEM”. \\
\hline$<$ Color $>$ & 0 or 1 & HTMLColorString & $\begin{array}{l}\text { For associating an HTML RGB color } \\
\text { coding with the party. }\end{array}$ \\
\hline$<$ ExternalIdentifiers $>$ & 0 or 1 & ExternalIdentifiers & For associating an ID with the party. \\
\hline$<$ LogoUri $>$ & 0 or 1 & xsd:anyUri & A URI to the party's graphical logo. \\
\hline$<$ Name $>$ & 1 & InternationalizedText & $\begin{array}{l}\text { Official full name of the party, e.g., } \\
\text { "Republican”; can appear on the ballot. }\end{array}$ \\
\hline
\end{tabular}

\section{Schema Definition:}

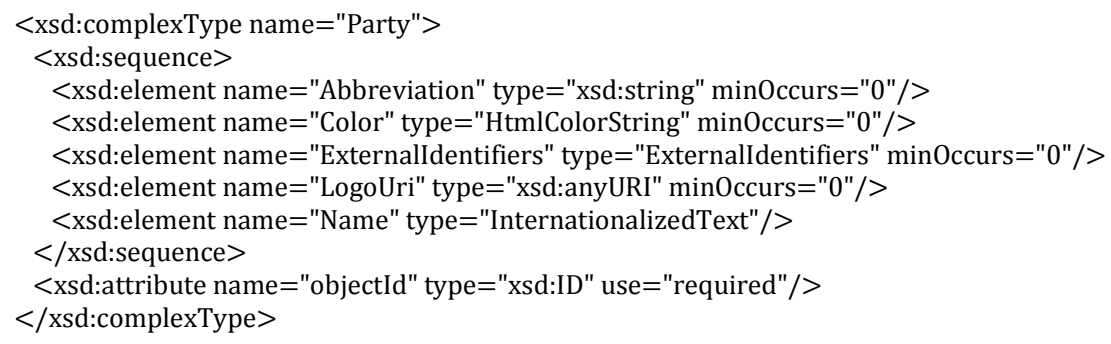




\subsubsection{The xsi:type=Coalition}

For defining a coalition, i.e., a collection of parties organized for the purpose of endorsing a candidates in a contest. It inherits the attributes and elements of <Party $>$ (see Table 4.42 and Table 4.43). Its syntax is:

$$
<\text { Party xsi:type="Coalition" } \ldots />
$$

$<$ Coalition $>$ elements are created by defining $<$ Party $>$ elements from $<$ ElectionReport $>$, but using the above syntax (thus, coalition elements can be referenced via any references to $<$ Party $>$ included in other elements, e.g., <Candidate $>$ or $<$ CandidateSelection $>$ ). $<$ Coalition $>$ elements themselves are composed of multiple <Party $>$ references along with a reference to an associated contest(s), for example:

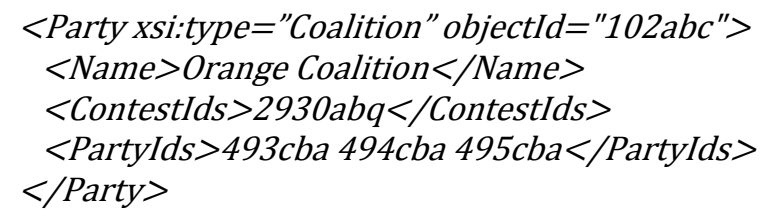

If $<$ ContestIds $>$ is not present, a general default is that the coalition endorses the associated parties.

Table 4.44 - Elements for <Party xsi:type="Coalition">

\begin{tabular}{|c|c|c|l|}
\hline Element & Multiplicity & Type & \multicolumn{1}{|c|}{ Element Description } \\
\hline$<$ ContestIds $>$ & 0 or 1 & xsd:IDREFS & $\begin{array}{l}\text { Unique identifier for one or more < Contest> elements. For } \\
\text { associating contests with the coalition. }\end{array}$ \\
\hline$<$ PartyIds $>$ & 0 or 1 & xsd:IDREFS & $\begin{array}{l}\text { Unique identifier for one or more }<\text { Party }>\text { elements. For } \\
\text { associating parties with the coalition. }\end{array}$ \\
\hline
\end{tabular}

Schema Definition:

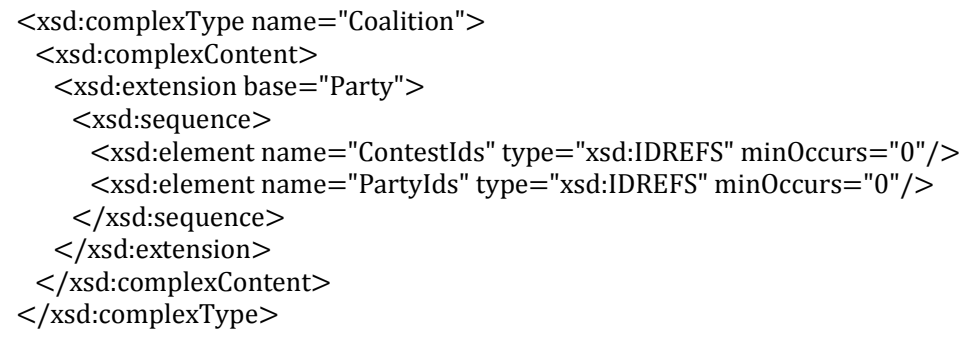




\subsubsection{The <PartyRegistration> Element}

For tracking the number of registered voters per party per geopolitical unit, i.e., for reporting on the number of registered voters of a particular party in a district or other type of reporting unit. Referenced by <GpUnit>.

Table 4.45 - Elements for <PartyRegistration>

\begin{tabular}{|c|c|c|l|}
\hline Element & Multiplicity & Type & \multicolumn{1}{|c|}{ Element Description } \\
\hline$<$ Count $>$ & 1 & xsd:integer & A count for tracking the number of registered voters. \\
\hline$<$ PartyId $>$ & 1 & xsd:IDREF & $\begin{array}{l}\text { Unique identifier for a }<\text { Party }>\text { element. For associating a } \\
\text { political party. }\end{array}$ \\
\hline
\end{tabular}

Schema Definition:

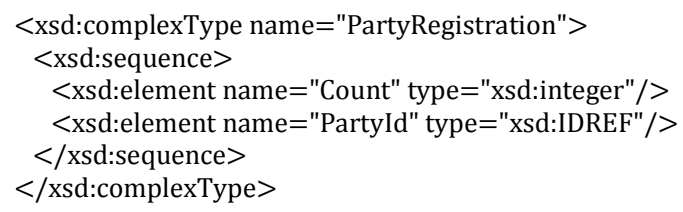




\subsubsection{The <Person> Element}

For defining information about a person; the person may be a candidate, election official, authority for a reporting unit, etc. <ElectionReport $>$ includes $\langle$ Person $\rangle$. $<$ Candidate $\rangle$ and $<$ ElectionAuthority $>$ and $<$ GpUnit $>$ reference $<$ Person $>$. $<$ Person $>$ optionally includes $<$ ContactInformation $>$ for associating contact information.

Multiple occurrences of the <MiddleName> element can be used as needed, e.g., for names such as “John Andrew Winston Smith”, as follows:

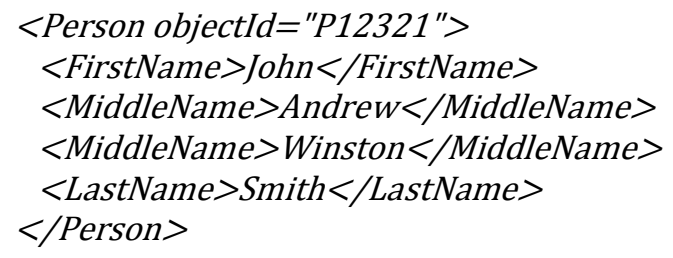

Table 4.46 - Attributes for <Person>

\begin{tabular}{|c|c|c|l|}
\hline Attribute & Required & Type & \multicolumn{1}{|c|}{ Attribute Description } \\
\hline objectld & yes & xsd:ID & $\begin{array}{l}\text { Unique internal identifier used by other element(s) to reference } \\
\text { this element. }\end{array}$ \\
\hline
\end{tabular}

Table 4.47 - Elements for <Person>

\begin{tabular}{|c|c|c|l|}
\hline Element & Multiplicity & \multicolumn{1}{|c|}{ Type } & \multicolumn{1}{|c|}{ Element Description } \\
\hline$<$ ContactInformation $>$ & 0 or more & ContactInformation & $\begin{array}{l}\text { For associating contact information with } \\
\text { the person. }\end{array}$ \\
\hline$<$ DateOfBirth $>$ & 0 or 1 & xsd:date & Person's date of birth. \\
\hline$<$ FirstName $>$ & 0 or 1 & xsd:string & Person's first (given) name. \\
\hline$<$ FullName $>$ & 0 or 1 & InternationalizedText & Person's full name. \\
\hline$<$ Gender $>$ & 0 or 1 & xsd:string & Person's gender. \\
\hline$<$ LastName $>$ & 0 or 1 & xsd:string & Person's last (family) name. \\
\hline$<$ MiddleName $>$ & 0 or more & xsd:string & Person's middle name. \\
\hline$<$ Nickname $>$ & 0 or 1 & xsd:string & Nickname associated with the person. \\
\hline$<$ PartyId $>$ & 0 or 1 & xsd:IDREF & $\begin{array}{l}\text { Unique identifier for a Party element. } \\
\text { For associating a political party with the } \\
\text { person. }\end{array}$ \\
\hline$<$ Prefix $>$ & 0 or 1 & xsd:string & $\begin{array}{l}\text { A prefix associated with the person, e.g., } \\
\text { Mr. }\end{array}$ \\
\hline$<$ Profession $>$ & 0 or 1 & InternationalizedText & Person's profession. \\
\hline$<$ Suffix $>$ & 0 or 1 & xsd:string & $\begin{array}{l}\text { A suffix associated with the person, e.g., } \\
\text { Jr. }\end{array}$ \\
\hline
\end{tabular}




\begin{tabular}{|c|c|c|c|}
\hline Element & Multiplicity & Type & Element Description \\
\hline$<$ Title $>$ & 0 or 1 & InternationalizedText & A title associated with the person. \\
\hline
\end{tabular}

\section{Schema Definition:}

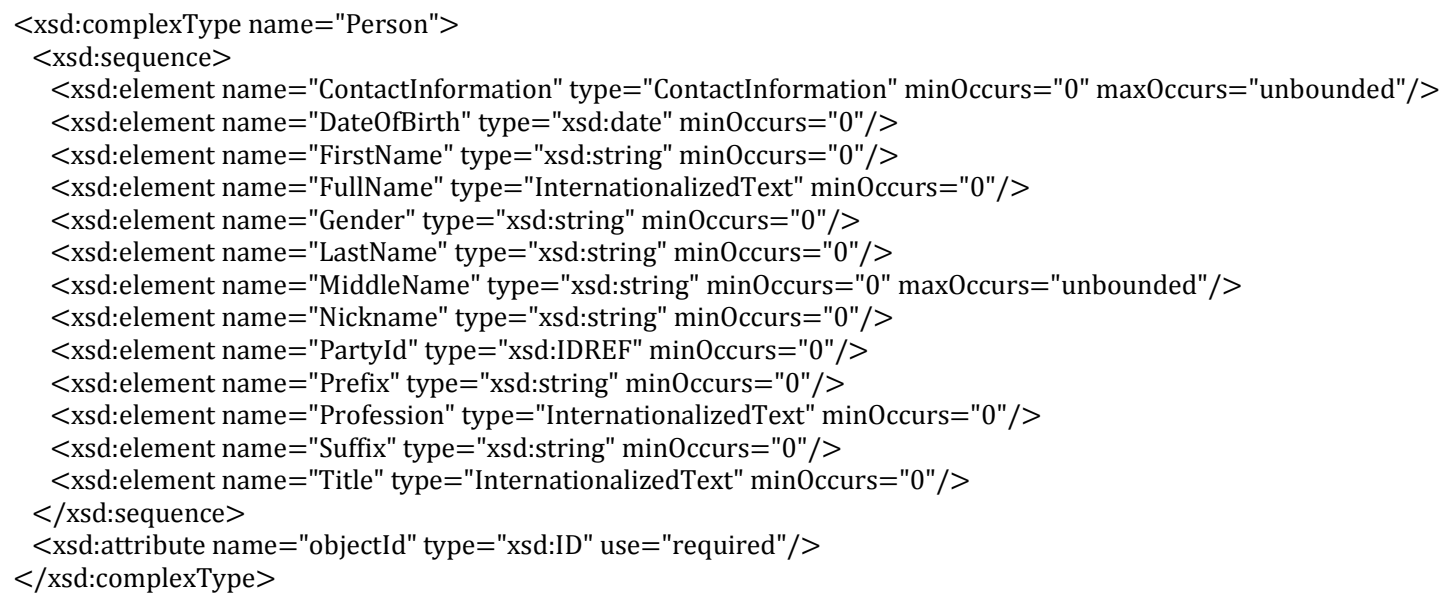




\subsubsection{The <Schedule> and <Hours $>$ Elements}

For defining a schedule associated with a particular election office or location. $<$ ContactInformation $>$ includes $<$ Schedule $>$.

Table 4.48 - Attributes for <Schedule>

\begin{tabular}{|c|c|c|l|}
\hline Attribute & Required & Type & \multicolumn{1}{|c|}{ Attribute Description } \\
\hline label & no & xsd:string & $\begin{array}{l}\text { For use as needed and compatibility with the VIP } \\
\text { schema. }\end{array}$ \\
\hline
\end{tabular}

Table 4.49 - Elements for <Schedule>

\begin{tabular}{|c|c|c|l|}
\hline Element & Multiplicity & Type & \multicolumn{1}{|c|}{ Element Description } \\
\hline$<$ Hours $>$ & 0 or more & Hours & For specifying a range of hours for a schedule. \\
\hline$<$ IsOnlyByAppointmment> & 0 or 1 & xsd:boolean & $\begin{array}{l}\text { If an appointment is only by appointment; } \\
\text { assumed to be “no" if not present. }\end{array}$ \\
\hline <IsOrByAppointment $>$ & 0 or 1 & xsd:boolean & $\begin{array}{l}\text { If an appointment can by appointment } \\
\text { presumably as desired; assumed to be "no" if not } \\
\text { present. }\end{array}$ \\
\hline$<$ IsSubjectToChange $>$ & 0 or 1 & xsd:boolean & $\begin{array}{l}\text { If an appointment may be subject to change; } \\
\text { assumed to be "no" if not present. }\end{array}$ \\
\hline$<$ StartDate> & 0 or 1 & xsd:date & For the starting date of the schedule. \\
\hline <EndDate> & 0 or 1 & xsd:date & For the ending date of the schedule. \\
\hline
\end{tabular}

\section{Schema Definition:}

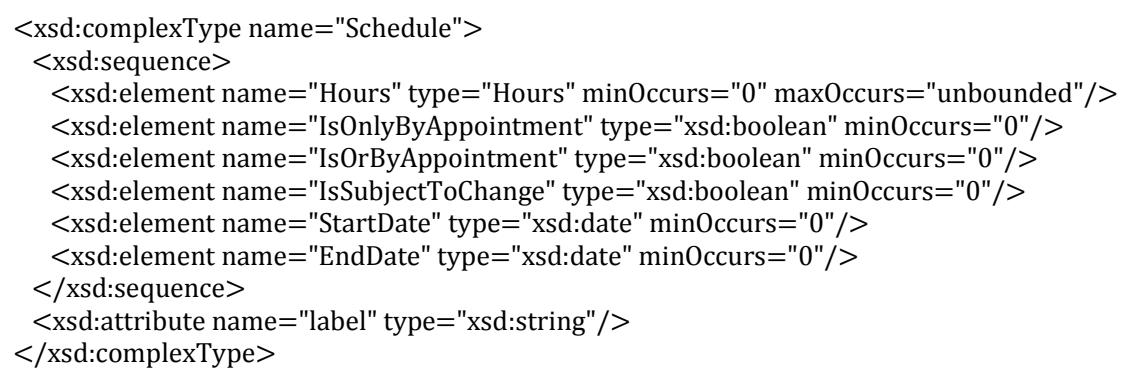




\subsubsection{The <Hours> Element}

$<$ Hours $>$ is used to specify a specific day and hours on that day, including the time zone. Multiple occurences of <Hours > can be used if the schedule includes a range of days and hours, for example, for specific hours on a Wednesday and Thursday:

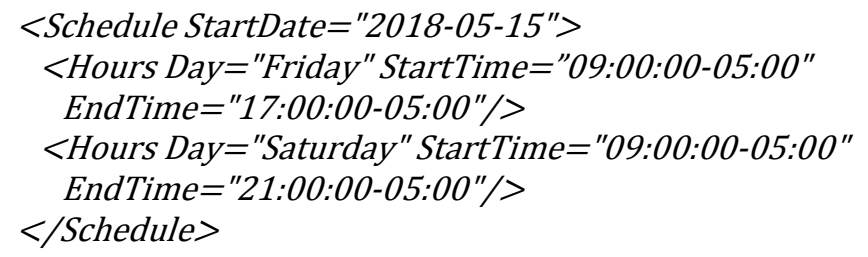

Table 4.50 - Attributes for <Hours $>$

\begin{tabular}{|c|c|c|l|}
\hline Attribute & Required & Type & \multicolumn{1}{|c|}{ Attribute Description } \\
\hline label & no & xsd:string & $\begin{array}{l}\text { For use as needed and compatibility with the VIP } \\
\text { schema. }\end{array}$ \\
\hline
\end{tabular}

Table 4.51 - Elements for <Hours>

\begin{tabular}{|c|c|c|l|}
\hline Element & Multiplicity & Type & \multicolumn{1}{c|}{ Element Description } \\
\hline$<$ Day $>$ & 0 or 1 & DayType & Day of week or weekend. \\
\hline$<$ StartTime $>$ & 1 & TimeWithZone & Start time of the schedule. \\
\hline$<$ EndTime $>$ & 1 & TimeWithZone & End time of the schedule. \\
\hline
\end{tabular}

\section{Schema Definition:}

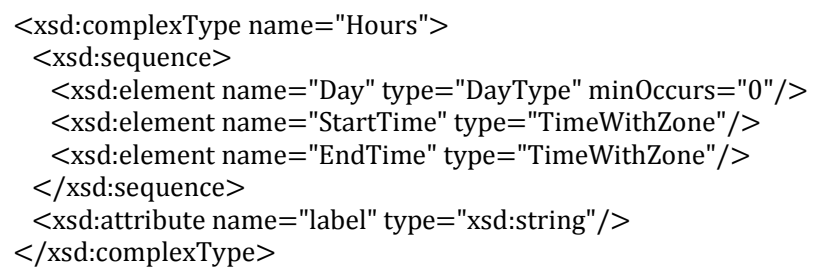




\subsubsection{The <SpatialDimension> and <SpatialExtent $>$ Elements}

For defining the spatial layout of a <GpUnit>, e.g., a map or a spatial extent (a polygon that shows the related area) for various purposes, including to visualize election results, to understand the composition of districts, or to determine whether <GpUnit> elements are properly related. $<$ GpUnit $>$ includes < SpatialDimension $>$.

Table 4.52 - Elements for <SpatialDimension>

\begin{tabular}{|c|c|c|l|}
\hline Element & Multiplicity & Type & \multicolumn{1}{|c|}{ Element Description } \\
\hline$<$ MapUri $>$ & 0 or 1 & xsd:anyURI & Typically a URL to a map of the <GpUnit $>$. \\
\hline < SpatialExtent> & 0 or 1 & SpatialExtent & $\begin{array}{l}\text { For associating a <GpUnit> element's spatial extent } \\
\text { information. }\end{array}$ \\
\hline
\end{tabular}

\section{Schema Definition:}

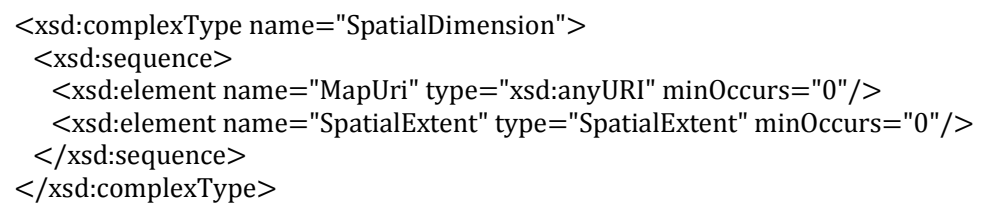




\subsubsection{The SpatialExtent Element}

$<$ SpatialDimension > includes < SpatialExtent $>$ for defining a <GpUnit > element's spatial extent data and the format used for the spatial extent.

Table 4.53 - Elements for <SpatialExtent>

\begin{tabular}{|c|c|c|l|}
\hline Element & Multiplicity & Type & \multicolumn{1}{|c|}{ Element Description } \\
\hline$<$ Coordinates $>$ & 1 & xsd:string & The data coordinates constituting the spatial extent. \\
\hline$<$ Format $>$ & 1 & GeoSpatialFormat & $\begin{array}{l}\text { Enumerated type for the format used, e.g., } \mathrm{gml}, \mathrm{kml}, \\
w \mathrm{kt} \text {, etc. }\end{array}$ \\
\hline
\end{tabular}

Schema Definition:

$<$ xsd:complexType name="SpatialExtent" >

$<$ xsd:sequence $>$

$<$ xsd:element name $=$ "Coordinates" type $=$ "xsd:string" $/>$

$<$ xsd:element name $=$ "Format" type $=$ "GeoSpatialFormat" $/>$

$</$ xsd:sequence $>$

$</$ xsd:complexType $>$ 


\subsubsection{The <SummaryCounts $>$ (extension base <Counts $>$ ) Element}

For reporting on contest-wide or geopolitical unit-wide summary counts. Includes $<$ Counts $>$ as an extension base and therefore inherits the elements from <Counts $>$ (see Table 4.22), but it is included directly from <Contest $>$ and $<$ GpUnit $>$ (i.e., not as an xsi:type of <Counts $>$ ).

$<$ Contest $>$ includes < SummaryCounts $>$ for providing a geographical scope-wide summary of miscellaneous counts associated with a contest, including total number of ballots cast containing the contest, total number of overvotes, undervotes, and write-ins. < SummaryCounts $>$ can optionally reference <GpUnit> elements defined for lower level reporting units, e.g., precincts, so as to associate summary counts with each precinct or other lower-level reporting units within the scope of the contest.

$<$ GpUnit $>$ includes <SummaryCounts $>$ for providing summary counts specific to that <GpUnit $>$, e.g., a $<$ GpUnit $>$ defined for a district can include $<$ SummaryCounts $>$ for this purpose.

$<$ SummaryCounts $>$, in this case, would not include the <GpUnitId $>$ element in $<$ Counts $>$.

In $<$ Counts $>$, if the type of count item is not listed in enumeration <CountItemType $>$, use other and include the type (that is not listed in the enumeration) in <OtherType $>$.

Table 4.54 - Elements for <SummaryCounts>

\begin{tabular}{|c|c|c|l|}
\hline Element & Multiplicity & Type & \multicolumn{1}{|c|}{ Element Description } \\
\hline <BallotsCast $>$ & 0 or 1 & xsd:integer & Number of ballots cast. \\
\hline <BallotsOutstanding $>$ & 0 or 1 & xsd:integer & Number of ballots not yet counted. \\
\hline$<$ BallotsRejected $>$ & 0 or 1 & xsd:integer & Number of ballots rejected. \\
\hline$<$ Overvotes $>$ & 0 or 1 & xsd:integer & Number of overvotes. \\
\hline$<$ Undervotes $>$ & 0 or 1 & xsd:integer & Number of undervotes. \\
\hline$<$ WriteIns $>$ & 0 or 1 & xsd:integer & Number of write-ins. \\
\hline
\end{tabular}

Schema Definition:

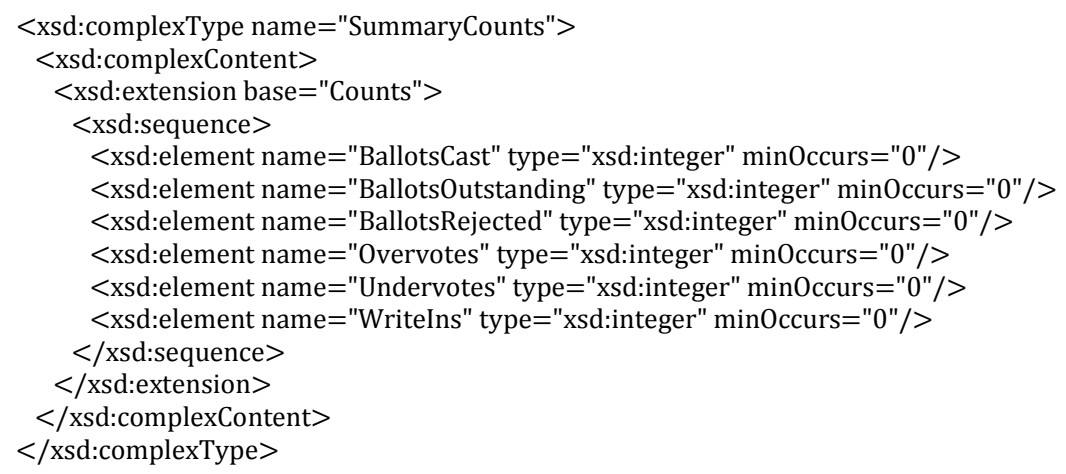




\subsubsection{The <VoteCounts $>$ (extension base <Counts $>$ ) Element}

For reporting on vote counts for ballot selections in a contest. <VoteCounts $>$ includes $<$ Counts $>$ as an extension base and therefore inherits the elements from <Counts $>$ (see Table 4.22), but it is included directly by <BallotSelection $>$.

\section{Table 4.55 - Elements for <VoteCounts>}

\begin{tabular}{|c|c|c|l|}
\hline Element & Multiplicity & Type & \multicolumn{1}{|c|}{ Element Description } \\
\hline$<$ Count $>$ & 0 or 1 & xsd:float & $\begin{array}{l}\text { Count of contest votes cast; can include a fractional component } \\
\text { in special cases. }\end{array}$ \\
\hline
\end{tabular}

\section{Schema Definition:}

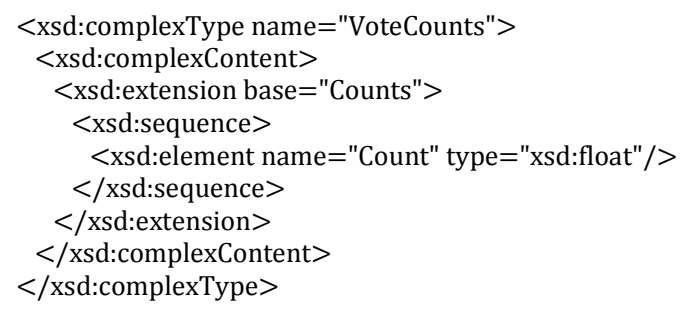




\subsection{Enumerations}

The following sections deal with simple type enumerations in the schema.

\subsubsection{The BallotMeasureType Enumeration}

Enumeration for types of ballot measures in the <BallotMeasureContest $>$ element.

Table 4.56 - Values for <BallotMeasureType>

\begin{tabular}{|c|l|}
\hline Value & \multicolumn{1}{|c|}{ Value Description } \\
\hline ballot-measure & For a standard “yes” or “no" question on the ballot. \\
\hline initiative & For an initiative. \\
\hline referendum & For a referendum. \\
\hline other & $\begin{array}{l}\text { Used when the type of ballot measure is not included in this } \\
\text { enumeration. }\end{array}$ \\
\hline
\end{tabular}

\section{Schema Definition:}

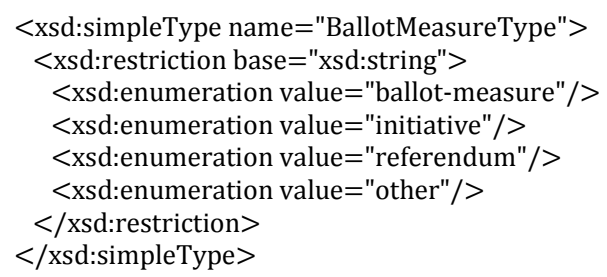




\subsubsection{The CandidatePostElectionStatus Enumeration}

Enumeration for various post-election statuses applicable to a candidate in the <Candidate> element.

Table 4.57 - Values for <CandidatePostElectionStatus>

\begin{tabular}{|c|l|}
\hline \multicolumn{1}{|c|}{ Value } & \multicolumn{1}{c|}{ Value Description } \\
\hline advanced-to-runoff & For candidates who have advanced to a runoff. \\
\hline projected-winner & For a projected contest winner. \\
\hline winner & $\begin{array}{l}\text { For the official contest winner or one of “n” contest winners for n-of-m } \\
\text { voting. }\end{array}$ \\
\hline withdrawn & For candidates who have withdrawn from the contest. \\
\hline
\end{tabular}

\section{Schema Definition:}

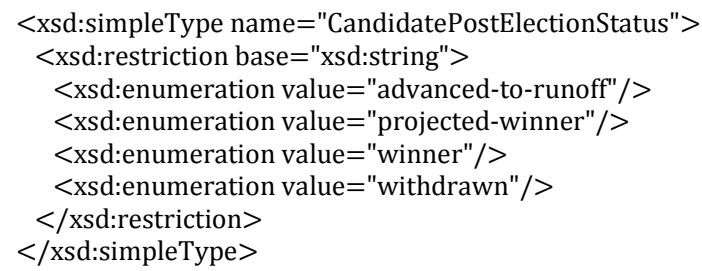




\subsubsection{The CandidatePreElectionStatus Enumeration}

Enumeration for various pre-election statuses applicable to a candidate in the $<$ Candidate $>$ element.

Table 4.58 - Values for <CandidatePreElectionStatus>

\begin{tabular}{|c|l|}
\hline Value & \multicolumn{1}{|c|}{ Value Description } \\
\hline filed & For candidates who have filed with the election authority but not necessarily qualified. \\
\hline qualified & $\begin{array}{l}\text { For candidates who are qualified by the election authority to be on the ballot for a } \\
\text { contest. }\end{array}$ \\
\hline withdrawn & For candidates who have withdrawn from the contest. \\
\hline write-in & For when the candidate is a write-in. \\
\hline
\end{tabular}

\section{Schema Definition:}

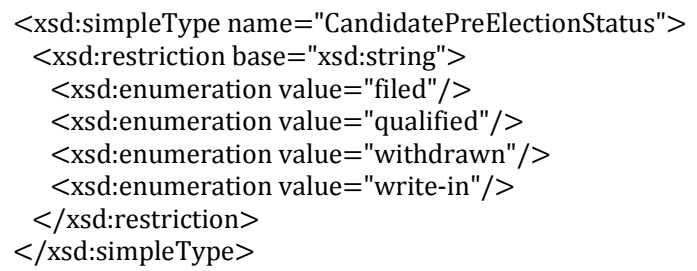




\subsubsection{The CountltemStatus Enumeration}

Enumeration for various counting-related statuses for types of ballots or write-ins, used in the $<$ CountStatus $>$ element.

Table 4.59 - Values for <CountltemStatus>

\begin{tabular}{|c|l|}
\hline Value & \multicolumn{2}{|c|}{ Value Description } \\
\hline completed & For counts that are complete. \\
\hline in-process & For counts that are in process \\
\hline not-processed & When the counting has not started or is not underway. \\
\hline unknown & When the status of the counting is unknown. \\
\hline
\end{tabular}

\section{Schema Definition:}

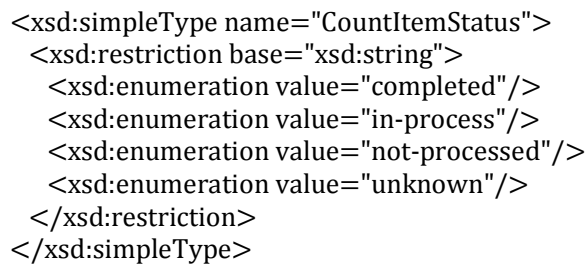




\subsubsection{The CountltemType Enumeration}

Enumeration for the items that are counted during the course of an election and for which the status of the counts are of interest. Used in the $<$ Counts $>$ and $<$ CountStatus $>$ elements.

Table 4.60 - Values for <CountltemType>

\begin{tabular}{|c|l|}
\hline Value & \multicolumn{1}{|c|}{ Value Description } \\
\hline absentee & $\begin{array}{l}\text { For any/all types of absentee, generally when absentee is not broken out into } \\
\text { specific types. }\end{array}$ \\
\hline absentee-fwab & A type of absentee; for Federal Write-in Absentee Ballots. \\
\hline absentee-in-person & $\begin{array}{l}\text { A type of absentee; for absentee ballots cast in-person, e.g., at a county } \\
\text { office. }\end{array}$ \\
\hline absentee-mail & A type of absentee; for postal mail absentee ballots. \\
\hline early & For ballots cast during early voting periods. \\
\hline election-day & For ballots cast on election day. \\
\hline provisional & For challenged ballots. \\
\hline total & For the total of all ballot types. \\
\hline uocava & $\begin{array}{l}\text { For ballots from UOCAVA (Uniform and Overseas Citizens Assistance in } \\
\text { Voting Act) voters. }\end{array}$ \\
\hline write-in & For write-ins on ballots. \\
\hline other & Used when the type of counting item is not included in this enumeration. \\
\hline
\end{tabular}

\section{Schema Definition:}

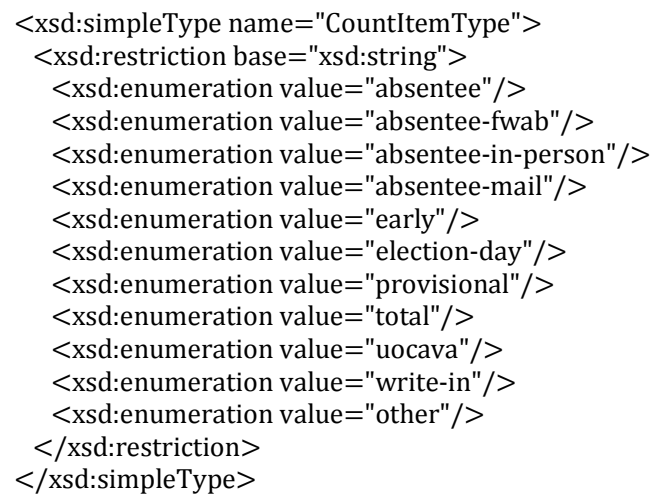




\subsubsection{The DayType Enumeration}

Enumeration for the day(s) in a schedule in the $<$ Schedule $>$ element.

Table 4.61 - Values for <DayType>

\begin{tabular}{|c|c|}
\hline Value & Value Description \\
\hline all & Used for all days of the week. \\
\hline sunday & \\
\hline monday & \\
\hline tuesday & \\
\hline wednesday & \\
\hline thursday & \\
\hline friday & \\
\hline saturday & \\
\hline weekday & Used for any day of the week. \\
\hline weekend & Used for both Saturday and Sunday. \\
\hline
\end{tabular}

\section{Schema Definition:}

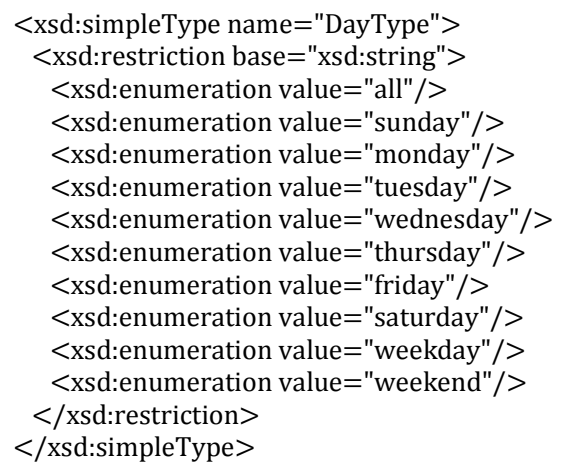




\subsubsection{The DeviceType Enumeration}

Enumeration for the type of device in the $<$ Device $>$ element.

Table 4.62 - Values for <DeviceType>

\begin{tabular}{|c|l|}
\hline Value & \multicolumn{1}{|c|}{ Value Description } \\
\hline electronic & For DRE (Direct Record Electronic) and touchscreen devices such as tablets. \\
\hline lever & For lever machines. \\
\hline manual-count & For hand-counted paper ballots. \\
\hline mixed-systems & $\begin{array}{l}\text { For devices, e.g., that print voter choices on an optical scan ballot (hybrid of a } \\
\text { DRE and an optical scan system) }\end{array}$ \\
\hline opscan-central & $\begin{array}{l}\text { For an optical scanner used at a central office with no opportunity for voter } \\
\text { correction of mistakes. }\end{array}$ \\
\hline opscan-precinct & $\begin{array}{l}\text { For an optical scanner used at a precinct or other location where voter correction } \\
\text { of mistakes such as overvotes is possible. }\end{array}$ \\
\hline punch-card & For punch card devices. \\
\hline unknown & Used when the type of device is unknown. \\
\hline other & Used when the device type is not listed in this enumeration. \\
\hline
\end{tabular}

\section{Schema Definition:}

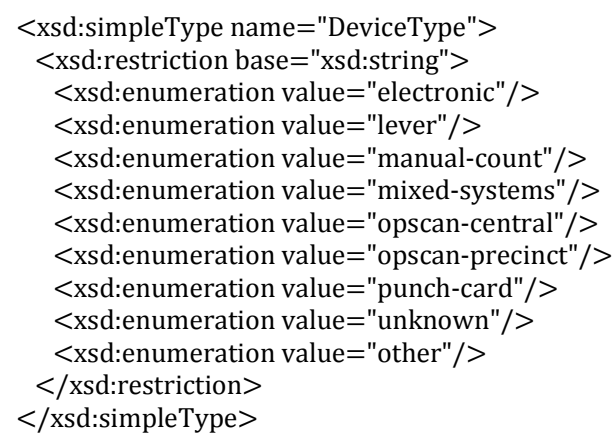




\subsubsection{The ElectionType Enumeration}

Enumeration for the type of election in the <ElectionReport> element.

Table 4.63 - Values for <ElectionType>

\begin{tabular}{|c|l|}
\hline Value & \multicolumn{1}{|c|}{ Value Description } \\
\hline general & $\begin{array}{l}\text { For the election held typically on the national day for elections (e.g., } \\
\text { the Tuesday after the } 1^{\text {st }} \text { Monday in November). }\end{array}$ \\
\hline partisan-primary-closed & $\begin{array}{l}\text { For a primary election that is for a specific party where voter eligibility } \\
\text { is based on registration. }\end{array}$ \\
\hline partisan-primary-open & $\begin{array}{l}\text { For a primary election that is for a specific party where voter declares } \\
\text { desired party or chooses in private. }\end{array}$ \\
\hline primary & For a primary election, type not specified such as nonpartisan. \\
\hline runoff & $\begin{array}{l}\text { For an election to decide a prior contest that ended with no candidate } \\
\text { receiving a majority of the votes. }\end{array}$ \\
\hline special & $\begin{array}{l}\text { For an election held out of sequence for special circumstances, e.g., to } \\
\text { fill a vacated office. }\end{array}$ \\
\hline other & Used when the election type is not listed in this enumeration. \\
\hline
\end{tabular}

Schema Definition:

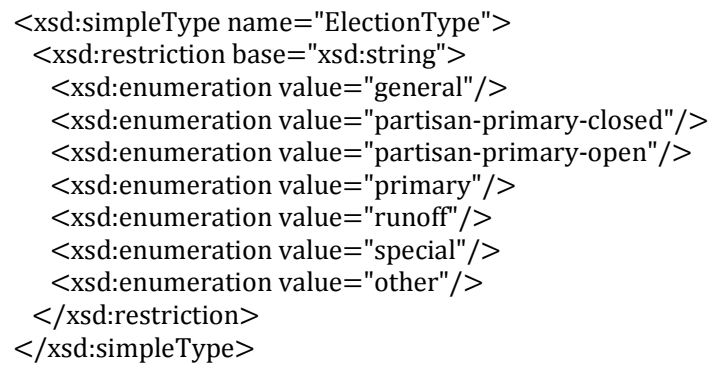




\subsubsection{The GeoSpatialFormat Enumeration}

Enumeration for geospatial vector data formats used in geographic information system (GIS) software, used in the <SpatialExtent> element.

Table 4.64 - Values for <GeoSpatialFormat>

\begin{tabular}{|c|l|}
\hline Value & \multicolumn{1}{|c|}{ Value Description } \\
\hline geo-json & For GeoJSON open standard format. \\
\hline gml & For Geography Markup Language format. \\
\hline $\mathrm{kml}$ & For Keyhole Markup Language format. \\
\hline $\mathrm{shp}$ & For the shape file format associated with Esri. \\
\hline wkt & For Well-known Text format. \\
\hline
\end{tabular}

\section{Schema Definition:}

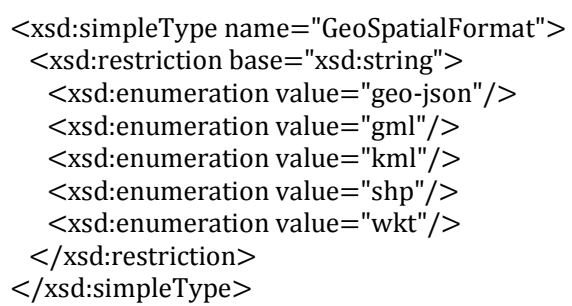




\subsubsection{The IdentifierType Enumeration}

Enumeration for election data-related codes in the $<$ ExternalIdentifiers $>$ element ${ }^{5}$.

Table 4.65 - Values for <IdentifierType>

\begin{tabular}{|c|l|}
\hline Value & \\
\hline fips & For FIPS codes. \\
\hline local-level & For a code that is specific to a county or other similar locality. \\
\hline national-level & For a code that is used at the national level other than "fips" or "ocd-id". \\
\hline ocd-id & For Open Civic Data identifiers. \\
\hline state-level & For a code that is specific to a state. \\
\hline other & Used when the type of code is not included in this enumeration. \\
\hline
\end{tabular}

\section{Schema Definition:}

$<$ xsd:simpleType name="IdentifierType" $>$

$<$ xsd:restriction base $=$ "xsd:string" $>$

$<$ xsd:enumeration value $=$ "fips" $/>$

$<$ xsd:enumeration value $=$ "local-level" $/>$

$<$ xsd:enumeration value $=$ "national-level" $/>$

$<$ xsd:enumeration value $=$ "ocd-id" $/>$

$<$ xsd:enumeration value $=$ "state-level" $/>$

$<$ xsd:enumeration value $=$ "other" $/>$

$</$ xsd:restriction $>$

$</$ xsd:simpleType $>$

\footnotetext{
${ }^{5}$ A future version of this schema may incorporate a registry of election data-related codes.
} 


\subsubsection{The OfficeTermType Enumeration}

Enumeration for the office term type in the <office $>$ element.

Table 4.66 - Values for <OfficeTermType>

\begin{tabular}{|c|l|}
\hline Value & \multicolumn{1}{|c|}{ Value Description } \\
\hline full-term & $\begin{array}{l}\text { When the officeholder's term began at the beginning of the full term of the office, } \\
\text { e.g., } 6 \text { years for U.S. Senate. }\end{array}$ \\
\hline unexpired-term & $\begin{array}{l}\text { When the officeholder's term began at some date after the beginning of the full } \\
\text { term of the office, generally because the previous officeholder vacated the office } \\
\text { before the fullterm expired. }\end{array}$ \\
\hline
\end{tabular}

Schema Definition:

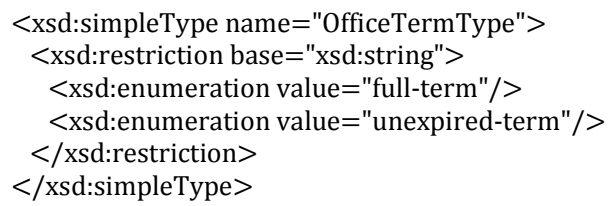




\subsubsection{The ReportDetailLevel Enumeration}

Enumeration for the detail level of the election results report in the <Election> element.

Table 4.67 - Values for <ReportDetailLevel>

\begin{tabular}{|c|l|}
\hline Value & \multicolumn{1}{|c|}{ Value Description } \\
\hline precinct-level & For reports that contain counts from precincts in the reporting jurisdiction. \\
\hline summary-contest & For reports that contain only aggregated counts. \\
\hline
\end{tabular}

Schema Definition:

$<$ xsd:simpleType name="ReportDetailLevel" $>$ $<x$ sd:restriction base $=$ "xsd:string" $>$

$<$ xsd:enumeration value $=$ "precinct-level" $/>$

$<$ xsd:enumeration value $=$ "summary-contest" $/>$

$</$ xsd:restriction $>$

$</$ xsd:simpleType $>$ 


\subsubsection{The ReportingUnitType Enumeration}

Enumeration for the type of geopolitical unit in the <GpUnit xsi:type="ReportingUnit" $>$ element ${ }^{6}$.

Table 4.68 - Values for <ReportingUnitType>

\begin{tabular}{|c|c|}
\hline Value & Value Description \\
\hline ballot-batch & Used for reporting batches of ballots that may cross precinct boundaries. \\
\hline ballot-style-area & Used for a ballot style areas generally composed of precincts \\
\hline borough & $\begin{array}{l}\text { Used in CT, NJ, PA, other states, and New York City for boroughs. For AK } \\
\text { and LA, see county. }\end{array}$ \\
\hline city & Used for a city that reports results and/or for the district that encompasses it. \\
\hline city-council & Used for city council districts. \\
\hline combined-precinct & $\begin{array}{l}\text { Used for one or more precincts that have been combined for the purposes of } \\
\text { reporting. Used for "Ward" if "Ward" is used interchangeably with } \\
\text { "CombinedPrecinct". }\end{array}$ \\
\hline congressional & Used for U.S. Congressional districts. \\
\hline county & $\begin{array}{l}\text { Used for a county and/or for the district that encompasses it. In AK, used for } \\
\text { counties that are called boroughs. In LA, used for parishes. }\end{array}$ \\
\hline county-council & Used for county council districts. \\
\hline drop-box & Used for a dropbox for absentee ballots. \\
\hline judicial & Used for judicial districts. \\
\hline municipality & $\begin{array}{l}\text { Used as applicable for various units such as towns, townships, villages that } \\
\text { report votes and/or for the district that encompasses it. }\end{array}$ \\
\hline polling-place & Used for a polling place. \\
\hline precinct & $\begin{array}{l}\text { Used also for "Ward" or "District" when these terms are used } \\
\text { interchangeably with "Precinct". }\end{array}$ \\
\hline school & Used for a school district. \\
\hline special & Used for a special district. \\
\hline split-precinct & Used for splits of precincts. \\
\hline state & Used for a state and/or for the district that encompasses it. \\
\hline state-house & Used for a state house or assembly district. \\
\hline state-senate & Used for a state senate district. \\
\hline town & $\begin{array}{l}\text { Used in some New England states as a type of municipality that reports votes } \\
\text { and/or for the district that encompasses it. }\end{array}$ \\
\hline township & Used in some mid-western states as a type of municipality that reports votes \\
\hline
\end{tabular}

${ }^{6}$ A future version of this schema may specify geographies differently using a structured registry of defined types. 


\begin{tabular}{|c|l|}
\hline Value & \\
\hline & and/or for the district that encompasses it. \\
\hline utility & Used for a utility district. \\
\hline village & $\begin{array}{l}\text { Used as a type of municipality that reports votes and/or for the district that } \\
\text { encompasses it. }\end{array}$ \\
\hline vote-center & Used for a vote center. \\
\hline ward & Used for combinations or groupings of precincts or other units. \\
\hline water & Used for a water district. \\
\hline other & Used for other types of reporting units not included in this enumeration. \\
\hline
\end{tabular}

\section{Schema Definition:}

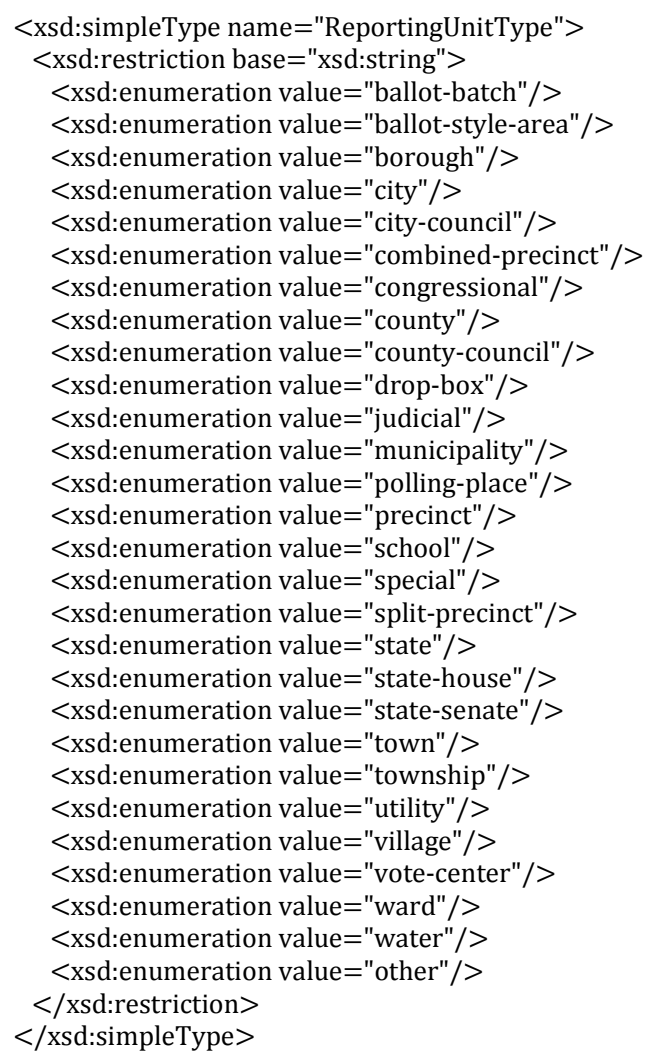




\subsubsection{The ResultsStatus Enumeration}

Enumeration for the status of the election results in the <ElectionReport $>$ element.

Table 4.69 - Values for <ResultsStatus>

\begin{tabular}{|c|l|}
\hline Value & \multicolumn{1}{|c|}{ Value Description } \\
\hline certified & For results that have been certified by the election authority. \\
\hline correction & For results that are a correction to an earlier report. \\
\hline pre-election & For a pre-election data. \\
\hline recount & For results that are a recount of an earlier election. \\
\hline unofficial-complete & $\begin{array}{l}\text { For results that are unofficial and complete, e.g., the complete election } \\
\text { night results. }\end{array}$ \\
\hline unofficial-partial & For results that are unofficial and partial, e.g., partial election night results. \\
\hline
\end{tabular}

\section{Schema Definition:}

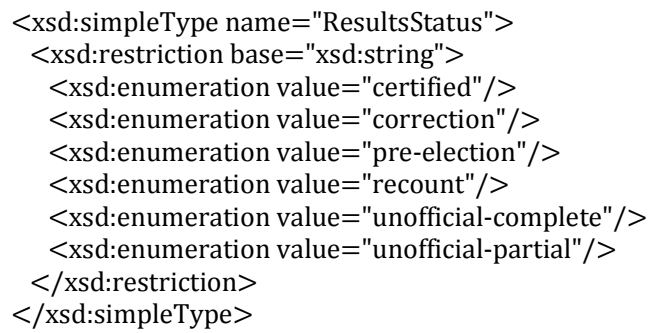




\subsubsection{The VoteVariation Enumeration}

Enumeration for contest algorithm or rules in the <Contest> element.

Table 4.70 - Values for <VoteVariationType>

\begin{tabular}{|c|l|}
\hline Value & \\
\hline 1-of-m & For 1 of M voting. \\
\hline approval & For approval voting. \\
\hline borda & For the Borda count voting. \\
\hline cumulative & For cumulative voting. \\
\hline majority & For majority voting. \\
\hline n-of-m & For N of M voting. \\
\hline plurality & For plurality voting. \\
\hline proportional & For proportional voting. \\
\hline range & For range voting. \\
\hline rcv & For ranked choice voting. \\
\hline super-majority & For super majority voting. \\
\hline other & Used when the vote variation type is not included in this enumeration. \\
\hline
\end{tabular}

\section{Schema Definition:}

\footnotetext{
$<$ xsd:simpleType name $=$ "VoteVariation" $>$ $<$ xsd:restriction base $=$ "xsd:string" $>$ $<$ xsd:enumeration value $=$ "1-of-m" $/>$ $<$ xsd:enumeration value $=$ "approval" $/>$ $<$ xsd:enumeration value $=$ "borda" $/>$ $<$ xsd:enumeration value $=$ "cumulative" $/>$ $<$ xsd:enumeration value $=$ "majority" $/>$ $<$ xsd:enumeration value $=$ "n-of-m" $/>$ $<$ xsd:enumeration value $=$ "plurality" $\mid>$ $<$ xsd:enumeration value $=$ "proportional" $/>$ $<$ xsd:enumeration value $=$ "range" $/>$ $<$ xsd:enumeration value $=$ "rcv" $/>$

$<$ xsd:enumeration value $=$ "super-majority" $/>$

$<$ xsd:enumeration value $=$ "other" $/>$

$</$ xsd:restriction $>$

$</$ xsd:simpleType $>$
} 


\subsection{Primitives}

The following sections deal with primitives in the schema.

\subsubsection{The HTMLColorString Primitive}

For a string containing a 6-digit Red-Green-Blue (RGB) code that can be displayed using HTML. Used in < Party > to associate a web-displayable color with the party. The RGB code is specified in hexadecimal, such that the RGB code for the color green is "00FF00" ("\#00" + “\#FF” + “\#00”).

Schema definition:

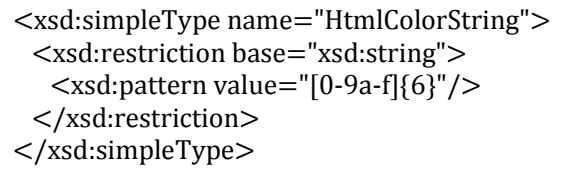

\subsubsection{The Primitive ShortString Primitive}

For defining a 16-character annotation, used with character strings in <AnnotatedString $>$.

Schema definition:

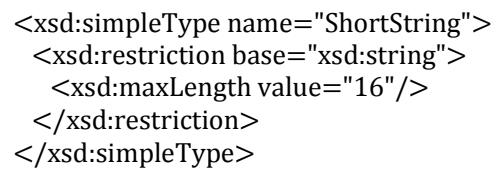

\subsubsection{The Primitive TimeWithZone Primitive}

For defining a time pattern, including the time zone, used in $<$ Hours $>$ for defining a schedule, using the pattern: Hh:mm:ss+05.

Schema definition:

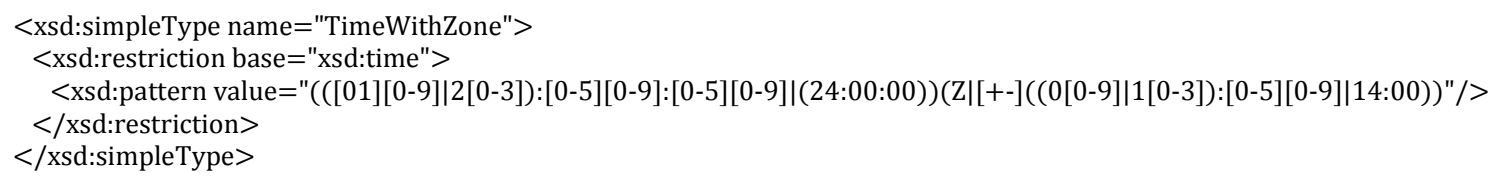




\section{$5 \quad$ XML Schema Aspects and Usage}

This section presents an overview of various aspects and usage of the XML schema, including:

1. General layout of instance files,

2. How UML relationships are implemented in the XML schema,

3. How geopolitical geography is implemented,

4. How contests and ballot selections are implemented,

5. How vote counts and summary vote counts are implemented, and

6. How ballot counts are linked to geopolitical geography

No attempt is made to provide a complete overview of usage, but more to describe in general how most elements are to be used.

\subsection{General Layout of XML Instance Files}

Instance files are built by creating collections of elements such for office descriptions, geopolitical units, candidates, contests, etc., and then linking the elements as necessary using the objectld attributes.

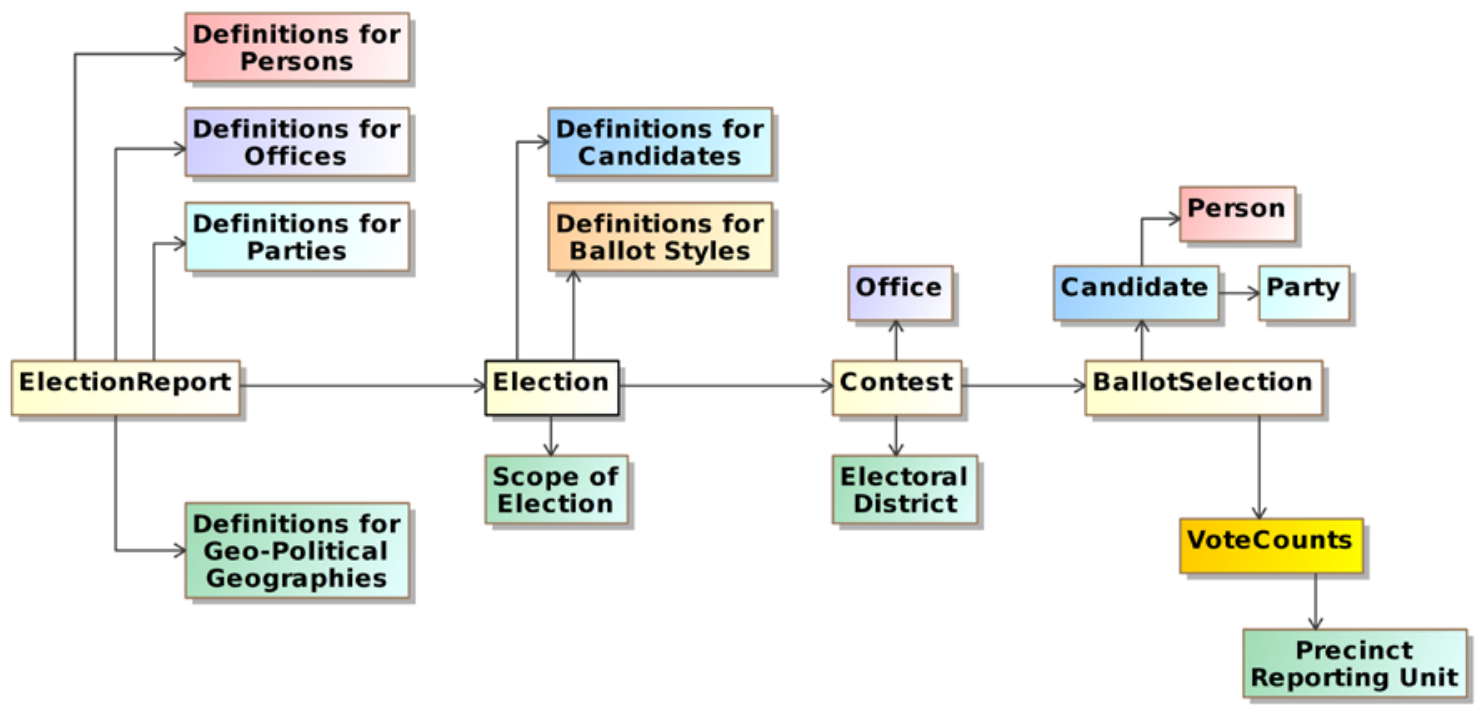

Figure 12 - General Layout of Instance Files

So as to accommodate all use cases (pre-election, election night, and post-election), many elements in the schema are optional, thus instance files will vary in content but generally follow the same structure as shown in Figure 12 and listed below:

- <ElectionReport> element, containing:

o <Election> element(s);

o <GpUnit> elements; 


\author{
$0<$ office $>$ and <OfficeGroup $>$ elements; \\ $0 \quad<$ Party $>$ elements; and \\ o $<$ Person $>$ elements; \\ - $\quad<$ Election> element(s), containing: \\ o <BallotStyle> elements; \\ o <Candidate> elements; \\ $0<$ Contest $>$ element for Contest 1: \\ - $\quad<$ BallotSelection > elements for the ballot selections in Contest 1; \\ - <VoteCounts > elements associated with the ballot selections: \\ - <GpUnit> elements for associating vote counts with precincts; \\ $0<$ Contest $>$ element for Contest 2, etc.
}

Many of the elements make use of enumerated values, for example, the <CountStatus $>$ element makes use of the <CountItemType > enumeration for filtering vote counts by ballot type. Most enumerations include a value of other for use when none of the enumerated values is applicable, and the elements using these enumerations include an <otherType > element that can be used to hold another value.

\title{
5.1.1 Wrapper Elements
}

Collections of elements in <ElectionReport $>$ and <Election $>$ that are likely to be quite large are wrapped by container elements so as to make instance files easier to read in XML viewers. For example, in <Election >, <Contest $>$ is wrapped by <ContestCollection $>$, which then contains 1 or more <Contest $>$ sub-elements, as follows:

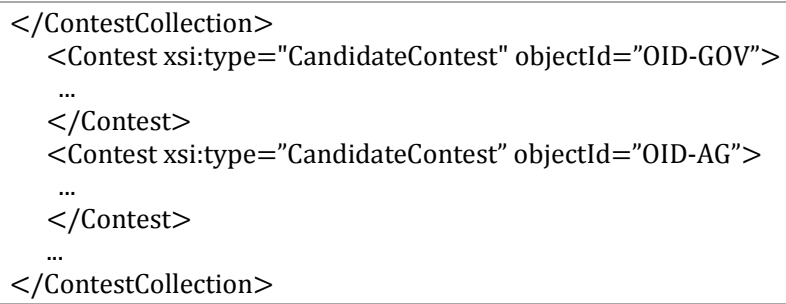

Figure 13 - Use of Wrapper Elements

This applies as well to $<$ BallotStyle $>$, <Candidate $>$, $<$ GpUnit $>,<$ Office $>$ and $<$ OfficeGroup $>$, $<$ Party $>$, and $<$ Person $>$. Additionally, inside $<$ BallotSelection $>$, $<$ VoteCounts $>$ is wrapped by $<$ VoteCountsCollection $>$.

\subsubsection{How UML Relationships Are Implemented in the XML Schema}

The XML schema is generated from the UML model, thus having some understanding of how the XML is generated will assist users of the schema as well as others who may wish to extend the model or schema for other purposes. This section provides a brief overview of the schema generation. 
When XML is generated from the UML model, the classes in the model get generated as major XML elements, e.g., the UML Candidate class results in the XML <Candidate> element. The attributes in the UML classes get generated as XML sub-elements within the XML major element, e.g., the UML attribute Name in the Candidate class gets generated as the sub-element $<$ Name $>$ in the $<$ Candidate $>$ major element.

The schema treats major elements such as candidates or contests or parties as if they are tables in a database that are linked together depending on the type of relationship between the objects. The relationships between UML classes result in XML elements that function differently depending upon the type of relationship. As a brief guide, using Figure 8, the directed composition relationship between Election and Contest results in one or more XML subelements named < <ontest $>$ being created in <Election $>$.

$<$ Contest xsi:type="CandidateContest" objectId="OID-AG" $>$

$<$ Name $>$ Attorney General $</$ Name $>$

$</$ Contest $>$

Figure 14 - "Is a Type of" Relationship in XML

"Is a Type of” or "Instance of” relationships are slightly more complex. For Contest and CandidateContest in Figure 8, CandidateContest is a type of Contest and thus is generated with $<$ Contest $>$ as an XML extension base and all attributes and elements of <Contest $>$ are global to elements that use <contest $>$ as an extension base. The XML syntax for using $<$ contest $>$ for a candidate contest is shown in Figure 14.

"Is a Type of" relationships are used in cases such as < Contest> where there can be several types of contests, but the different types of contests generally require the same set of attributes plus additional attributes that are specific to a type. Thus, <contest $>$ is generated as an abstract XML element with all of its sub-elements and attributes global to the specific types of contests, e.g., CandidateContest. But, the sub-elements of CandidateContest are private to CandidateContest.

\subsubsection{Directed Association Relationships}

Directed association relationships require more explanation. Directed association relationships are used throughout the UML model and in the XML schema to reference or link one element to another, and they give XML instance files the capability to be structured and used as tables in a database, with pointers in one table referencing other tables as needed. An example of this is the $<$ PartyId $>$ sub-element in the <Candidate $>$ element, which serves to link a candidate with the candidate's party.

First, when the XML schema is generated, every class in the UML model that is at the endpoint of a directed association relationship is generated with an objectId attribute of type $x s d: I D$. For example, $<$ Party $>$ is referenced from a number of classes, thus the $<$ Party $>$ XML element is generated with the objectId attribute. Type $x$ sd:ID requires that objectId be a unique legal XML value that does not contain a colon, and digits, the hyphen, and the period may not be used as the starting character. 
Secondly, for the class at the starting point of the directed association, an element is generated that serves to reference the element at the endpoint. Furthermore, the name of the element is the name of the class at the endpoint with "Id" concatenated to it.

For Candidate and Party from Figure 8, the directed association relationship results in an XML sub-element named <PartyId $>$ of type $x$ sd:IDREF being created for $<$ Candidate $>$. The $<$ PartyId $>$ sub-element links to a specific <Party $>$ element that will be associated with that candidate, thus linking the candidate to the candidate's party. The usage is shown below:

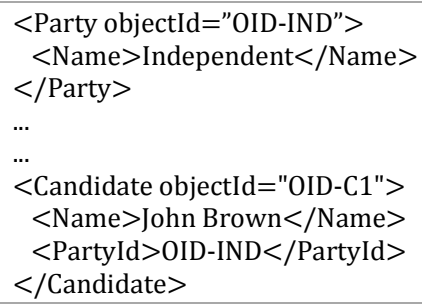

Figure 15 - Directed Association in XML

In this example, "OID-IND" is the value of the objectId attribute for the <Party> element defined for the Independent Party. To link candidate John Brown to this <Party > element, the corresponding <Candidate> element uses < PartyId> and the same "OID-IND” value.

\subsection{Defining Geopolitical Geography}

A primary feature of the schema is the geopolitical geography element <GpUnit>, which can be used in a variety of ways to exactly mirror a jurisdiction's geopolitical geography. Vote counts in the real world are reported by geopolitical geographies, thus <GpUnit> elements are used in an election report to list the geopolitical units of geography that lay within the geographic scope of the election results such as counties, cities, precincts, districts, etc., so that ballot and vote counts can be associated with them. The UML model and XML schema permit counts to be reported at a high aggregation level only if desired, thus < GpUnit> elements are required in two places:

1. The <Contest $>$ element contains a required link to a $<$ GpUnit $>$ defined for the electoral district of the contest, so that vote counts can be linked to that <GpUnit> element. The $<$ ElectoralDistrictId $>$ sub-element is used to link to the <GpUnit> element defined for the electoral district.

2. The <Election > element contains a required link to a $<$ GpUnit $>$ defined for the geographical scope of the election, again using the <ElectionScopeId > sub-element.

If counts need to be associated with the smaller geographies, i.e., precincts, additional <GpUnit> elements need to be defined for these geographies. <GpUnit> elements, as described in section 4.2.14, can be used for the following major types of geographies: 
- Geographies that report election results or that can have vote counts associated with them, including for states, counties, cities, towns, precincts, split precincts, ballot style areas, etc.;

- Geographies that serve as electoral districts, i.e., that have contests associated with them;

- Geographies that serve as districts that have no contests associated (e.g., tax districts); and

- Geographies that are for specific locations or specific objects including:

o Voting devices;

o Polling places or vote centers;

o Drop boxes; and

o Other miscellaneous objects.

Additionally, <GpUnit> elements can be defined for other types of objects not listed in the $<$ ReportingUnitType $>$ enumeration by using other in the <Type > sub-element and then listing the other type of object in the <OtherType > sub-element.

\subsubsection{Defining <GpUnit> Elements for Governmental and Administrative-based Geographies}

Figure $16^{7}$ shows an example of geopolitical geography in the town of Richmond, WI. Figure 17 shows the <GpUnit> definitions for the governmental and administrative-based geographies (in Wisconsin, a ward is the same as a precinct). The assumption here is that the election results will be reported by these geographies (county, town, and ward), thus accordingly there are <GpUnit> definitions for these geographies. The county needs to be linked with the state, the town needs to be linked with the county, the combined wards need to be linked with the town, the five precincts need to be linked with their parent combined precincts, and the two split precinct elements need to be linked with their parent Ward 1 precinct. Thus, the <GpUnit> definitions for the parent geographies include <ComposingGpUnitIds $>$ to reference the geographies that are contained within the parent elements.

\footnotetext{
${ }^{7}$ This figure is repeated from Figure 7.
} 


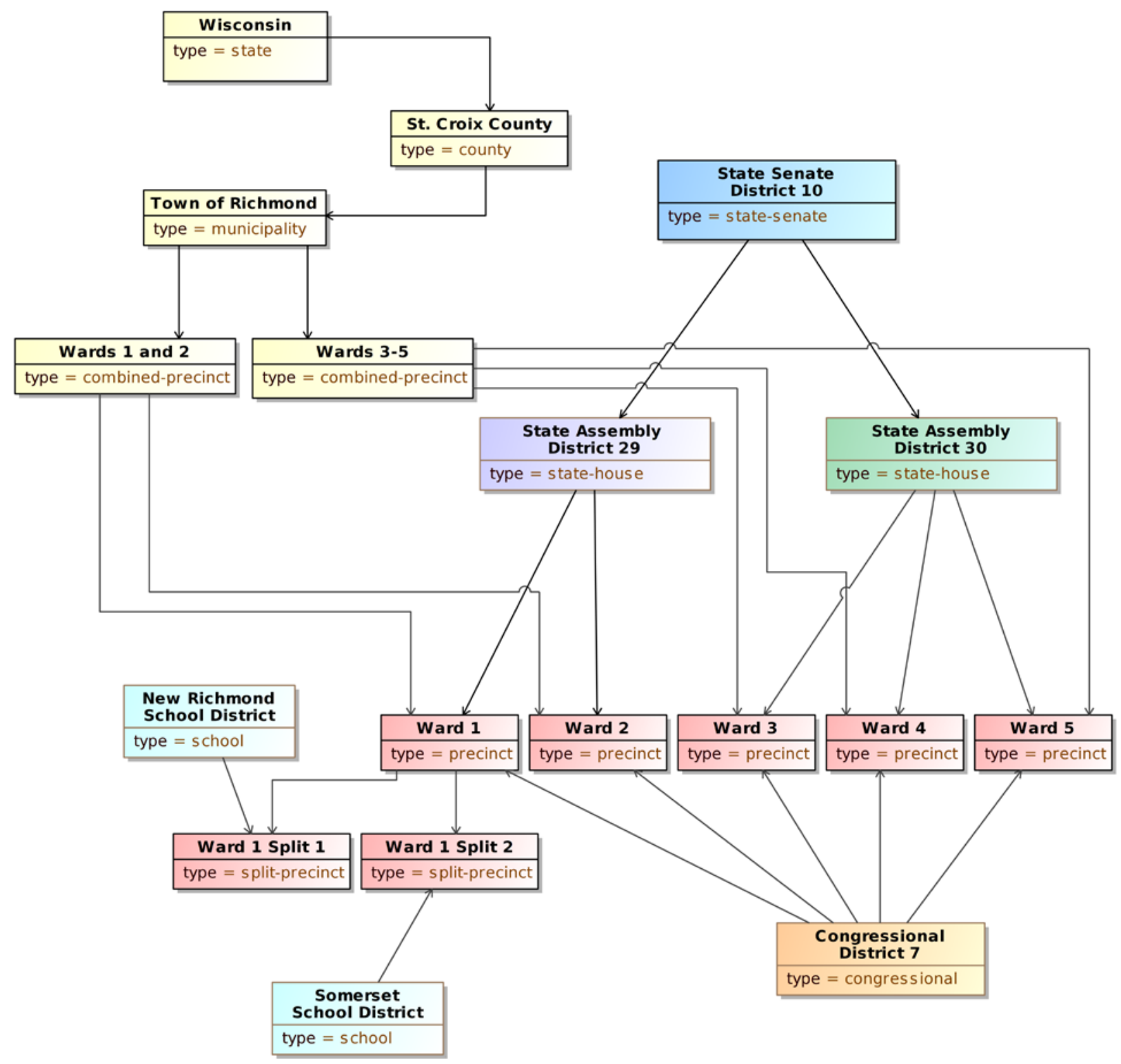

Figure 16 - Geopolitical Geography Example from Wisconsin

However, there is a rule that must be observed when defining these relationships:

A $<$ GpUnit $>$ element's $<$ ComposingGpUnitIds $>$ element must only reference those geopolitical units that are wholly contained within the scope of the $<G p U n i t>$ element.

Accordingly, split precincts are wholly contained within precincts, thus the proper way to structure the associated <GpUnit> elements is for the precinct element to contain a $<$ ComposingGpUnitIds > element that references the split precinct elements - and not vice versa. If this rule is violated, an application would not be able to find information correctly within the instance file. Figure 18 shows a hierarchy of containment relationships, with precincts and split precincts being the basic foundational bricks for all the geographies. 


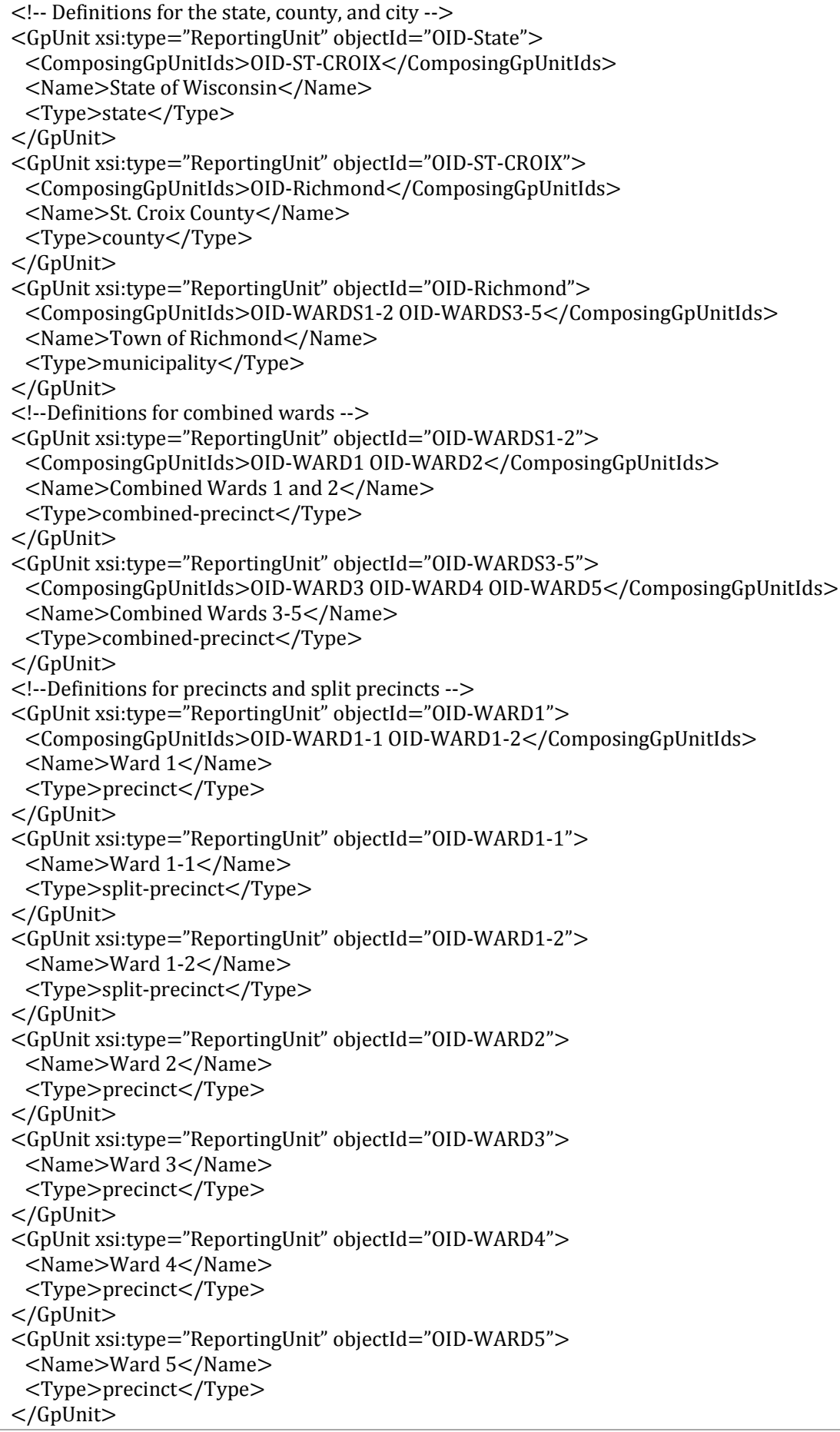

Figure 17 - <GpUnit> Definitions for Governmental and Administrative Geographies 


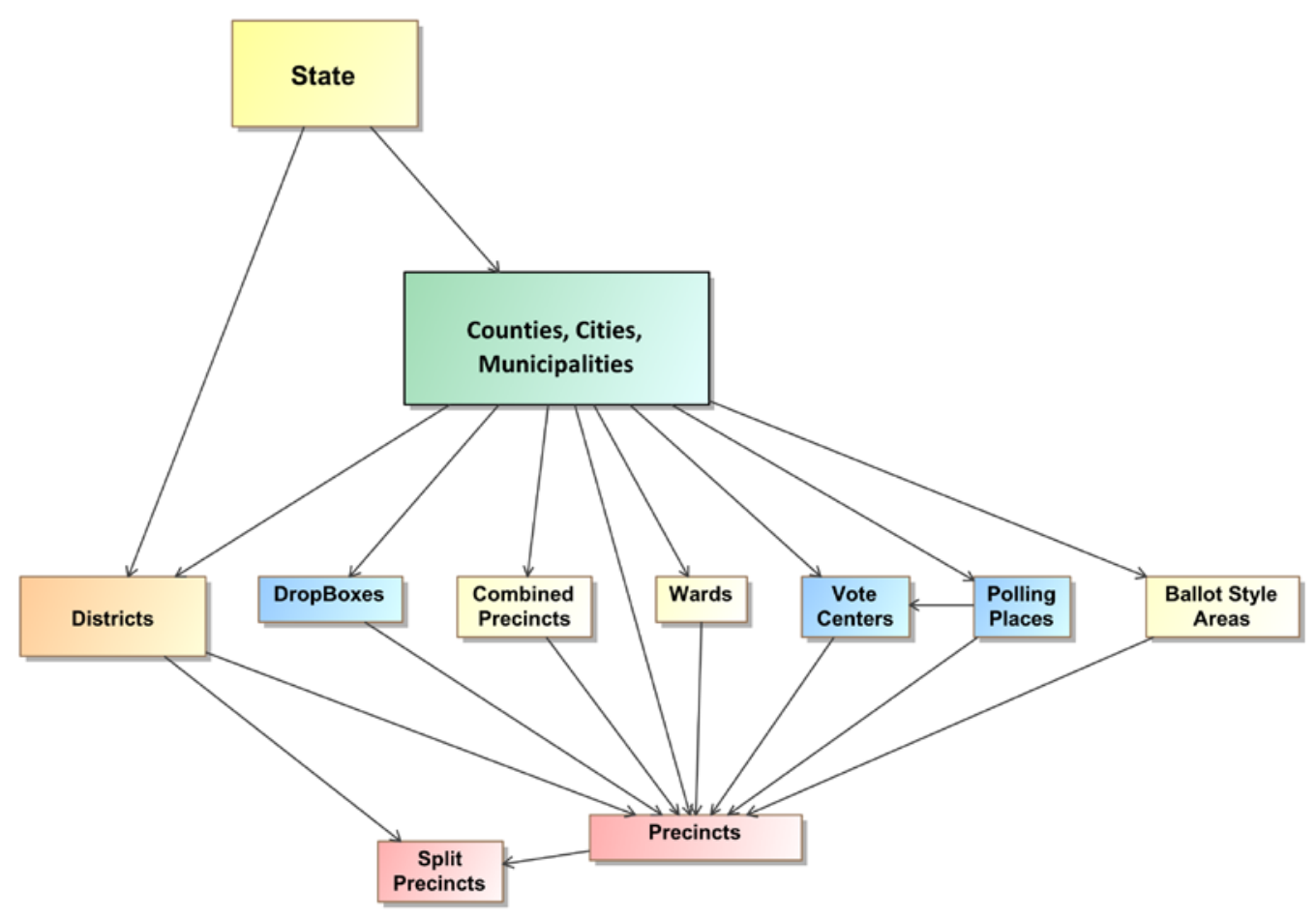

Figure 18 - Containment Relationships

\subsubsection{Associating an Election Authority with the <GpUnit> Element Defined for the Scope of the Election}

The <Election> element references a <GpUnit $>$ element defined for the scope of the election, and that <GpUnit> element can then include the <ElectionAdministration > element for associating election authority information. <ElectionAdministration> includes <ContactInformation $>$ for the election authority and, using <ElectionOfficialPersonIds $>$, references one or more $<$ Person $>$ elements defined for individuals/organizations associated with the election authority.

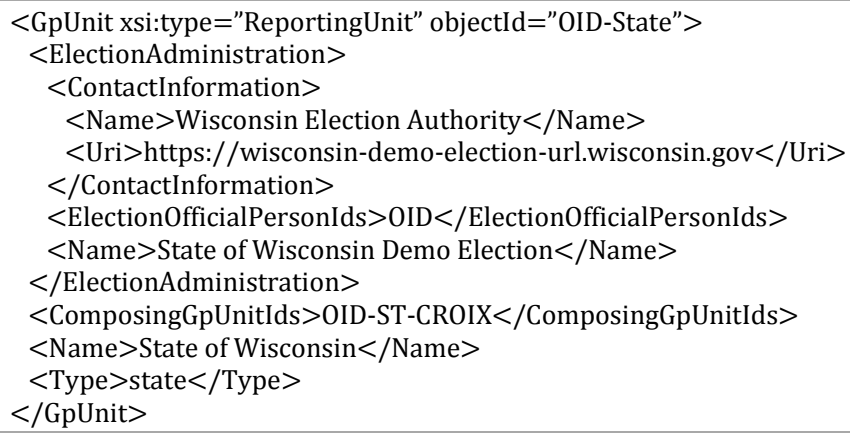

Figure 19 - Associating Election Authority Information

In Figure 16, the State of Wisconsin is assumed as the scope of the election, and in Figure 17, a $<$ GpUnit> element is defined for the state. Figure 19 shows the definition for the state, but this time with the election authority information added. 


\subsubsection{Defining <GpUnit> Elements for Political-based Geographies}

Figure 20 shows the districts and the wards that compose them from Figure 16. Figure 21 shows the <GpUnit $>$ definitions for the districts as well as the use of <ComposingGpUnitIds $>$ elements to link the districts to the wards that compose them.

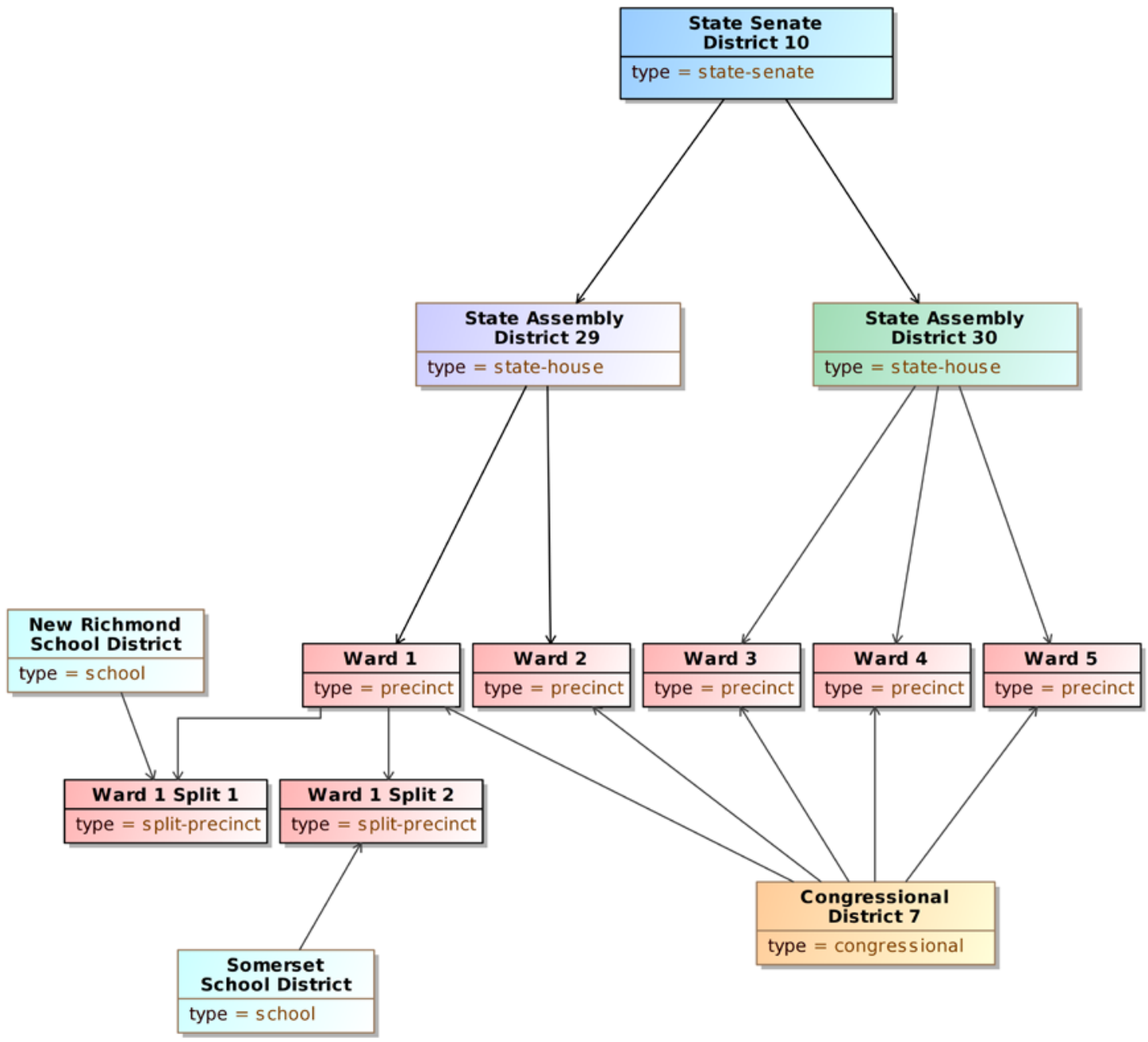

Figure 20 - District <GpUnit> Elements

The <IsDistricted $>$ boolean is used in this example. It is not strictly necessary, as it is possible to identify districts by their type or by examining the <Contest $>$ element's < ElectoralDistrictId > subelement, which links to the electoral district associated with the contest (see section 4.2.6). However, if a district is defined but is not linked from a contest, or if the type of district is not listed in the <ReportingUnitType $>$ enumeration and therefore <OtherType $>$ is used, then $<$ IsDistricted $>$ is necessary to identify the <GpUnit $>$ as a district. The <IsDistricted $>$ boolean can also be used to signify that a <GpUnit> defined as a jurisdiction, e.g., a county, is also used as a district for, e.g., county-wide contests. 


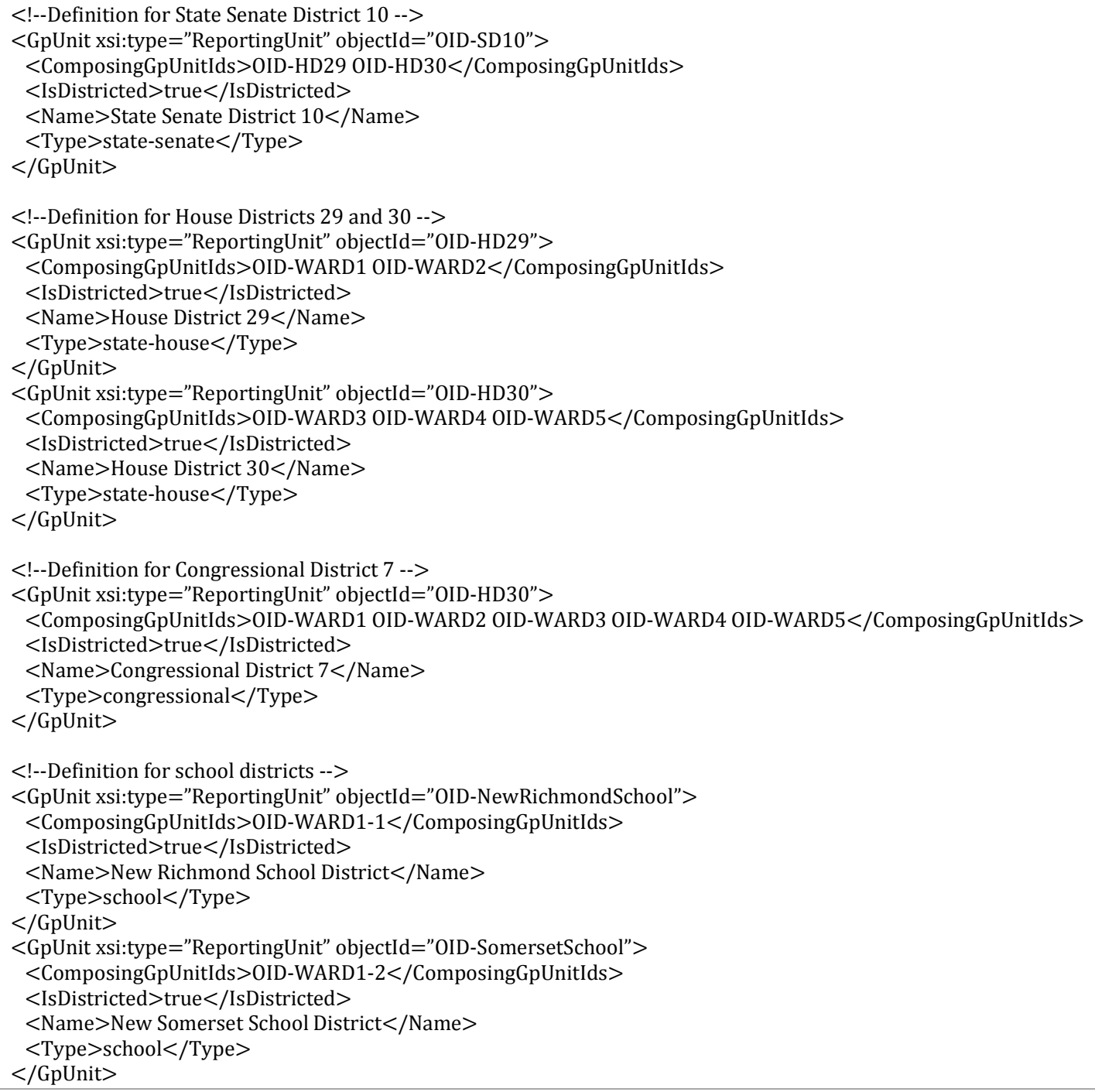

Figure 21 - Defining Political Geographies and Linking to Precincts

\subsubsection{Dealing with Duplicate Electoral Districts}

There are, quite often, multiple jurisdictional-wide contests that use, for their respective electoral districts, the same physical geography. In a given county, for example, contests for county executive and at large county council positions will all use the county as the electoral district. Because <GpUnit> elements defined for these geographies may have very large $<$ ComposingGpUnitIds > sub-elements, instance files could grow unnecessarily large if different but essentially duplicate <GpUnit> elements are defined for each of the contests that share what is essentially the same electoral district. 


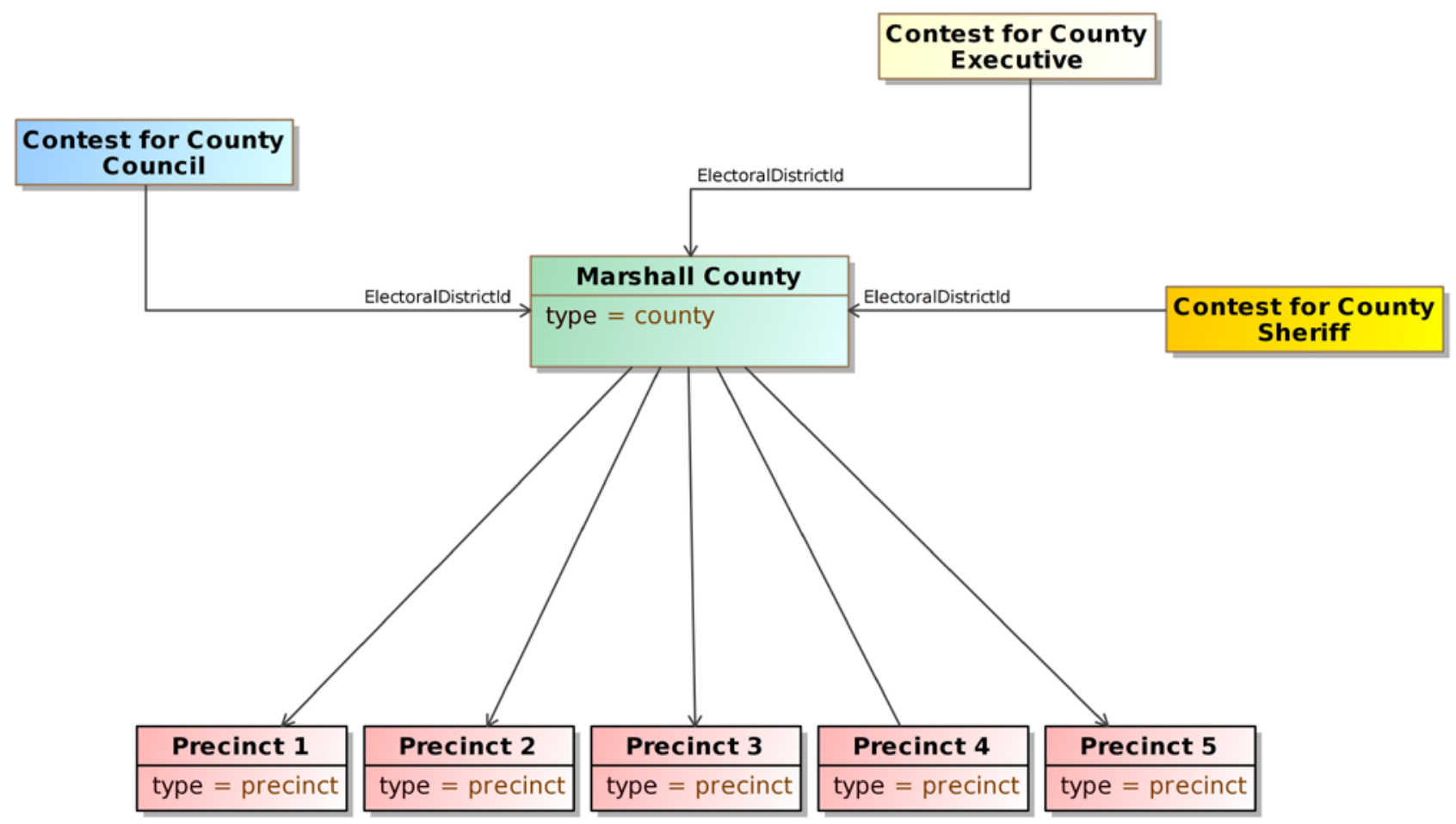

Figure 22 - Re-Using Electoral District Links

An option for reducing the file size is to re-use the <GpUnit> element defined for the, say, county. Provided that this element is linked to its composing precincts, the contests that are county-wide can use this same <GpUnit> element for the electoral district. The generating application could, effectively, keep track of the objectId attribute of the county <GpUnit> element and then use the associated value for each contest's < ElectoralDistrictId > sub-element. This is shown pictorially in Figure 22.

Thus, if all contest definitions for jurisdictional-wide contests use the same value for the $<$ ElectoralDistrictId > sub-element, duplication can be reduced and file sizes may be smaller, especially for larger jurisdictions.

\subsection{Defining Contests}

After the <GpUnit> elements have been defined, contests can then be defined using the <Contest> element. Defining contests involves the use of several elements, depending on what type of contest is involved and whether and what types of vote counts are desired. The elements needed for contest definitions are as follows:

1. The <Contest $>$ abstract element, to identify the name of the contest and other attributes and its type:

a. CandidateContest, for a contest involving candidates;

b. BallotMeasureContest, for a contest involving a ballot measure;

c. PartyContest, for a contest involving a straight party selection; and 
d. RetentionContest, for a contest involving a judicial or other retention;

2. The <BallotSelection > abstract element, to identify a selection on the ballot for that corresponding contest and its type:

a. CandidateSelection, if the selection is for a candidate;

b. BallotMeasureSelection, if the selection is for a ballot measure or retention; and

c. PartySelection, if the selection is for a straight party selection;

3. Within <BallotSelection $>$, <VoteCounts $>$ to report the number of votes for the ballot selection, for the contest as a whole or broken out by, e.g., precincts and/or types of ballots;

4. A link to a GpUnit element defined for the contest's electoral district, using the $<$ ElectoralDistrictId > sub-element; and

5. The <SummaryCounts > element, to report summary counts for the contest, e.g., total ballots cast, overvotes, undervotes, etc.

It is possible to define contests without associated $<$ BallotSelection $>$ elements as well as without any of the elements for election results; but these elements are required if associating vote counts with ballot selections.

CandidateContest is the type of contest likely to be used most often; it is for contests involving candidates and has several elements associated with it including $\langle$ PrimaryPartyIds $\rangle$, a link to the political party used when a primary election, $<$ NumberElected $>$, for the number of seats associated with the office and <VotesAllowed $>$, for the number of selections a voter can make in the contest.

PartyContest is included as a contest type because a number of states keep track of the number of straight party selections made by voters.

Following is a short example of a contest involving CandidateContest that illustrates using ballot selections and counts, with the counts being aggregated, that is, associated with the electoral district of the contest as a whole:

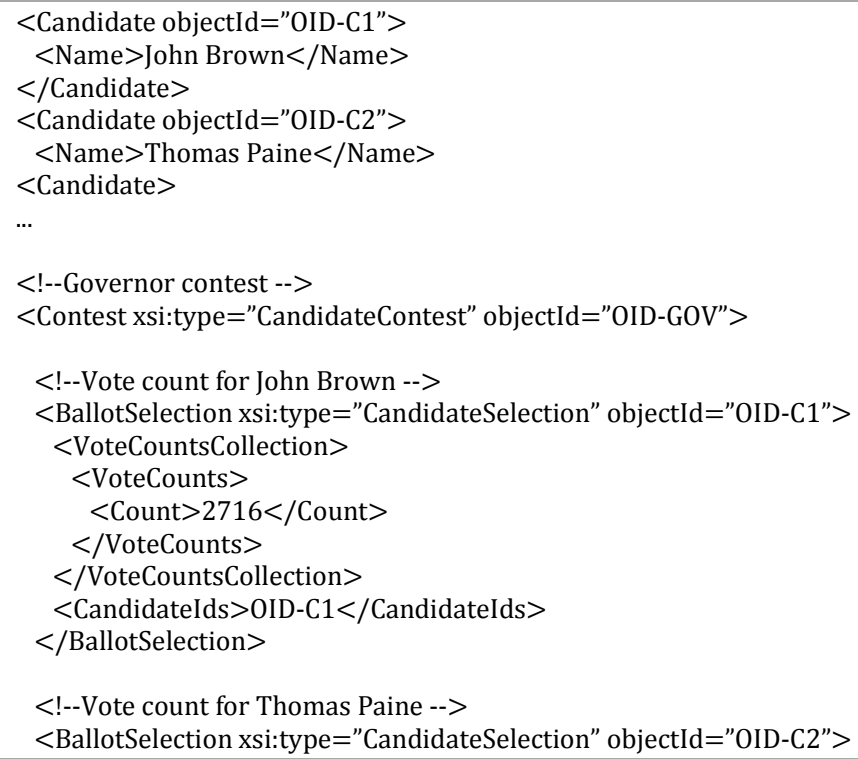


$<$ VoteCountsCollection $>$

$<$ VoteCounts $>$

$<$ Count $>2392</$ Count $>$

$</$ VoteCounts $>$

$</$ VoteCountsCollection $>$

$<$ CandidateIds $>$ OID-C $2</$ CandidateIds $>$

$<$ BallotSelection $>$

$<$ ElectoralDistrictId $>$ OID-State $<$ /ElectoralDistrictId $>$

$<$ Name $>$ Governor $</$ Name $>$

$<$ VotesAllowed $>1<$ /VotesAllowed $>$

$</$ Contest $>$

Figure 23 - Basic Contest Definition and Linkage to Vote Counts

Contests for BallotMeasureContest and PartyContest are implemented as per this example.

\subsection{Associating Vote Counts with Contests}

The XML schema includes the capability to associate vote counts with contests in a variety ways, including the following:

1. As aggregated vote counts (applying to the entire electoral district):

- The vote counts can be broken out (or filtered by) ballot type, device type, and by specific device and manufacturer;

2. As subsets of vote counts, each associated with specific geopolitical units within the electoral district such as precincts or split precincts:

- The vote counts can be broken out by ballot type, device type, and by specific device and manufacturer;

3. As summary vote counts that can be aggregated as well as associated with specific geopolitical units:

- The summaries can be broken out by ballot type, device type, and by specific device and manufacturer; and

- The summaries can include:

o Total number of ballots cast for the contest;

o Total number of overvotes and undervotes in the contest; and

o Total number of write-ins in the contest.

The following sections go into more detail on how to report counts accordingly.

\subsubsection{Aggregated Vote Counts}

The previous section showed an example of basic contest definition involving aggregated vote counts applying to the entire electoral district. To go into greater level of detail and report the aggregated counts by ballot type or device type, one includes additional values from the $<$ CountItemType $>$ or < DeviceType $>$ enumerations. These enumerations specify different ballot types or device items that can be used as, essentially, filters on the aggregated results. An 
example of this is as follows, using the contest for governor and values from the $<$ CountItemType > enumeration:

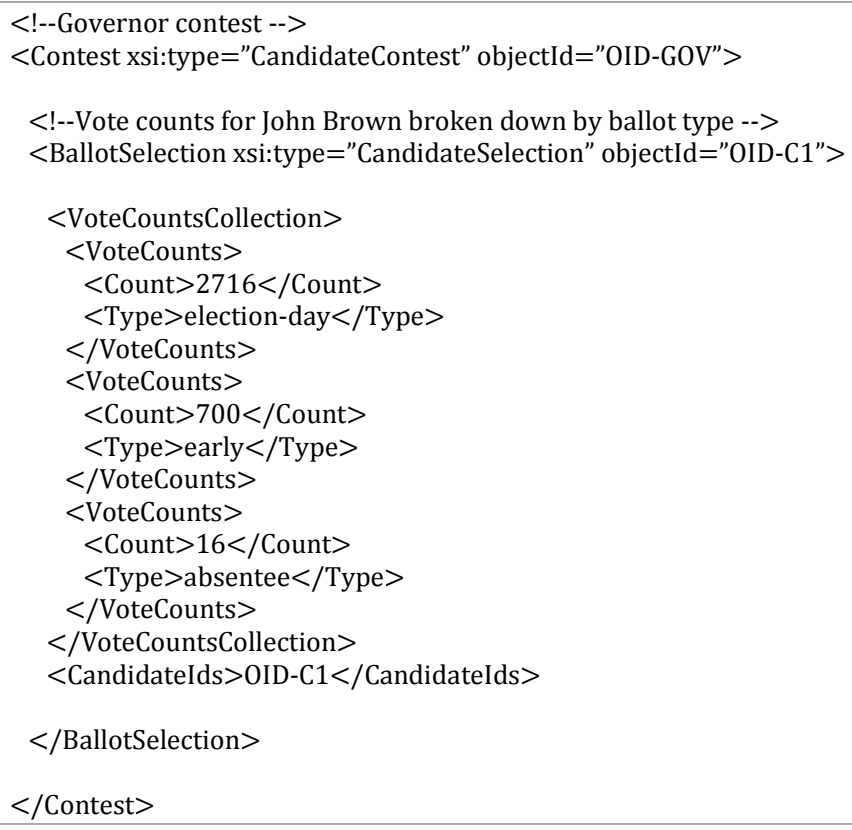

Figure 24 - Linking Aggregated Vote Counts to Contests

This example answers the questions, how many votes did John Brown get for the contest as a whole, filtered by ballots cast on election day, by ballots cast during the early voting period, or by absentee ballots.

\subsubsection{Vote Counts by Precinct, Ballot, and Device Type}

For associating vote counts broken down by geopolitical units such as precincts and split precincts, one uses the <GpUnitId > sub-element to link the counts to <GpUnit> elements defined for, the precincts and split precincts. For example, to associate the vote count for John Brown by ward in Figure 16, the XML is:

$<$---Governor contest -->

$<$ Contest xsi:type="CandidateContest" objectId="OID-GOV">

$<$ !--Vote count for John Brown broken down by ward -->

$<$ BallotSelection xsi:type="CandidateSelection" objectId="OID-C1">

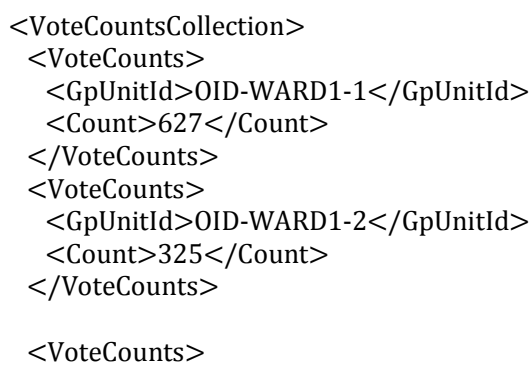


$<$ GpUnitId $>$ OID-WARD2 $</$ GpUnitId $>$

$<$ Count $>432<$ /Count $>$

$</$ VoteCounts $>$

$</$ VoteCountsCollection $>$

$<$ CandidateId $>$ OID-C $1</$ CandidateId $>$

$</$ BallotSelection $>$

Figure 25 - Linking Vote Counts to Precincts

In this example, note that the vote counts for John Brown are broken down, in Ward 1, for Ward 1's splits (as opposed to breaking it down by Ward 1 as a whole). Since all of Ward 1 is in the electoral district for the Governor's contest, it is not necessary to break down the vote count by the split precincts of Ward 1, but doing so would answer questions such as, "How did the voters in the New Richmond or Somerset school districts vote in the Governor’s contest?”

To break the vote counts down further by ballot type or device type, one again uses values from the <CountItemType > or <DeviceType> enumerations. Using Ward 2 from the previous example, the XML is:

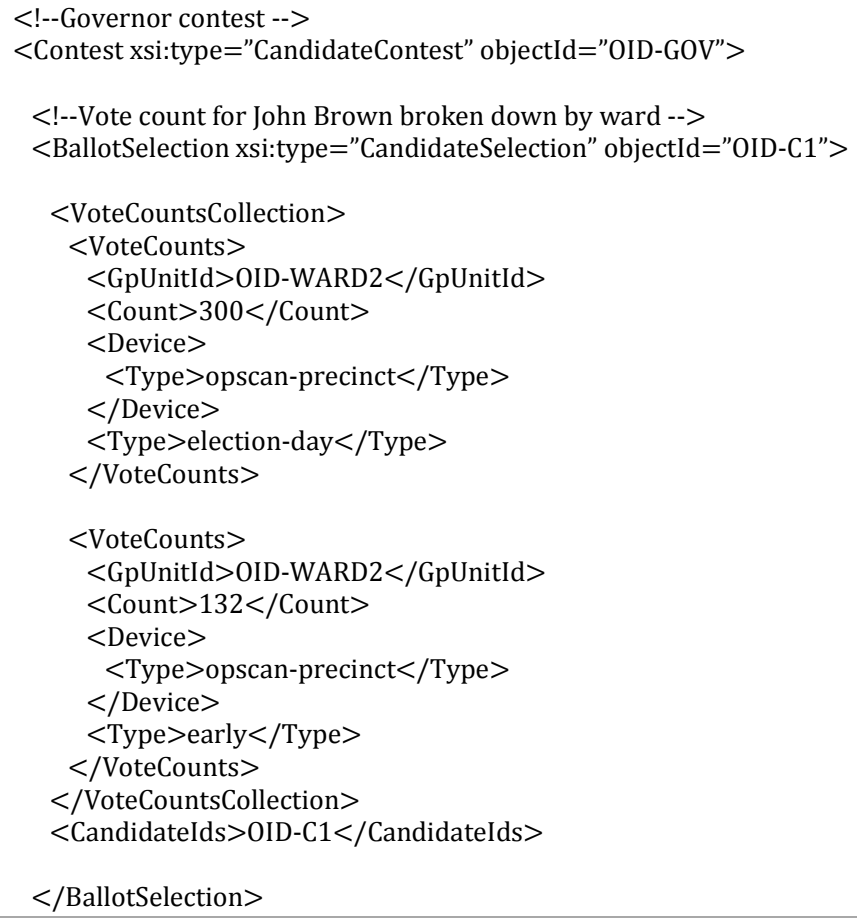

Figure 26 - Filtering Vote Counts by Device and Ballot Type

Thus, using the two examples, John Brown received 300 votes in Ward 1 on election day using precinct-count optical scanner. He received 132 votes during the early voting period, again using a precinct-count optical scanner. 


\subsubsection{Summary Vote and Ballot Counts}

It is possible to associate summaries of vote and ballot counts for the contest for various purposes including for reconciliations and checks on the contest. These summaries include:

- Total number of ballots cast for the contest,

- Total number of overvotes and undervotes in the contest, and

- Total number of write-ins in the contest.

As with previous examples, it is possible to further filter these counts by ballot type and device type, using values from the <CountItemType $>$ or $<$ DeviceType $>$ enumerations. It is also possible to link the counts to specific geopolitical units such as precincts and split precincts by including the $<$ GpUnitId $>$ sub-element to link the counts to <GpUnit $>$ elements defined for, the precincts and split precincts.

Following is an example of summary counts for a contest using SummaryCounts to report summary vote counts by ballot type and further broken down by ward:

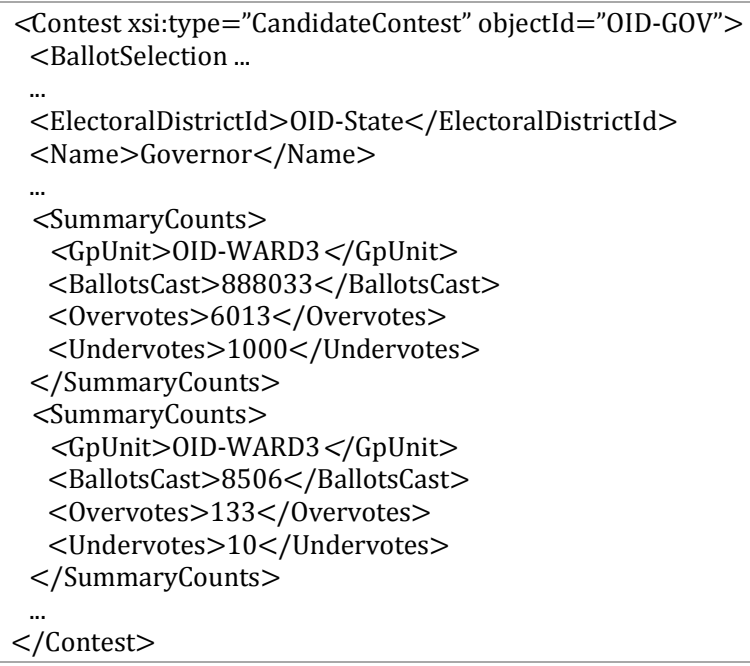

Figure 27 - Associating Summary Counts with Contests

Some jurisdictions report items such as overvotes and undervotes as candidates in a contest as opposed to reporting them separately as a summary of counts. This schema does not prevent one from treating overvotes, undervotes, or other types of counts as candidates in a contest, thus one would report their specific vote counts as shown in section 5.4.1.

\subsection{Associating Summary Ballot Counts with Geopolitical Units}

Lastly, it is possible to associate the ballots cast in each geopolitical unit and filter them by ballot types and device types by, again, including the < summaryCounts $>$ element. The summary counts include: 
- Total number of ballots cast at that geopolitical unit,

- Total number of overvotes and undervotes on all ballots cast at that geopolitical unit,

- Total number of write-ins on all ballots cast at that geopolitical unit, and

- Summary ballot counts for the geopolitical unit broken out by:

o Type of ballot,

o Type of device, and

o Specific device serial number and manufacturer.

Thus, one can associate summary counts for each precinct, etc., and, as possible, go down to the level of specific devices. To do this, the <SummaryCounts $>$ element needs to be added to each $<$ GpUnit> definition, as follows:

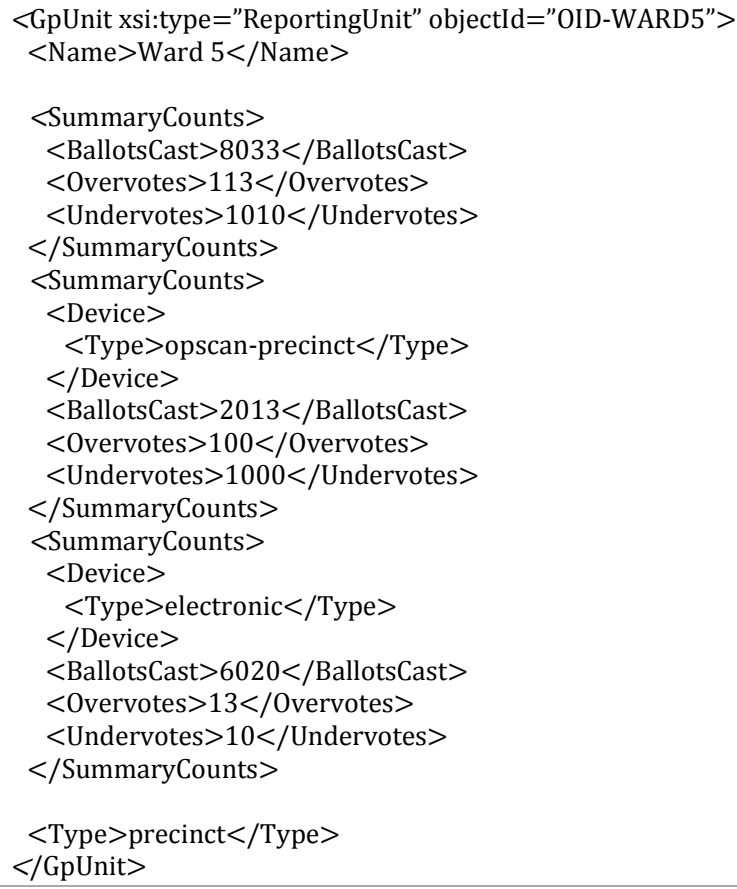

Figure 28 - Associating Summary Ballot Counts with $<$ GpUnit $>$ Elements

In this example, Ward 5 had a total of 8033 ballots cast, with 113 overvotes and 1010 undervotes on all ballots. The count is then broken down by device type, with 2013 ballots cast on precinct optical scan and 6020 ballots cast on an all-electronic device such as a DRE. 


\section{Appendix A-Acronyms}

Selected acronyms and abbreviations used in this document are defined below.

CDF Common Data Format

DRE Direct Record Electronic

EAC Election Assistance Commission

EAVS EAC Election Administration and Voting Survey

EMS Election Management System

FIPS $\quad$ Federal Information Processing Standard

FWAB Federal Write-in Absentee Ballot

JSON JavaScript Object Notation

NIST National Institute of Standards and Technology

OCD-ID Open Civic Data Identifiers

OASIS Organization for the Advancement of Structured Information Standards

SP Special Publication

UML Unified Modeling Language

UOCAVA Uniform and Overseas Citizens Assistance in Voting Act

VIP Voting Information Project

VVSG Voluntary Voting Systems Guidelines

XML eXtensible Markup Language 


\section{Appendix B-Glossary}

Selected terms used throughout this document are defined below. In some of the definitions, there is ancillary information that is not part of the definition but helpful in understanding the definition; this ancillary information is preceded with "Note:”. Synonyms are preceded with "Syn:".

Absentee ballot:

Absentee voting:

Affiliation:

Ballot measure:

Ballot rotation:

Ballot style:

Borough:

Closed primary:

Combined precinct:

Contest:
Ballot resulting from absentee voting.

Voting that can occur unsupervised at a location chosen by the voter and usually done ahead of election day. Note: In some jurisdictions, absentee voting is also called early voting and vote by mail.

Association with a political party. See also: endorsement. Note: Affiliation with a political party does not imply endorsement by that political party; endorsement does not imply affiliation.

Contest in which the choices are typically Yes and No. Syn: referendum.

The process of varying the order of the contest choices within a given contest.

The list of contests and candidates associated with a particular ballot and its associated precinct or split precinct (and party, for some primaries), including language used and the ordering of contests and candidates. Note: In closed primaries, the same collection of ballot style layouts are used within the same ballot style geography, with the specific ballot style layout depending on the voter's affiliation.

Term to signify a subdivision generally smaller than cities in terms of both geographic area and population and administered through a borough code in states such as CN, NJ, PA, and other states. Used to mean a county in AK. Used in New York City to subdivide the city much as counties subdivide a state.

Primary election in which only voters registered with a political party may vote in those party-specific contests associated with that party.

Two or more precincts assigned the same polling place. Syn: consolidated precinct.

A single decision being put before the voters (e.g., the selection of candidates to fill a particular public office or the approval or disapproval of a constitutional amendment). 
Cross-party endorsement: Endorsement of a given contest choice by two or more political parties.

Direct record electronic (DRE):

\section{Early voting:}

Election certification:

Election day:

Election night:

Election management system (EMS):

Election official:

Electoral District:
An electronic vote-capture device that provides choices visible to the voter on a front panel of the machine in which voters directly enter choices into electronic storage with the use of a touch-screen, pushbuttons, or similar device. Note: An alphabetic keyboard is often provided with the entry device to allow for the possibility of write-in votes. The voter's choices are stored in these machines and added to the choices of all other voters.

Voting that occurs prior to election day at a polling location under the supervision of poll workers or election administrative staff. See also: in-person voting. Note: some jurisdictions, early voting is referred to as in-person absentee voting.

The process of certifying the results of an election, including absentee or early votes, votes cast on election day, and valid provisional ballots.

The date on which the election is considered held. Absentee votes and early votes may be cast in advance of election day. Note: Some jurisdictions have an election period of several days or weeks for inperson or remote voting, and there is no one day that is election day.

The period of time starting when the polls close in a jurisdiction in which results may first begin to be reported and ending when all precincts have reported.

Computer systems used to perform such tasks as preparing ballots, setting up tally systems, maintaining voter registration information, generating reports, and to consolidate, report, and display election results. Note: This device receives results data from the vote-capture devices or by manual input, accumulates the results, and reports the accumulated results.

Any county clerk and recorder, election judge, member of a canvassing board, member of a board of county commissioners, member or secretary of a board of directors authorized to conduct public elections, representative of a governing body, or other person contracting for or engaged in the performance of election duties as required by the election code.

As used in elections, administrative divisions in which voters are entitled to vote in contests that are specific to that division, such as those for state senators and delegates. 
Endorsement:

General election:

In-person voting:

Jurisdiction:

Municipality:

N-of-M:

Nonpartisan primary:

Open primary:
Approval by a political party (e.g., as the candidate that the party elects to field in a particular contest and/or as the candidate that should receive straight party votes). See also: affiliation. Note: In some states, more than one party may endorse a contest selection.

Election in which the candidates for contests and offices have generally been chosen during a primary election. Note: In presidential systems, the term refers to a regularly scheduled election where the president, and either "a class" of or all members of the national legislature are elected at the same time. A general election day may also include elections for local officials.

Voting that occurs at a polling place under the supervision of poll workers on election day. See also: early voting. Syn: polling place voting.

Term as used in election contexts to signify a geographical area to which a practical authority has been granted to administer elections for political offices. Areas of jurisdiction apply to local, state, and federal levels. Note: States, counties, cities, towns and townships are all examples of jurisdictions.

Term as used in election contexts to signify a jurisdiction such as city or town or village that has some form of local government for which elections are generally conducted.

Voting variation in which the voter is entitled to allocate a fixed number of votes $(\mathrm{N})$ over a list of $\mathrm{M}$ contest choices or write-ins, with the constraint that at most 1 vote may be allocated to a given contest choice. Note: This usually occurs when multiple seats are concurrently being filled in a governing body such as a city council or school board where candidates run at-large. The voter is not obliged to allocate all $\mathrm{N}$ votes. 1 -of-M is $\mathrm{N}$-of-M voting where $\mathrm{N}=$ 1.

Election held to narrow the field of candidates in non-party-specific contests prior to the general election. Note: A primary election may be comprised of a nonpartisan primary for some contests or measures and a partisan and/or open primary for others.

Primary held in a state where voters do not register as a party member. Note: There are two variations. In one, the voter declares a choice of party ballot to the pollworker and is issued a ballot containing only contests for that party (and nonpartisan contests). In the other, the ballot issued contains all eligible contest from all parties and the voter selects the party of choice, privately, by only 
Overvote:

\section{Partisan primary:}

Polling place:

Post-election canvass:

Precinct:

Primary election:

Provisional ballot:

Reporting unit: selecting candidates in contests of the desired party. Selections in more than one party void the partisan section of the ballot.

Occurs when a voter selects more than one candidate in a 1-of-M contest or more than $\mathrm{N}$ candidates in an N-of-M contest. The vote for that contest is considered an overvote and not counted towards any candidate in that contest (unless approval voting applies for that contest). Note: Usually the rest of a properly marked ballot is counted. Large numbers of overvotes can be indicative of confusing ballot layout or confusing instructions.

Election held to determine which candidate(s) would represent a political party for particular offices in the general election.

Location at which voters cast ballots in-person on vote-capture devices (e.g., DRE) under the supervision of poll workers usually on election day. Syn: polling station or poll. Note: A polling place is typically in 1-to-1 correspondence with a precinct except for combined precincts and vote centers.

A review of all election results and re-tabulation, resulting in the certification of the results. Generally, the canvass is conducted according to established laws and time frames.

An election administration division corresponding to a contiguous geographic area that is the basis for determining the contests and measures on which the voters legally residing in that area are eligible to vote. Syn: a beat, box, polling district, ward. See also: combined precinct, electoral district, split precinct.

Election generally held to determine which candidate(s) will represent a political party for particular offices in the general election and/or to narrow the field of candidates in non-partyspecific contests prior to the general election. In some cases, such as for local and state central committee members for a particular party, certain contests serve as the sole election as opposed to a primary followed by a general election. Note: From the functional viewpoint of the voting system, the defining features of a primary election are the presence of party-specific contests and a requirement to report separate totals for the different political parties.

Ballot cast by a voter whose eligibility to vote is disputed by an election official. Syn: a challenged ballot.

An administrative division that reports votes or to which votes are associated, e.g., state, county, city, precinct, etc. 
Schema:

Split precinct:

Straight party voting:

Tabulator:

Town:

Township:

UOCAVA voter:

Undervote:

Vote-capture device:

Vote center:

Voter turnout:
A file containing definitions of data elements and attributes with rules for usage, e.g., for XML.

Precinct serving voters from two or more administrative divisions, such as election districts, that may require different ballot styles, other than for primaries. Syn: split.

Voting variation in a general election in which a vote in a designated, special contest (in which the choices are political parties) implies votes in accordance with the endorsements of the selected party in all other contests on the ballot in which straight party voting is allowed. Note: There are different tabulation rules for processing paper ballots containing a straight party selection and direct selections of one or more candidates in individual contests that are state specific.

A programmed device that counts votes.

An urban area that has a name, defined boundaries, and local government, and that is generally larger than a village and smaller than a city.

A subdivision of a county in most northeast and Midwest U.S. states, having the status of a unit of local government with varying governmental powers. Syn: civil township.

From the Uniform and Overseas Citizens Assistance in Voting Act (UOCAVA); A U.S. citizen who is an active member of the Uniformed Services and the Merchant Marine, or the commissioned corps of the Public Health Service or the National Oceanic and Atmospheric Administration, their eligible family members, and U.S. citizens residing outside the United States.

Occurs when the voter does not select a candidate in a 1-of-M contest or selects fewer than $\mathrm{N}$ candidates in an $\mathrm{N}$-of-M contest. Note: can indicate a conscious choice of the voter to not vote in the contest. As with overvotes, large numbers of undervotes can be indicative of confusing ballot layout or confusing instructions.

An automated device that is used directly by a voter to cast a ballot. See also: direct record electronic (DRE).

A polling place where voters from multiple precincts may cast their ballots. Syn: a super precinct. See also: combined precinct.

The number of voters who have cast ballots as a percentage of the total number of voters who can cast ballots. Note: Various states 
Voting variation:

Write-in: calculate this differently, sometimes using the total number of potentially eligible voters whether registered or not.

A contest option or feature, e.g., approval voting, cumulative voting.

Vote for a candidate who is explicitly named by the voter in lieu of choosing a candidate who is already listed on the ballot. Note: In some states, this does not preclude writing in the name of a candidate who is already listed on the ballot. Some states require write-in candidates to be registered prior to the elections to be considered a valid write-in selection. 


\section{Appendix C-References}

W3C, Extensible Markup Language (XML) 1.0 (Fifth Edition), W3C Recommendation, November 26, 2008, http://www.w3.org/TR/xml/ [accessed 2/1/2016].

[4] Florida Department of State, Division of Election, Florida Election Results Summary XML Schema Reference, Version 3.0-S, July 2015, http://dos.myflorida.com/media/695297/ds-de-137-summary.pdf [accessed 2/1/2016].

[5] Voting Information Project (VIP) [Web site], http://votinginfoproject.github.io/vip-specification/ [accessed 2/1/2016].

[6] OASIS, Election Markup Language (EML) Specification Version 7.0, Committee Specification 01, October 27, 2011. Available at: https://www.oasisopen.org/committees/tc_home.php?wg_abbrev=election [accessed 2/1/2016].

[7] City of Cambridge, Massachusetts, Geographic Information Systems: Map Gallery [Web site], https://www.cambridgema.gov/GIS/mapgallery [accessed $2 / 1 / 2016]$.

[8] Hilkert, Scott, “Application of Lessons Learned in the Illinois EDC Project Toward Emerging Election Data Standards and System Guidelines,” submitted to Workshop for a Common Data Format for Electronic Voting Systems, National Institute of Standards and Technology, Gaithersburg, Maryland, October 29-30, 2009, http:/grouper.ieee.org/groups/1622/WorkingDocuments/workshop-2009-10NIST/hilkert-Lessons-Learned-in-Illinois-EDC-1-1.pdf [accessed 2/1/2016]. Recommendation, June 10, 2008, http://www.w3.org/TR/xmldsig-core/ [accessed 2/1/2016].

[10] United States Census Bureau, American National Standards Institute (ANSI) Codes [Web page], https://www.census.gov/geo/reference/ansi.html [accessed 2/1/2016]. 
[11] Open Civic Data, OCD Identifiers [Web page], http://opencivicdata.readthedocs.org/en/latest/ocdids.html [accessed 2/1/2016].

[12] Wikipedia, ISO 639 [Web page], https://en.wikipedia.org/wiki/ISO_639 [accessed 2/1/2016]. 


\section{Appendix D-File Download Locations}

The files associated with this specification are available for download from a NIST repository. These files are:

- This specification,

- XML schema,

- Example XML files,

- Validation tools, and

- UML model.

Other files or updates to the files may be added. The repository URL is located at:

http://vote.nist.gov 


\section{Appendix E-XML Schema}

$<$ ?xml version="1.0" encoding="UTF-8"?>

$<$ !-- Version 1.0-50, January 11, 2016, NIST Election Results CDF Specification -->

$<$ xsd:schema xmlns="NIST_V1_election_results_cdf.xsd" xmlns:ds="http://www.w3.org/2000/09/xmldsig\#"

xmlns:xsd="http://www.w3.org/2001/XMLSchema" targetNamespace="NIST_V1_election_results_cdf.xsd"

elementFormDefault="qualified" version="1.0">

$<!--========$ Imports $=========-->$

<xsd:import namespace="http://www.w3.org/2000/09/xmldsig\#" schemaLocation="http://www.w3.org/2000/09/xmldsig\#"/>

$<!--========$ Roots $=========-->$

$<$ xsd:element name="ElectionReport" type="ElectionReport" $/>$

$<!--========$ Primitives $=========-->$

$<$ xsd:simpleType name $=$ "HtmlColorString" $>$

$<$ xsd:restriction base $=$ "xsd:string" $>$

$<$ xsd:pattern value $="[0-9 \mathrm{a}-\mathrm{f}]\{6\} " />$

$</$ xsd:restriction $>$

$</$ xsd:simpleType $>$

$<$ xsd:simpleType name $=$ "ShortString" $>$

$<$ xsd:restriction base $=$ "xsd:string" >

$<$ xsd:maxLength value $=" 16 " />$

$</$ xsd:restriction $>$

$</$ xsd:simpleType $>$

$<$ xsd:simpleType name="TimeWithZone">

$<$ xsd:restriction base $=$ "xsd:time" $>$

$<$ xsd:pattern value $="(([01][0-9] \mid 2[0-3]):[0-5][0-9]:[0-5][0-9] \mid(24: 00: 00))(Z \mid[+-]((0[0-9] \mid 1[0-3]):[0-5][0-9] \mid 14: 00)) " />$

$<$ xsd:restriction $>$

$</$ xsd:simpleType $>$

$<!--=========$ Enumerations $==========-->$

$<$ xsd:simpleType name="BallotMeasureType">

$<$ xsd:restriction base $=$ "xsd:string" $>$

$<$ xsd:enumeration value $=$ "ballot-measure" $/>$

$<$ xsd:enumeration value $=$ "initiative" $/>$

$<$ xsd:enumeration value $=$ "referendum" $/>$

$<$ xsd:enumeration value $=$ "other" $/>$

$</$ xsd:restriction $>$

$</$ xsd:simpleType $>$

$<$ xsd:simpleType name="CandidatePostElectionStatus" >

$<$ xsd:restriction base $=$ "xsd:string" $>$

$<$ xsd:enumeration value ="advanced-to-runoff" $/>$

$<$ xsd:enumeration value $=$ "projected-winner" $/>$

$<$ xsd:enumeration value $=$ "winner" $/>$

$<$ xsd:enumeration value $=$ "withdrawn" $/>$

$</$ xsd:restriction $>$

$</$ xsd:simpleType $>$

$<$ xsd:simpleType name $=$ "CandidatePreElectionStatus" $>$

$<$ xsd:restriction base $=$ "xsd:string" $>$

$<$ xsd:enumeration value $=$ "filed" $/>$

$<$ xsd:enumeration value $=$ "qualified" $/>$

$<$ xsd:enumeration value $=$ "withdrawn" $/>$

$<$ xsd:enumeration value $=$ "write-in" $/>$

$</$ xsd:restriction $>$

$</$ xsd:simpleType $>$

$<$ xsd:simpleType name="CountItemStatus" $>$

$<$ xsd:restriction base $=$ "xsd:string" $>$

$<$ xsd:enumeration value $=$ "completed" $/>$

$<$ xsd:enumeration value $=$ "in-process" $/>$

$<$ xsd:enumeration value $=$ "not-processed" $/>$

$<$ xsd:enumeration value $=$ "unknown" $/>$

$</$ xsd:restriction $>$

$</$ xsd:simpleType $>$

$<$ xsd:simpleType name="CountItemType" $>$

$<$ xsd:restriction base $=$ "xsd:string" $>$

$<$ xsd:enumeration value $=$ "absentee" $/>$

$<$ xsd:enumeration value $=$ "absentee-fwab" $/>$

$<$ xsd:enumeration value $=$ "absentee-in-person" $/>$

$<$ xsd:enumeration value $=$ absentee-mail" $/>$ 


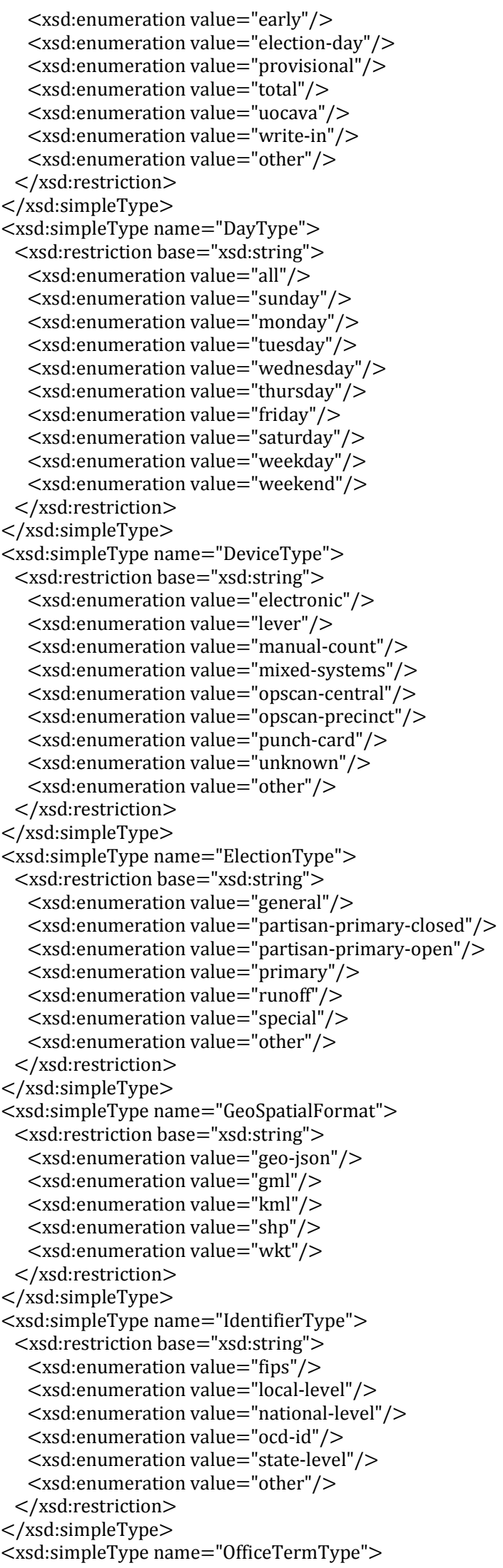




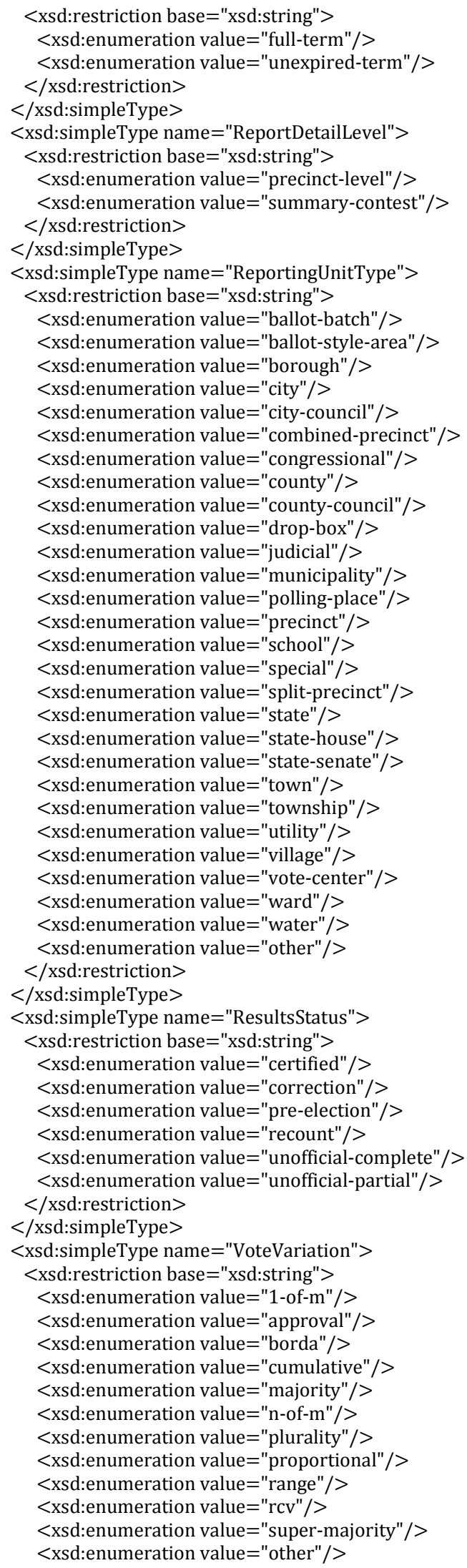




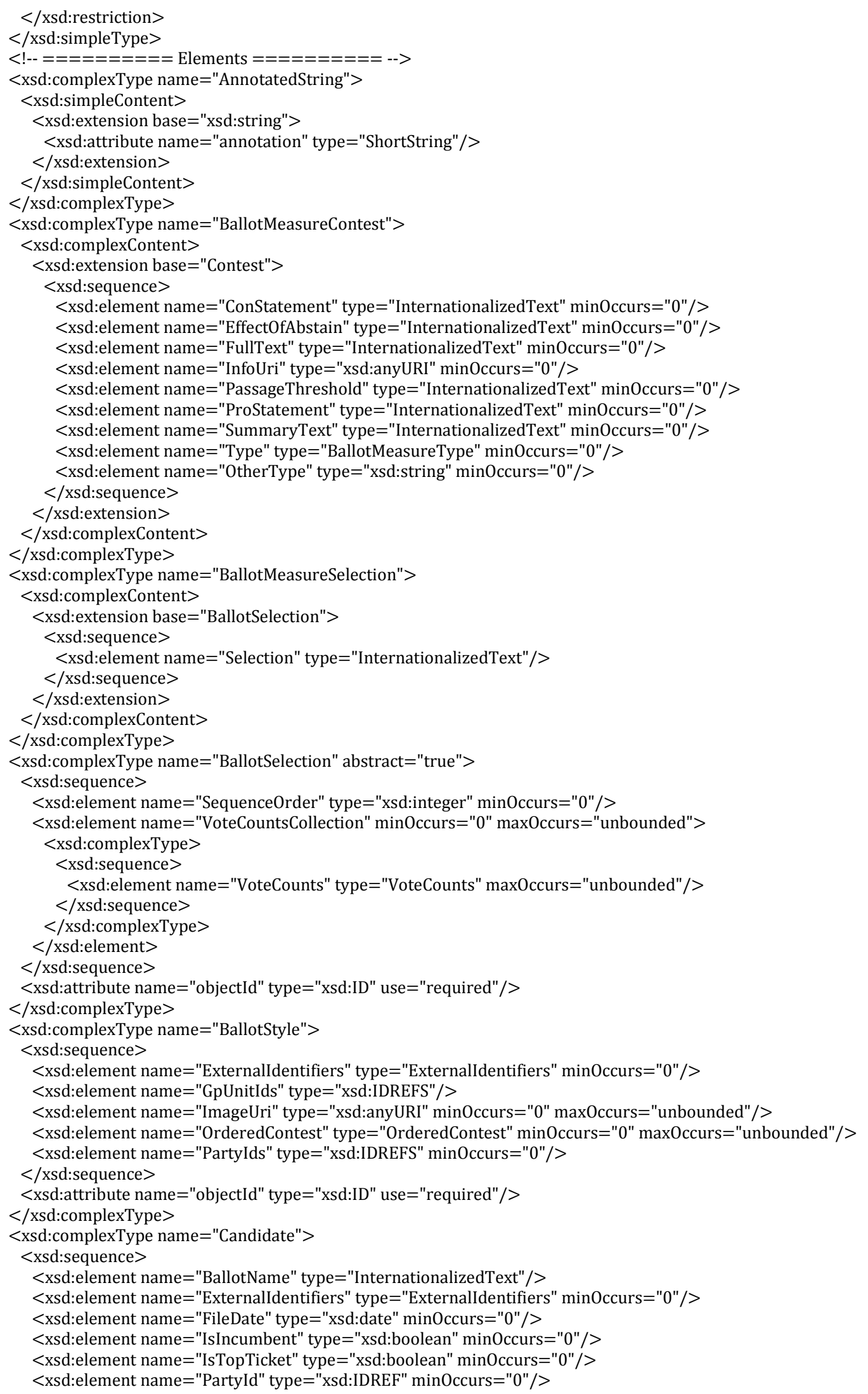




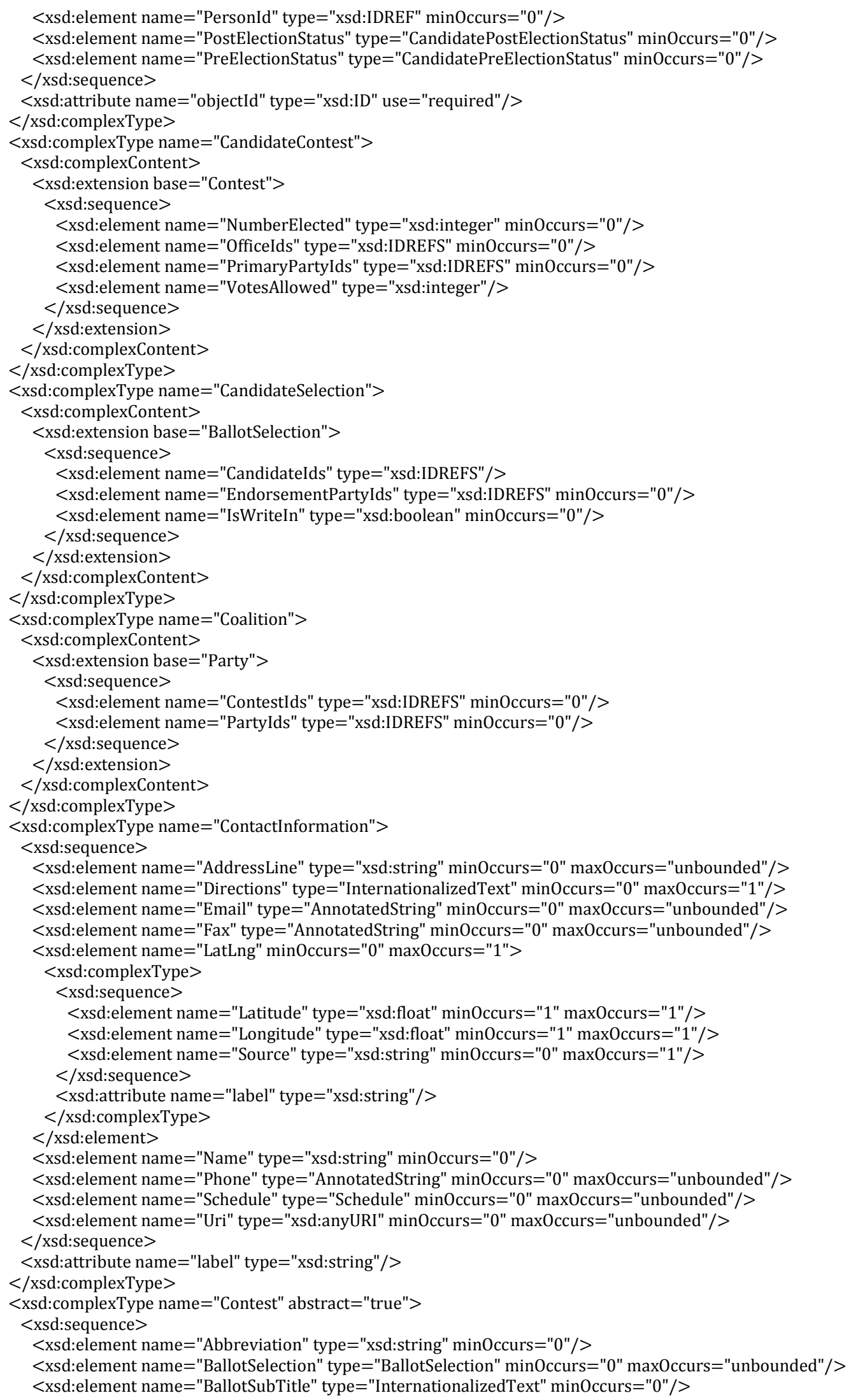


$<$ xsd:element name $=$ "BallotTitle" type $=$ "InternationalizedText" minOccurs $=" 0 " />$

$<$ xsd:element name $=$ "CountStatus" type $=$ "CountStatus" minOccurs $=" 0 "$ maxOccurs $="$ unbounded" $/>$

$<$ xsd:element name="ElectoralDistrictId" type="xsd:IDREF" />

$<$ xsd:element name="ExternalIdentifiers" type="ExternalIdentifiers" minOccurs $=" 0 " />$

$<$ xsd:element name $=$ "HasRotation" type $=$ "xsd:boolean" minOccurs $=" 0 " />$

$<$ xsd:element name="Name" type="xsd:string"/>

$<$ xsd:element name="SequenceOrder" type="xsd:integer" minOccurs="0" $/>$

$<$ xsd:element name="SubUnitsReported" type="xsd:integer" minOccurs="0" />

$<$ xsd:element name $=$ "SummaryCounts" type="SummaryCounts" minOccurs="0" maxOccurs="unbounded" $/>$

$<$ xsd:element name $=$ "TotalSubUnits" type $=$ "xsd:integer" minOccurs $=" 0 " />$

$<$ xsd:element name $=$ "VoteVariation" type $="$ VoteVariation" minOccurs $=" 0 " />$

$<$ xsd:element name $=$ "OtherVoteVariation" type="xsd:string" minOccurs="0" $>$

$</$ xsd:sequence $>$

$<$ xsd:attribute name="objectId" type="xsd:ID" use="required" $/>$

$</$ xsd:complexType $>$

$<$ xsd:complexType name $=$ "CountStatus" $>$

$<$ xsd:sequence $>$

$<$ xsd:element name $=$ "Status" type $=$ "CountItemStatus" $/>$

$<$ xsd:element name $=$ "Type" type $=$ "CountItemType" $/>$

$<$ xsd:element name="OtherType" type="xsd:string" minOccurs $=" 0 " />$

$</$ xsd:sequence $>$

$</$ xsd:complexType $>$

$<$ xsd:complexType name="Counts" abstract="true">

$<$ xsd:sequence $>$

$<$ xsd:element name $="$ Device" type $="$ Device" $\operatorname{minOccurs}=" 0 " />$

$<$ xsd:element name="GpUnitId" type $=$ "xsd:IDREF" minOccurs $=" 0 " />$

$<$ xsd:element name="IsSuppressedForPrivacy" type="xsd:boolean" minOccurs="0" />

$<$ xsd:element name="Type" type $=$ "CountItemType" minOccurs $=" 0 " />$

$<$ xsd:element name $=$ "OtherType" type $=$ "xsd:string" minOccurs $=" 0 " />$

$</$ xsd:sequence $>$

$</$ xsd:complexType $>$

$<$ xsd:complexType name $=$ "Device" $>$

$<$ xsd:sequence $>$

$<$ xsd:element name="Manufacturer" type $=$ "xsd:string" minOccurs $=" 0 " />$

$<$ xsd:element name $=$ "Model" type $=$ "xsd:string" minOccurs $=" 0 " />$

$<$ xsd:element name $=$ "Type" type $=$ "DeviceType" minOccurs $=" 0 " />$

$<$ xsd:element name $=$ "OtherType" type $=$ "xsd:string" minOccurs $=" 0 " />$

$</$ xsd:sequence $>$

$</$ xsd:complexType $>$

$<$ xsd:complexType name="Election" $>$

$<$ xsd:sequence $>$

$<$ xsd:element name="BallotStyleCollection" minOccurs $=" 0 "$ maxOccurs="1">

$<$ xsd:complexType $>$

$<$ xsd:sequence $>$

$<$ xsd:element name="BallotStyle" type="BallotStyle" maxOccurs="unbounded" $/>$

$</$ xsd:sequence $>$

$</$ xsd:complexType $>$

$</$ xsd:element $>$

$<$ xsd:element name="CandidateCollection" minOccurs="0" maxOccurs="1">

$<$ xsd:complexType $>$

$<$ xsd:sequence $>$

$<$ xsd:element name $=$ "Candidate" type $=$ "Candidate" maxOccurs $=$ "unbounded" $/>$

$</$ xsd:sequence $>$

$</$ xsd:complexType $>$

$</$ xsd:element $>$

$<$ xsd:element name="ContactInformation" type="ContactInformation" minOccurs="0" $>>$

$<$ xsd:element name $=$ "ContestCollection" minOccurs="0" maxOccurs="1">

$<$ xsd:complexType >

$<$ xsd:sequence $>$

$<$ xsd:element name="Contest" type $=$ "Contest" maxOccurs="unbounded" $/>$

$</$ xsd:sequence $>$

$</$ xsd:complexType $>$

$</$ xsd:element $>$

$<$ xsd:element name="CountStatus" type="CountStatus" minOccurs="0" maxOccurs $=$ "unbounded" $/>$

$<$ xsd:element name="ElectionScopeId" type="xsd:IDREF" />

$<x$ xd:element name="ExternalIdentifiers" type="ExternalIdentifiers" minOccurs="0" $/>$

$<$ xsd:element name="Name" type="InternationalizedText" /> 


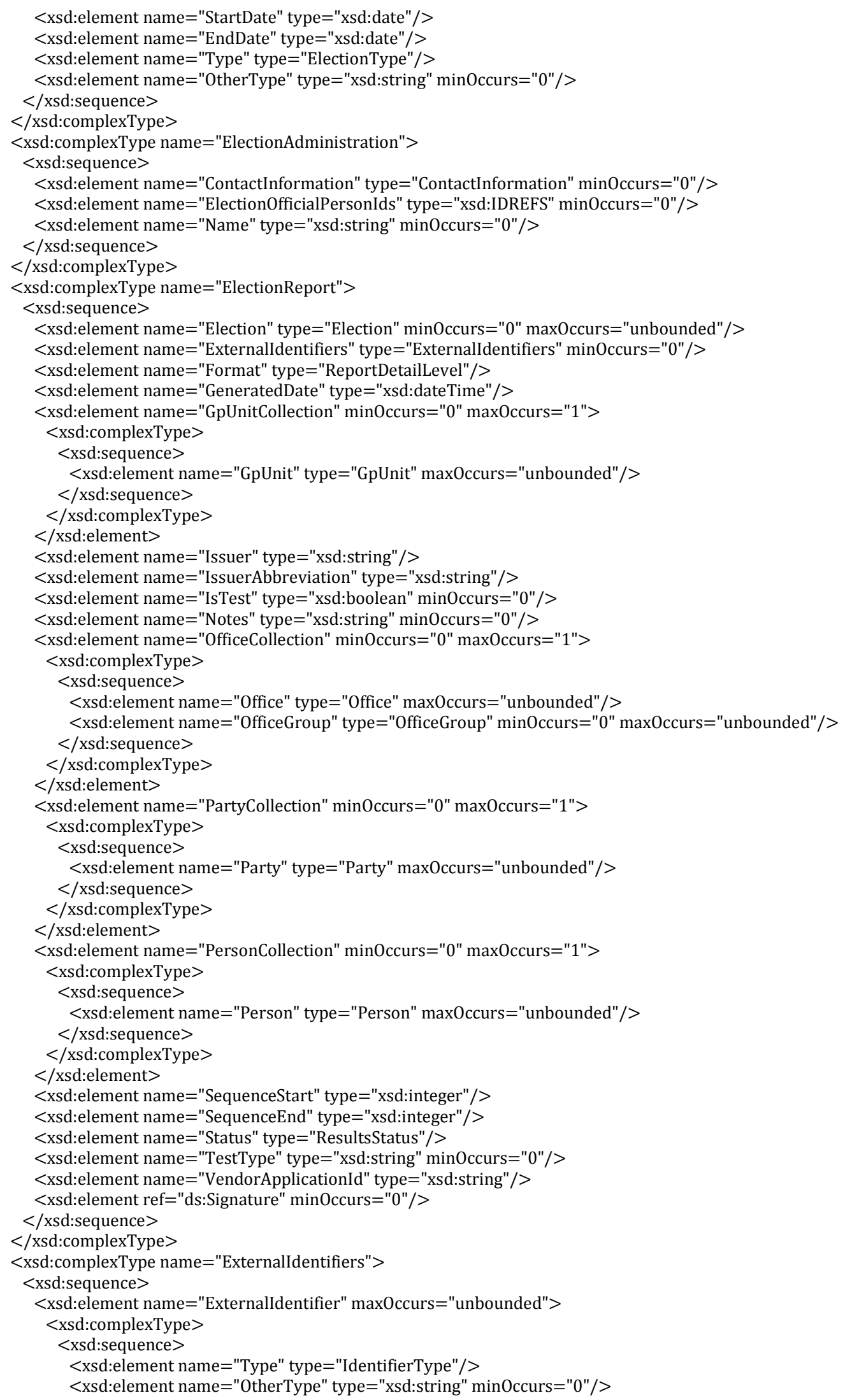




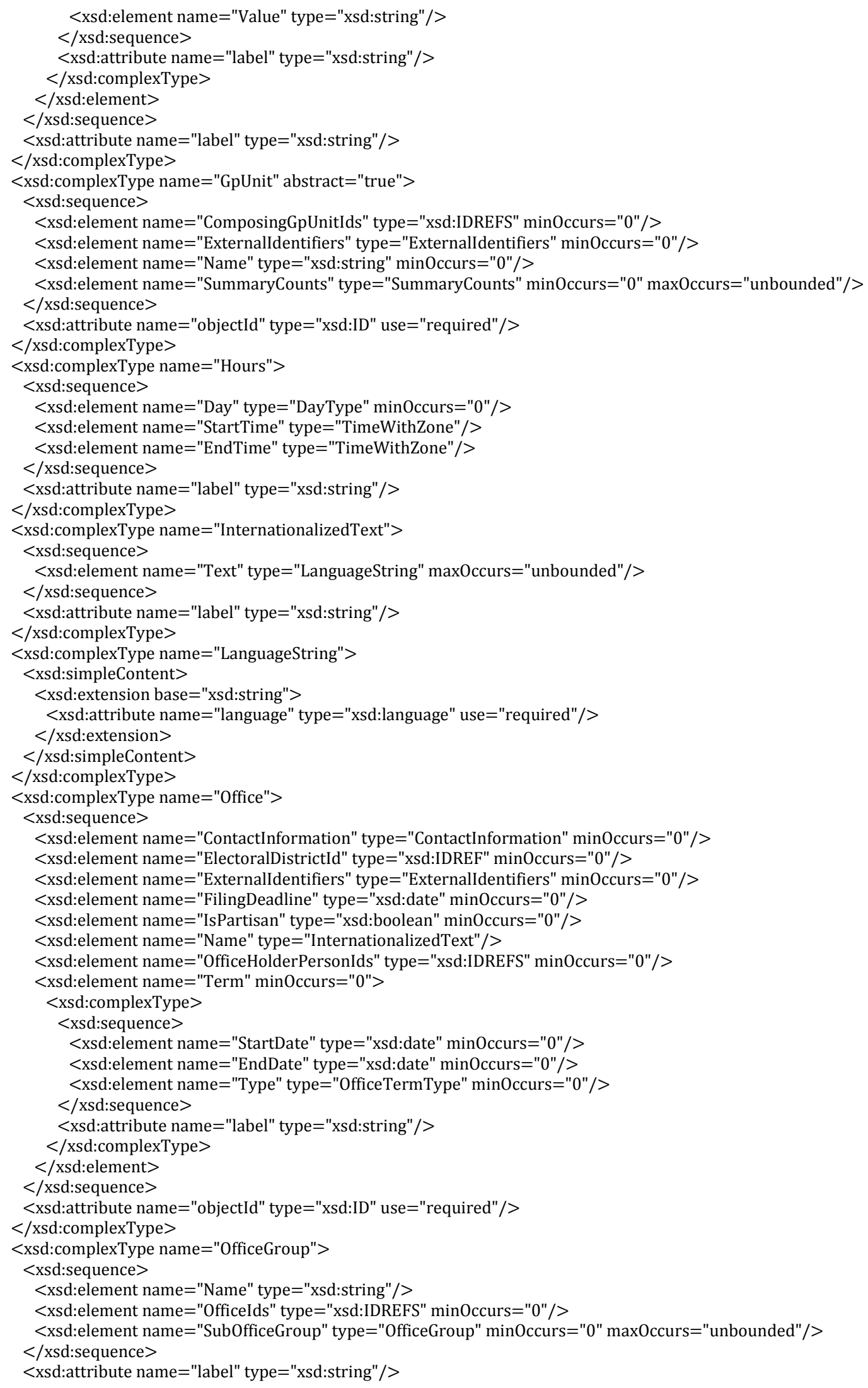




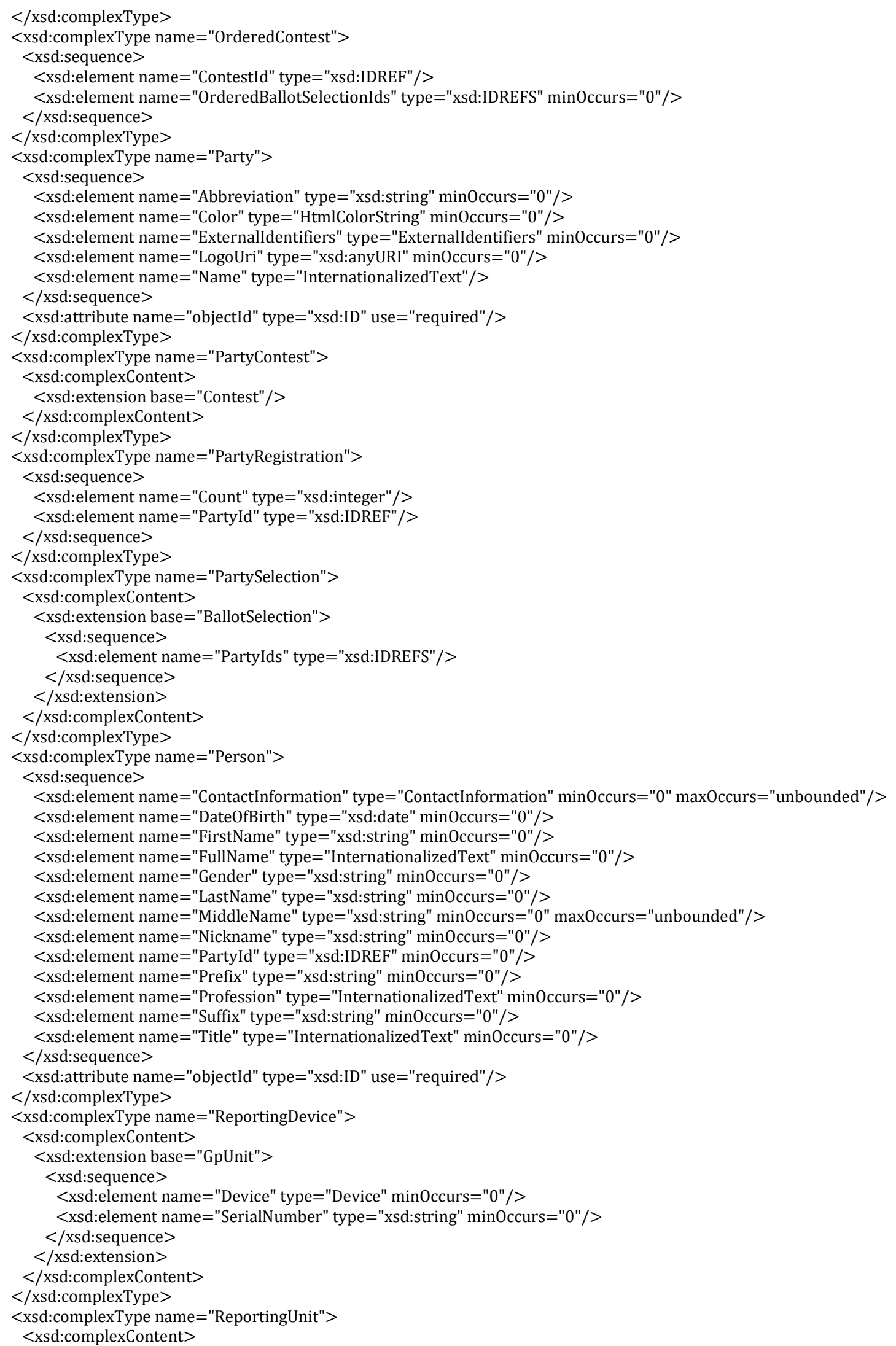




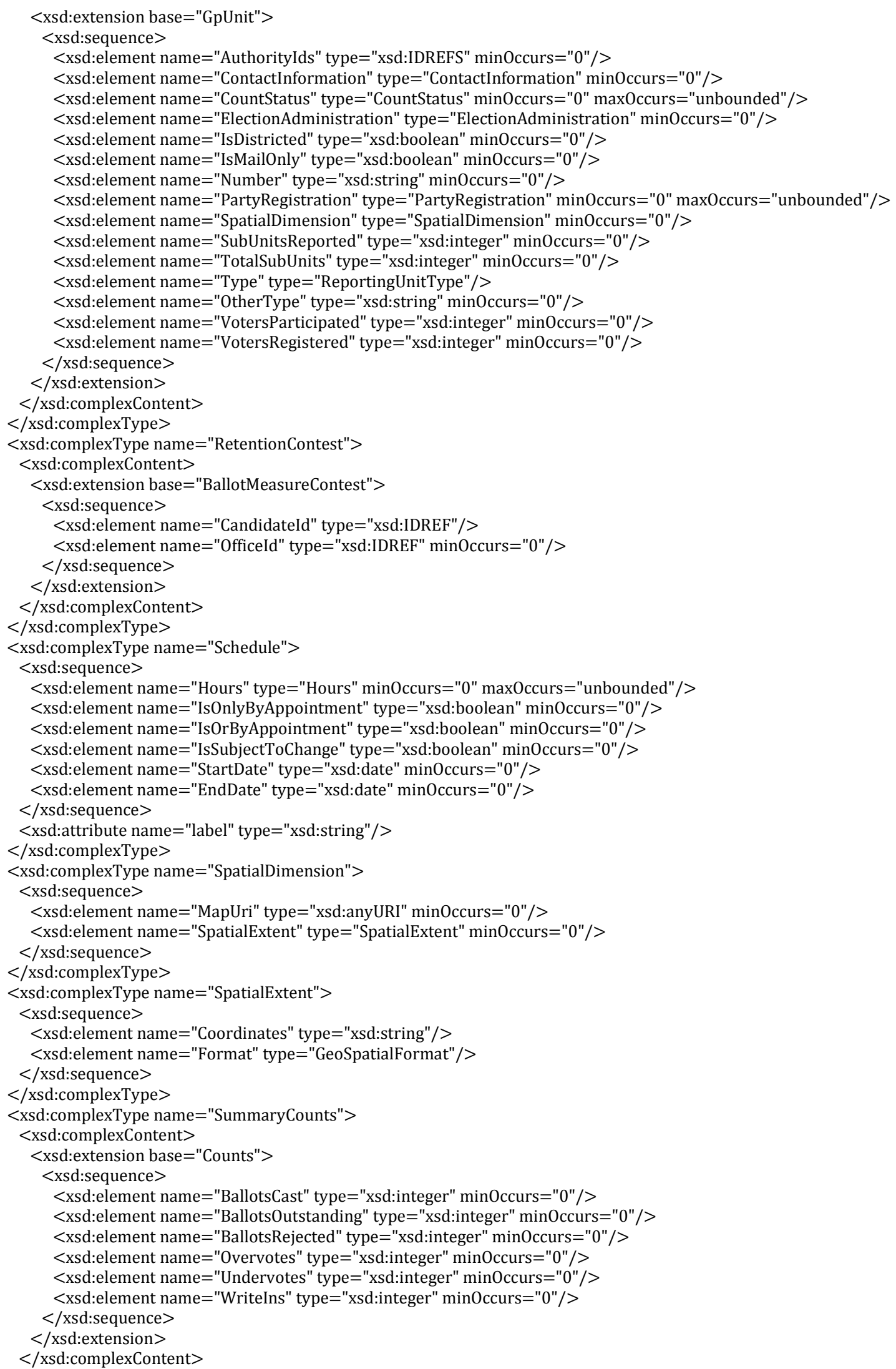




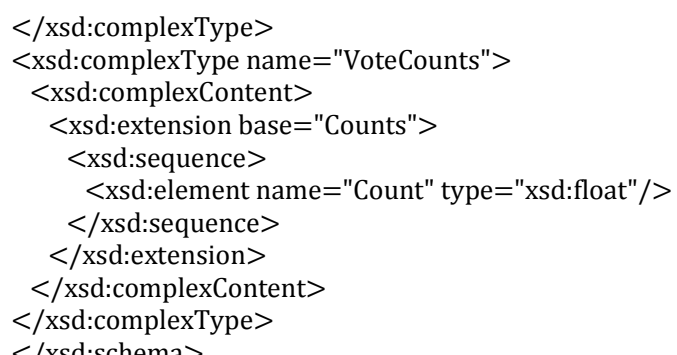

\section{Appendix F-UML Class Diagram - Detailed}

This appendix contains a detailed image of the UML class diagram that when viewed electronically can be expanded to show attributes and other details not shown in the simplified image of the class diagram shown in Figure 8. This image can also be downloaded using the instructions in Appendix D-File Download Locations. 


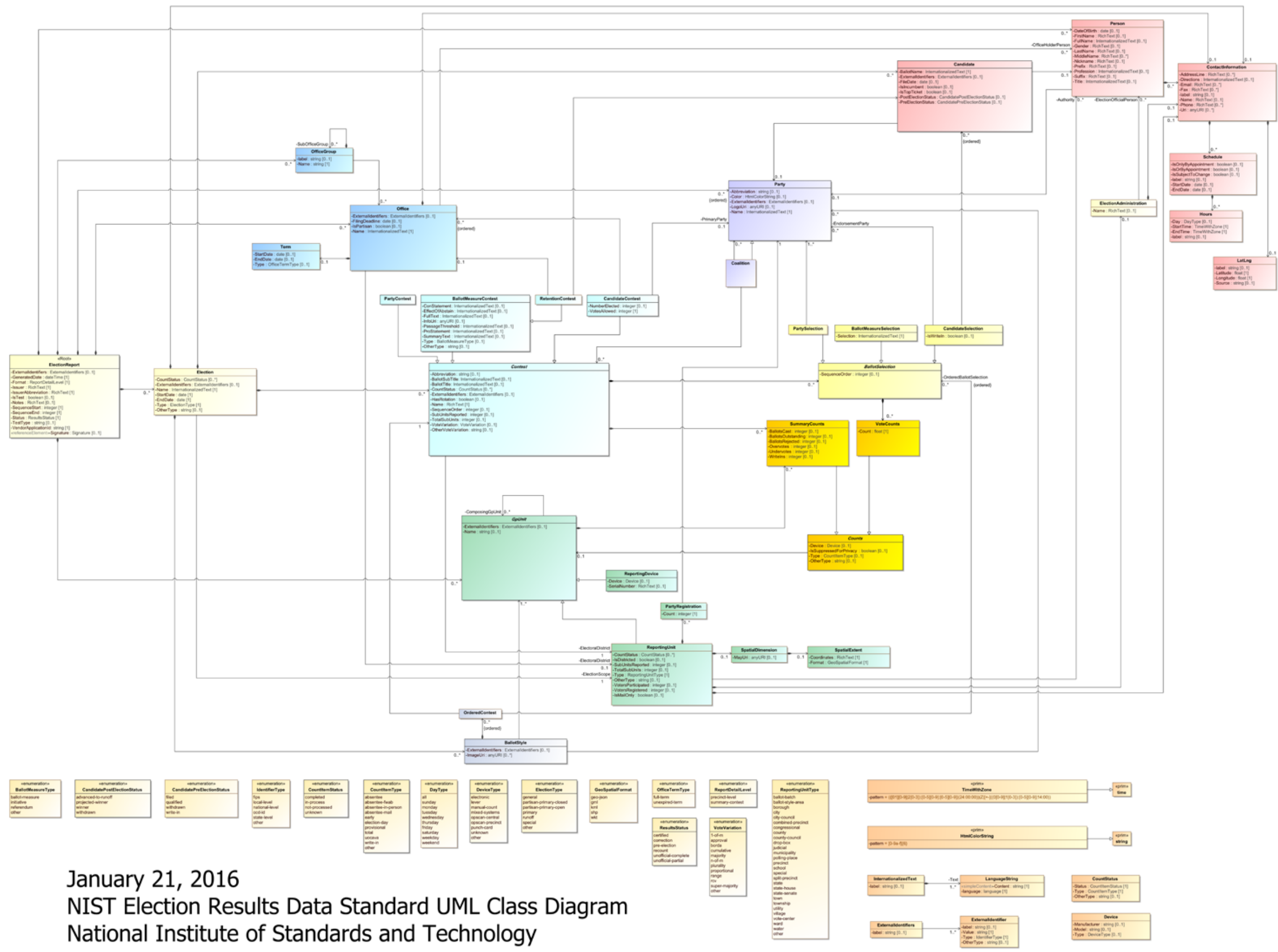

